\title{
Surgery formulae for finite type invariants of rational homology 3-spheres
}

\author{
Christine Lescop
}

We first present four graphic surgery formulae for the degree $n$ part $Z_{n}$ of the Kontsevich-Kuperberg-Thurston universal finite type invariant of rational homology spheres.

Each of these four formulae determines an alternate sum of the form

$$
\sum_{I \subset N}(-1)^{\sharp I} Z_{n}\left(M_{I}\right)
$$

where $N$ is a finite set of disjoint operations to be performed on a rational homology sphere $M$, and $M_{I}$ denotes the manifold resulting from the operations in $I$. The first formula treats the case when $N$ is a set of $2 n$ Lagrangian-preserving surgeries (a Lagrangian-preserving surgery replaces a rational homology handlebody by another such without changing the linking numbers of curves in its exterior). In the second formula, $N$ is a set of $n$ Dehn surgeries on the components of a boundary link. The third formula deals with the case of $3 n$ surgeries on the components of an algebraically split link. The fourth formula is for $2 n$ surgeries on the components of an algebraically split link in which all Milnor triple linking numbers vanish. In the case of homology spheres, these formulae can be seen as a refinement of the Garoufalidis-GoussarovPolyak comparison of different filtrations of the rational vector space freely generated by oriented homology spheres (up to orientation preserving homeomorphisms).

The presented formulae are then applied to the study of the variation of $Z_{n}$ under a $p / q$-surgery on a knot $K$. This variation is a degree $n$ polynomial in $q / p$ when the class of $q / p$ in $\mathbb{Q} / \mathbb{Z}$ is fixed, and the coefficients of these polynomials are knot invariants, for which various topological properties or topological definitions are given.

57M27; 57N10, 57M25, 55R80

\section{Introduction}

In this article, we shall focus on the real finite type invariants of homology 3-spheres in the sense of Ohtsuki, Goussarov and Habiro, and on the topological properties of the surgery formulae that these invariants satisfy. 
M Kontsevich [21] proposed a topological construction for an invariant $Z$ of oriented rational homology 3-spheres using configuration space integrals. G Kuperberg and D Thurston [22] proved that $Z$ is a universal finite type invariant for homology 3spheres, in the sense of Ohtsuki, Goussarov and Habiro; see also Lescop [25]. Like the LMO invariant, the Kontsevich-Kuperberg-Thurston invariant $Z=\left(Z_{n}\right)_{n \in \mathbb{N}}$ takes its values in a space of Jacobi diagrams $\mathcal{A}=\prod_{n \in \mathbb{N}} \mathcal{A}_{n}$, and any real degree $n$ invariant $v$ of homology 3-spheres is obtained from the Kontsevich-Kuperberg-Thurston invariant $\left(Z_{i}\right)_{i \in \mathbb{N}}$ by a composition with a linear form that kills the $Z_{i}$, for $i>n$.

We shall first prove four formulae for $Z_{n}$, for $n \in \mathbb{N}$. Each of these four formulae will determine an alternate sum of the form

$$
\sum_{I \subset N}(-1)^{\sharp I} Z_{n}\left(M_{I}\right)
$$

where $N$ is a set of disjoint operations to be performed on $M$, and $M_{I}$ denotes the manifold resulting from the operations in $I$. Our first formula, Theorem 3.1, will be a mere alternative statement of the main theorem of the author's paper [26] and will treat the case when $N$ is a set of $2 n$ Lagrangian-preserving surgeries (a Lagrangianpreserving surgery replaces a rational homology handlebody by another such without changing the linking numbers of curves in its exterior). In our second formula, Theorem 4.1, $N$ will be a set of $n$ rational surgeries on the components of a boundary link. Our third formula, Theorem 4.3, will deal with the case of $3 n$ surgeries on the components of an algebraically split link. Our fourth formula, Theorem 4.4, will be for $2 n$ surgeries on the components of an algebraically split link in which all Milnor triple linking numbers vanish.

In the case of integral homology spheres, these results can be seen as refinements of the Garoufalidis-Goussarov-Polyak [14] comparison of the filtrations of the vector space generated by homology spheres, with respect to algebraically split links, boundary links or Lagrangian-preserving surgeries; see Auclair and Lescop [3].

As it was proved by Garoufalidis in [13], a degree $n$ finite type invariant $v$ of homology spheres satisfies a surgery formula that describes its variation under $1 / r$-surgery on a knot $K$ as

$$
v(M(K ; 1 / r))-v(M)=\sum_{i=1}^{n} v^{(i)}(K \subset M) r^{i}
$$

where $v^{(i)}$ is a finite type knot invariant in the Vassiliev sense for knots in $S^{3}$ as defined in the Bar-Natan article [6].

Since all the real finite type invariants of homology 3-spheres can be obtained from the universal Le-Murakami-Ohtsuki invariant by composition with a linear form on the 
space of Jacobi diagrams $[23 ; 24]$, and since the LMO invariant is defined using the Kontsevich integral and surgery presentations of manifolds, the knot invariants $v^{(i)}$ can be explicitly given in terms of the Kontsevich integral of surgery presentations of the knots. See also the Århus construction [7; 8; 9] by Bar-Natan, Garoufalidis, Rozansky and Thurston.

We seek a better topological understanding of the invariants $v^{(i)}$, and we shall relate some of them to the topology of Seifert surfaces of the knots. For example, for any degree $n$ invariant $v$, we give a formula in terms of the entries of the Seifert matrix and the weight system of $v$ for the leading coefficient $v^{(n)}$ of the surgery polynomial. See Theorem 5.1. We shall also prove that $v^{(i)}$ is of degree less than $2 n$ for any $i<n$. This specifies a result of Garoufalidis and Habegger [15] who proved that $(v(M(K ; 1))-v(M))$ is a degree $2 n$ knot invariant with the same weight system as a degree $2 n$ knot invariant induced by the Alexander polynomial, by using the LMO invariant.

Some of the results proved in this article can be refined in the extensively studied case of the Casson-Walker invariant $\lambda=W_{1} \circ Z_{1}$, where $W_{1}(\Theta)=2$, that satisfies the well-known formula, for a knot $K$ in a homology sphere $M$,

$$
\lambda(M(K ; p / q))-\lambda(M)=\frac{q}{p} \lambda^{\prime}(K)+\lambda(L(p,-q))
$$

where $\lambda^{\prime}(K)$ is half the second derivative of the normalized Alexander polynomial of $K$ evaluated at 1 and $L(p,-q)$ is the lens space obtained by $p / q$-surgery on the unknot. We shall prove some graphical formulae for $\lambda^{\prime}(K)$ and for its variation under surgeries on disjoint algebraically unlinked knots in Propositions 6.1, 6.2 and 6.3.

Next, we shall concentrate on the case of the degree 2 invariant $\lambda_{2}=W_{2} \circ Z_{2}$, where $W_{2}(\AA)=1$ and $W_{2}(\Theta \Theta)=0$. The invariants $\lambda$ and $\lambda_{2}$ generate the vector space of real-valued invariants of degree lower than 3 that are additive under connected sum. We shall prove that $\lambda_{2}$ satisfies the surgery formula

$$
\lambda_{2}(M(K ; p / q))-\lambda_{2}(M)=\lambda_{2}^{\prime \prime}(K)\left(\frac{q}{p}\right)^{2}+w_{3}(K) \frac{q}{p}+c(q / p) \lambda^{\prime}(K)+\lambda_{2}(L(p,-q))
$$

for a knot $K$ in a homology sphere $M$, where $c(q / p)$ only depends on $q / p$ modulo $\mathbb{Z}$, $\lambda_{2}^{\prime \prime}$ is explicitly given in Theorem 5.1 and $w_{3}$ is a knot invariant, for which we shall prove various properties. These properties include a crossing change formula, Proposition 7.2 , and a formula for genus one knots $K$, Proposition 7.3. For knots in $S^{3}, w_{3}$ is the degree 3 knot invariant that changes sign under mirror image, and that maps the chord diagram with three diameters to $(-1)$. In his thesis [2], Emmanuel Auclair independently obtained a formula for $w_{3}(K)$ in terms of topological invariants of 
curves of an arbitrary Seifert surface of $K$, that is fortunately equivalent to Proposition 7.3 in the genus one case.

The article is organized as follows. The main results are stated precisely without proofs from Section 3 to Section 7. The proofs occupy the following sections. Questions and expected generalizations of the proved results are mentioned at the end.

\section{The Kontsevich-Kuperberg-Thurston universal finite type invariant}

\subsection{Jacobi diagrams}

Here, a Jacobi diagram $\Gamma$ is a trivalent graph $\Gamma$ without simple loop like $-\bigcirc$. The set of vertices of such a $\Gamma$ is denoted by $V(\Gamma)$, its set of edges is denoted by $E(\Gamma)$. A half-edge $c$ of $\Gamma$ is an element of

$$
H(\Gamma)=\{c=(v(c) ; e(c)) \mid v(c) \in V(\Gamma) ; e(c) \in E(\Gamma) ; v(c) \in e(c)\} .
$$

An automorphism of $\Gamma$ is a permutation $b$ of $H(\Gamma)$ such that for any $c, c^{\prime} \in H(\Gamma)$,

$$
v(c)=v\left(c^{\prime}\right) \Longrightarrow v(b(c))=v\left(b\left(c^{\prime}\right)\right) \text { and } e(c)=e\left(c^{\prime}\right) \Longrightarrow e(b(c))=e\left(b\left(c^{\prime}\right)\right)
$$

The number of automorphisms of $\Gamma$ is denoted by $\sharp \operatorname{Aut}(\Gamma)$. For example, $\sharp$ Aut $(\Theta)=$ 12. An orientation of a vertex of such a diagram $\Gamma$ is a cyclic order of the three half-edges that meet at that vertex. A Jacobi diagram $\Gamma$ is oriented if all its vertices are oriented (equipped with an orientation). The degree of such a diagram is half the number of its vertices.

Let $\mathcal{A}_{n}=\mathcal{A}_{n}(\varnothing)$ denote the real vector space generated by the (isomorphism classes of) degree $n$ oriented Jacobi diagrams, quotiented out by the following relations AS and IHX:

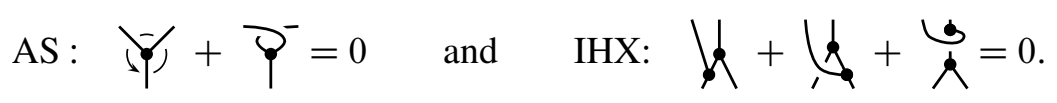

Each of these relations relate diagrams which can be represented by planar immersions that are identical outside the part of them represented in the pictures. Here, the orientation of vertices is induced by the counterclockwise order of the half-edges. For example, AS identifies the sum of two diagrams which only differ by the orientation at one vertex to zero. $\mathcal{A}_{0}(\varnothing)$ is equal to $\mathbb{R}$ generated by the empty diagram. 


\subsection{The Kontsevich-Kuperberg-Thurston universal finite type invariant}

Let $\Lambda$ be $\mathbb{Z}, \mathbb{Z} / 2 \mathbb{Z}$ or $\mathbb{Q}$. A $\Lambda$-sphere is a compact oriented 3-manifold $M$ such that $H_{*}(M ; \Lambda)=H_{*}\left(S^{3} ; \Lambda\right)$. A $\mathbb{Z}$-sphere is also called a homology sphere while a rational homology sphere is a $\mathbb{Q}$-sphere. Following Witten, Axelrod and Singer [4; 5], Kontsevich [21], Bott and Cattaneo [10; 11; 12], Greg Kuperberg and Dylan Thurston constructed invariants $Z_{n}=\left(Z_{K K T}\right)_{n}$ of oriented $\mathbb{Q}$-spheres with values in $\mathcal{A}_{n}(\varnothing)$ and they proved that their invariants have the following property [22]. See also Lescop $[25 ; 26]$.

Theorem 2.1 (Kuperberg-Thurston [22]) An invariant $v$ of $\mathbb{Z}$-spheres with values in a real vector space $X$ is of degree $\leq n$ if and only if there exist linear maps

$$
\phi_{k}(v): \mathcal{A}_{k}(\varnothing) \longrightarrow X
$$

for any $k \leq n$, such that

$$
v=\sum_{k=0}^{n} \phi_{k}(v) \circ Z_{k}
$$

A real finite type invariant of $\mathbb{Z}$-spheres is a topological invariant of $\mathbb{Z}$-spheres with values in a real vector space $X$ which is of degree less than some natural integer $n$. Theorem 2.1 can be used as a definition of degree $\leq n$ real-valued invariants of $\mathbb{Z}$-spheres.

A degree $\leq n$ invariant $v$ is of degree $n$ if $\phi_{n}(v) \neq 0$. In this case, $\phi_{n}(v)$ is the weight system of $v$ and is denoted by $W_{v}$.

Let $p^{c}: \mathcal{A}_{k}(\varnothing) \rightarrow \mathcal{A}_{k}(\varnothing)$ be the canonical linear projection of $\mathcal{A}_{k}(\varnothing)$ onto its subspace $\mathcal{A}_{k}^{c}(\varnothing)$ generated by the connected diagrams, such that $p^{c}$ maps the nonconnected diagrams to 0 and the restriction of $p^{c}$ to $\mathcal{A}_{k}^{c}(\varnothing)$ is of course the identity. Then $Z_{n}^{c}=p^{c} \circ Z_{n}$ is additive under connected sum. Furthermore any real-valued degree $n$ invariant belongs to the algebra generated by the $\left(\phi_{k, i} \circ Z_{k}^{c}\right)_{k \leq n}$ for linear forms $\phi_{k, i}$ generating the dual of $\mathcal{A}_{k}^{c}(\varnothing)$.

Remark 2.2 The above definition coincides with the Ohtsuki definition of real finite type invariants [33]. The Ohtsuki degree (that is always a multiple of 3 ) is three times the above degree. See Auclair and Lescop [3], Garoufalidis, Goussarov and Polyak [14], Habiro [19] and Ohtsuki [33] and references therein for more discussions about the various concepts of finite type invariants. 


\section{Lagrangian-preserving surgeries}

Conventions Unless otherwise stated, manifolds are compact and oriented. Boundaries are always oriented with the outward normal first convention. The normal bundle $N(V)$ of an oriented submanifold $V$ in an oriented manifold $M$ is oriented so that the tangent bundle $T_{x} M$ of the ambient manifold $M$ at some $x \in V$ is oriented as $T_{x} M=N_{x} V \oplus T_{x} V$. If $V$ and $W$ are two oriented transverse submanifolds of an oriented manifold $M$, their intersection is oriented so that the normal bundle of $T_{x}(V \cap W)$ is the sum $N_{x} V \oplus N_{x} W$. If the two manifolds are of complementary dimensions, then the sign of an intersection point is +1 if the orientation of its normal bundle coincides with the orientation of the ambient space that is equivalent to say that $T_{x} M=T_{x} V \oplus T_{x} W$. Otherwise, the sign is -1 . If $V$ and $W$ are compact and if $V$ and $W$ are of complementary dimensions in $M$, their algebraic intersection is the sum of the signs of the intersection points, it is denoted by $\langle V, W\rangle_{M}$.

Recall that the linking number $\operatorname{lk}(J, K)$ of two disjoint knots $J$ and $K$ in a rational homology sphere $M$ is the algebraic intersection of $J$ with a surface $\Sigma_{K}$ bounded by $K$ if $K$ is null-homologous, that $\operatorname{lk}(J,$.$) is linear on H_{1}(M \backslash J)$, and that $\operatorname{lk}(J, K)=$ $\operatorname{lk}(K, J)$.

The Milnor triple linking number $\mu\left(K_{1}, K_{2}, K_{3}\right)$ of three null-homologous knots $K_{1}$, $K_{2}, K_{3}$ that do not link each other algebraically in a rational homology sphere $M$ may be defined, as the algebraic intersection of three Seifert surfaces $\Sigma_{2}, \Sigma_{1}, \Sigma_{3}$ of these knots in the complement of the other ones.

$$
\mu\left(K_{1}, K_{2}, K_{3}\right)=-\left\langle\Sigma_{1}, \Sigma_{2}, \Sigma_{3}\right\rangle=-\left\langle\Sigma_{1} \cap \Sigma_{2}, \Sigma_{3}\right\rangle=-\operatorname{lk}\left(K_{3}, \Sigma_{1} \cap \Sigma_{2}\right) .
$$

We now describe part of the behaviour of the $Z_{n}$ under the Lagrangian-preserving surgeries defined below.

A genus $g \mathbb{Q}$-handlebody is an (oriented, compact) 3-manifold $A$ with the same homology with rational coefficients as the standard (solid) handlebody $H_{g}$ of Figure 1. Note that the boundary of such a $\mathbb{Q}$-handlebody $A$ is homeomorphic to the boundary $\left(\partial H_{g}=\Sigma_{g}\right)$ of $H_{g}$.

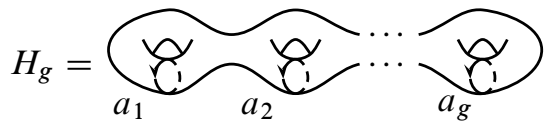

Figure 1: The standard handlebody $H_{g}$

For a $\mathbb{Q}$-handlebody $A, \mathcal{L}_{A}$ denotes the kernel of the map induced by the inclusion

$$
H_{1}(\partial A ; \mathbb{Q}) \longrightarrow H_{1}(A ; \mathbb{Q}) .
$$


It is a Lagrangian of $\left(H_{1}(\partial A ; \mathbb{Q}),\langle,\rangle_{\partial A}\right)$, we call it the Lagrangian of $A$. A Lagrangian-preserving surgery or LP-surgery $\left(A, A^{\prime}\right)$ is the replacement of a $\mathbb{Q}$-handlebody $A$ embedded in a 3-manifold by another such $A^{\prime}$ with identical (identified via a homeomorphism) boundary and Lagrangian.

There is a canonical isomorphism

$$
\partial_{M V}: H_{2}\left(A \cup_{\partial A}-A^{\prime} ; \mathbb{Q}\right) \rightarrow \mathcal{L}_{A}
$$

that maps the class of a closed surface in the closed 3-manifold $\left(A \cup_{\partial A}-A^{\prime}\right)$ to the boundary of its intersection with $A$. This isomorphism carries the algebraic triple intersection of surfaces to a trilinear antisymmetric form $\mathcal{I}_{A A^{\prime}}$ on $\mathcal{L}_{A}$.

$$
\mathcal{I}_{A A^{\prime}}\left(a_{i}, a_{j}, a_{k}\right)=\left\langle\partial_{M V}^{-1}\left(a_{i}\right), \partial_{M V}^{-1}\left(a_{j}\right), \partial_{M V}^{-1}\left(a_{k}\right)\right\rangle_{A \cup-A^{\prime}}
$$

Let $\left(a_{1}, a_{2}, \ldots, a_{g}\right)$ be a basis of $\mathcal{L}_{A}$, and let $z_{1}, \ldots, z_{g}$ be homology classes of $\partial A$, such that $\left(z_{1}, \ldots, z_{g}\right)$ is dual to $\left(a_{1}, a_{2}, \ldots, a_{g}\right)$ with respect to $\langle,\rangle_{\partial A}\left(\left\langle a_{i}, z_{j}\right\rangle_{\partial A}=\right.$ $\left.\delta_{i j}\right)$. Note that $\left(z_{1}, \ldots, z_{g}\right)$ is a basis of $H_{1}(A ; \mathbb{Q})$.

Represent $\mathcal{I}_{A A^{\prime}}$ by the following combination $T\left(\mathcal{I}_{A A^{\prime}}\right)$ of tripods whose three univalent vertices form an ordered set:

$$
T\left(\mathcal{I}_{A A^{\prime}}\right)=\sum_{\left\{\{i, j, k\} \subset\left\{1,2, \ldots, g_{A}\right\} ; i<j<k\right\}} \mathcal{I}_{A A^{\prime}}\left(a_{i}, a_{j}, a_{k}\right)<{ }_{z_{j}}^{z_{k}}
$$

When $G$ is a graph with $2 n$ trivalent vertices and with univalent vertices decorated with disjoint curves of $M$, define its contraction $\left\langle\langle G\rangle_{n}\right.$ as the sum that runs over all the ways $p$ of gluing the univalent vertices two by two in order to produce a vertex-oriented Jacobi diagram $G_{p}$

$$
\langle\langle G\rangle\rangle_{n}=\sum_{p} \ell\left(G_{p}\right)\left[G_{p}\right]
$$

where $\ell\left(G_{p}\right)$ is the product over the pairs of glued univalent vertices, with respect to the pairing $p$, of the linking numbers of the corresponding curves. The contraction $\langle\langle\cdot\rangle\rangle$ is linearly extended to linear combination of graphs, and the disjoint union of combinations of graphs is bilinear.

A $k$-component Lagrangian-preserving surgery datum in a rational homology sphere $M$ is a datum $\left(M ;\left(A_{i}, A_{i}^{\prime}\right)_{i \in\{1, \ldots, k\}}\right)$ of $k$ disjoint $\mathbb{Q}$-handlebodies $A_{i}$, for $i \in$ $\{1, \ldots, k\}$, in $M$, and $k$ associated LP-surgeries $\left(A_{i}, A_{i}^{\prime}\right)$. 


\section{Theorem 3.1 Let}

$$
\left(M ;\left(A_{i}, A_{i}^{\prime}\right)_{i \in\{1, \ldots, 2 n\}}\right)
$$

be a $2 n$-component Lagrangian-preserving surgery datum in a rational homology sphere $M$. For $I \subset\{1, \ldots, 2 n\}$, let $M_{I}$ denote the manifold obtained from $M$ by replacing $A_{i}$ by $A_{i}^{\prime}$ for all $i \in I$. Then

$$
\sum_{I \subset\{1, \ldots, 2 n\}}(-1)^{\sharp I} Z_{n}\left(M_{I}\right)=\left\langle\bigsqcup_{i \in\{1, \ldots, 2 n\}} T\left(\mathcal{I}_{A_{i} A_{i}^{\prime}}\right) \|_{n} .\right.
$$

We shall prove that this formula is equivalent to the author's formula of [26] in Section 8.

Let $\mathcal{F}_{0}$ be the rational vector space freely generated by the oriented $\mathbb{Q}$-spheres viewed up to oriented homeomorphisms. For a $k$-component Lagrangian-preserving surgery datum $\left(M ;\left(A_{i}, A_{i}^{\prime}\right)_{i \in\{1, \ldots, k\}}\right)$ in a rational homology sphere $M$, define

$$
\left[M ;\left(A_{i}, A_{i}^{\prime}\right)_{i \in\{1, \ldots, k\}}\right]=\sum_{I \subset\{1, \ldots, k\}}(-1)^{\sharp I} M_{I} \in \mathcal{F}_{0}
$$

and define $\mathcal{F}_{k}$ as the subspace of $\mathcal{F}_{0}$ generated by elements of $\mathcal{F}_{0}$ of the above form. Then, it easily follows from the above theorem that $Z_{n}\left(\mathcal{F}_{2 n+1}\right)=0$ where $Z_{n}$ is linearly extended to $\mathcal{F}_{0}$. For two elements $x$ and $y$ of $\mathcal{F}_{0}$, we write $x \equiv_{n} y$ to say that $x-y \in \mathcal{F}_{2 n+1}$. Thus, if $x \equiv_{n} y$, then $Z_{n}(x)=Z_{n}(y)$.

The intersection of this filtration with the rational vector space freely generated by the oriented $\mathbb{Z}$-spheres is the Goussarov-Habiro filtration. (The inclusion of the Goussarov-Habiro filtration $\left(\mathcal{F}_{k}^{\mathrm{GH}}\right)_{k}$ in the intersection is obvious, the other one comes from the fact that $\mathcal{F}_{k}^{\mathrm{GH}}$ is the intersection of the kernels of the $Z_{i}$ for $2 i<k$ because of the universality of $Z$.)

\section{Surgeries on algebraically split links}

Let $L\left(p_{i},-q_{i}\right)$ be the lens space obtained from $S^{3}$ by $p_{i} / q_{i}$-surgery on a trivial knot. (The standard conventions for surgery coefficients are recalled in the beginning of Section 6.) When $L=\left(K_{i} ; p_{i} / q_{i}\right)_{i \in N}$ is a given link whose components are equipped with surgery coefficients in a rational homology sphere $M$, for $I \subset N$, let

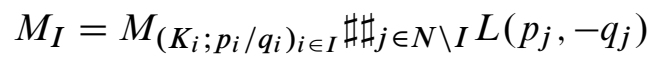

denote the connected sum of the manifold $M_{\left(K_{i} ; p_{i} / q_{i}\right)_{i \in I}}=M\left(\left(K_{i} ; p_{i} / q_{i}\right)_{i \in I}\right)$ obtained from $M$ by surgery on $\left(K_{i} ; p_{i} / q_{i}\right)_{i \in I}$ and the lens spaces $L\left(p_{j},-q_{j}\right)$ for $j \notin I$. 
Set

$$
\left[M ;\left(K_{i} ; p_{i} / q_{i}\right)_{i \in N}\right]=\sum_{I \subset N}(-1)^{\sharp I} M_{I} .
$$

Note that the connected sums with lens spaces are trivial when the $p_{i}$ are 1.

The invariant $Z_{n}$ is linearly extended to $\mathcal{F}_{0}$. By the additivity of the connected part $Z_{n}^{c}$ of $Z_{n}$ under connected sum, if $N$ has more than one element,

$$
Z_{n}^{c}\left(\left[M ;\left(K_{i} ; p_{i} / q_{i}\right)_{i \in N}\right]\right)=Z_{n}^{c}\left(\sum_{I \subset N}(-1)^{\sharp I} M_{\left(K_{i} ; p_{i} / q_{i}\right)_{i \in I}}\right)
$$

and the connected sums with lens spaces do not appear in this case either.

In Section 9, we shall see how Theorem 3.1 easily implies the following surgery formula on $n$-component boundary links.

Theorem 4.1 Let $n$ and $r$ be elements of $\mathbb{N}$. Consider a link $\left(K_{1}, K_{2}, \ldots, K_{r}\right)$ where all the $K_{i}$ bound disjoint oriented surfaces $\Sigma^{i}$. Let $p_{i} / q_{i}$ be a surgery coefficient for $K_{i}$, and let $\left(x_{j}^{i}, y_{j}^{i}\right)_{j=1, \ldots, g\left(\Sigma^{i}\right)}$ be a symplectic basis for the Seifert surface $\Sigma^{i}$. Define

$$
I\left(\Sigma^{i}\right)=\sum_{(j, k) \in\left\{1,2, \ldots, g\left(\Sigma^{i}\right)\right\}^{2}} x_{j}^{i} y_{j}^{i}\left(y_{k}^{i}\right)^{+}\left(x_{k}^{i}\right)^{+} .
$$

Then

$$
Z_{n}\left(\left[M ;\left(K_{i} ; p_{i} / q_{i}\right)_{i \in\{1, \ldots, r\}}\right]\right)= \begin{cases}0 & \text { if } r>n \\ \frac{1}{2^{n}}\left\langle\left\langle\bigsqcup_{i \in\{1, \ldots, n\}}\left(-\frac{q_{i}}{p_{i}} I\left(\Sigma^{i}\right)\right)\right\rangle\right\rangle & \text { if } r=n .\end{cases}
$$

Definition 4.2 A link $L$ in a 3-manifold is said to be algebraically split if any component of $L$ is null-homologous in the exterior of the other ones (ie if any component of $L$ bounds a surface in the complement of the other components of $L$ ).

An edge-labelled Jacobi diagram is a Jacobi diagram $\Gamma$ equipped with a bijection from $E(\Gamma)$ to $\{1,2,3, \ldots, 3 n\}$ for some integer $n$. Let $D_{e, n}$ be the set of (isomorphism classes of) unoriented edge-labelled Jacobi diagrams of degree $n$. Let $L=\left(K_{i}\right)_{i \in\{1,2,3, \ldots, 3 n\}}$ be a $3 n$-component algebraically split link. Let $\Gamma \in D_{e, n}$, orient $\Gamma$. With any vertex of $\Gamma$, whose incoming edges are labeled by $i, j, k$ with respect to the cyclic order induced by the orientation, associate the Milnor triple number $\mu\left(K_{i}, K_{j}, K_{k}\right)$. Then define $\mu_{\Gamma}(L)$ as the product over all the vertices of $\Gamma$ of the corresponding Milnor numbers of $L$. Note that $\mu_{\Gamma}(L)[\Gamma]$ does not depend on the orientation of $\Gamma$. Let $\theta(\Gamma)$ be the number of components of $\Gamma$ homeomorphic to $\Theta$. 
Theorem 4.3 Let $n$ and $r$ be elements of $\mathbb{N}$. Let $L=\left(K_{i} ; p_{i} / q_{i}\right)_{i \in\{1,2,3, \ldots, r\}}$ be a (rationally) framed $r$-component algebraically split link in a rational homology sphere $M$. Then with the notation above,

$$
Z_{n}\left(\left[M ;\left(K_{i} ; p_{i} / q_{i}\right)_{i \in\{1, \ldots, r\}}\right]\right)= \begin{cases}0 & \text { if } r>3 n \\ \left(\prod_{i=1}^{3 n} \frac{q_{i}}{p_{i}}\right) \sum_{\Gamma \in D_{e, n}} \frac{\mu_{\Gamma}(L)}{2^{\theta(\Gamma)}}[\Gamma] & \text { if } r=3 n\end{cases}
$$

A 2/3-labelled Jacobi diagram is a degree $n$ Jacobi diagram $\Gamma$ equipped with an injection $\iota$ from $\{1,2,3, \ldots, 2 n\}$ to $E(\Gamma)$ such that, at each vertex, two edges of the image of $\iota$ meet one edge outside the image of $\iota$. Let $D_{2 / 3, n}$ be the set of (isomorphism classes of) unoriented 2/3-labelled Jacobi diagrams of degree $n$. Let $\left(F_{i}\right)_{i=1, \ldots 2 n}$ be a collection of transverse oriented surfaces that meet pairwise only inside their respective interiors, such that $\left\langle F_{i}, F_{j}, F_{k}\right\rangle=0$ for any $\{i, j, k\} \subset\{1,2,3, \ldots, 2 n\}$. Let $\Gamma \in D_{2 / 3, n}$, orient $\Gamma$. For a vertex of $\Gamma$, whose half-edges belong to edges labelled by $(i, j$, nothing), with respect to the cyclic order induced by the orientation, assign the intersection curve $F_{i} \cap F_{j}$ to the unlabelled half-edge. To any unlabelled edge $e$ that is now equipped with intersection curves $F_{i} \cap F_{j}$ and $F_{k} \cap F_{\ell}$ associate the linking number $\ell\left(\left(F_{i}\right)_{i=1, \ldots 2 n} ; \Gamma ; e\right)$ of $F_{i} \cap F_{j}$ and $F_{k}^{+} \cap F_{\ell}^{+}$, where $F_{k}^{+}$and $F_{\ell}^{+}$ are parallel copies of $F_{k}$ and $F_{\ell}$.

Note that there is no need to push the intersection curves by using parallels if $F_{i}, F_{j}$, $F_{k}$ and $F_{\ell}$ are distinct, to define this linking number. If $\{i, j\}=\{k, \ell\}$, this linking number is the self-linking of the intersection curve that is framed by the surfaces, up to sign. Now, note that $\operatorname{lk}\left(F_{i} \cap F_{j}, F_{i}^{+} \cap F_{\ell}^{+}\right)=\operatorname{lk}\left(F_{i} \cap F_{j}, F_{i}^{+} \cap F_{\ell}\right)$ and that

$$
\operatorname{lk}\left(F_{i}^{+} \cap F_{j}, F_{i} \cap F_{\ell}\right)-\operatorname{lk}\left(F_{i} \cap F_{j}, F_{i}^{+} \cap F_{\ell}\right)= \pm\left\langle F_{i}, F_{j}, F_{\ell}\right\rangle .
$$

Thus if the cardinality of $\{i, j\} \cap\{k, \ell\}$ is 1 , the linking number $\ell\left(\left(F_{i}\right)_{i=1, \ldots 2 n} ; \Gamma ; e\right)$ is well-defined, too. Define $\ell\left(\left(F_{i}\right)_{i=1, \ldots 2 n} ; \Gamma\right)$ as the product over all the unlabelled edges of $\Gamma$ of the $\ell\left(\left(F_{i}\right)_{i=1, \ldots 2 n} ; \Gamma ; e\right)$. Note that $\ell\left(\left(F_{i}\right)_{i=1, \ldots 2 n} ; \Gamma\right)[\Gamma]$ is independent of the orientation of $\Gamma$. Let $\sharp A_{2} t_{3}(\Gamma)$ be the number of automorphisms of $\Gamma$ that preserve its $2 / 3$-labelling.

Theorem 4.4 Let $n$ and $r$ be elements of $\mathbb{N}$. Let $L=\left(K_{i} ; p_{i} / q_{i}\right)_{i \in\{1,2,3, \ldots, r\}}$ be a framed $r$-component algebraically split link in a rational homology sphere $M$ such that for any $\{i, j, k\} \subset\{1,2,3, \ldots, r\}, \mu\left(K_{i}, K_{j}, K_{k}\right)=0$.

Let $\left(F_{i}\right)_{i \in\{1,2,3, \ldots, r\}}$ be a collection of transverse Seifert surfaces for the $K_{i}$ where $F_{i}$ does not meet the $K_{j}$ for $i \neq j$. 
Then with the notation above

$$
\begin{aligned}
& Z_{n}\left(\left[M ;\left(K_{i} ; p_{i} / q_{i}\right)_{i \in\{1, \ldots, r\}}\right]\right) \\
& \quad= \begin{cases}0 & \text { if } r>2 n, \\
\left(\prod_{i=1}^{2 n} \frac{q_{i}}{p_{i}}\right) \sum_{\Gamma \in D_{2 / 3, n}} \frac{\ell\left(\left(F_{i}\right)_{i=1, \ldots 2 n} ; \Gamma\right)}{\sharp A_{2} / 3}[\Gamma] & \text { if } r=2 n\end{cases}
\end{aligned}
$$

where the sum runs over all (isomorphism classes of) 2/3-labelled unoriented Jacobi diagrams $\Gamma$.

When $M$ is a $\mathbb{Z}$-sphere, when the $p_{i}$ are equal to 1 , and when $r$ is greater or equal, than $n$ for Theorem 4.1, than $2 n$ for Theorem 4.4, and than $3 n$ for Theorem 4.3, the left-hand sides of the equalities of these theorems are in $\mathcal{F}_{2 n}^{\mathrm{GH}}$. Since the degree $n$ part of the LMO invariant coincides with $Z_{n}$ on $\mathcal{F}_{2 n}^{\mathrm{GH}}$, these three theorems hold for the LMO invariant as well, in these cases.

Theorem 4.3 and Theorem 4.4 will be proved in Section 11. Their proofs will rely on some clasper calculus performed in Section 10, that will also lead to the following proposition.

Proposition 4.5 Let $L=\left(K_{i}\right)_{i \in\{1,2,3, \ldots, r\}}$ be an $r$-component algebraically split link in a rational homology sphere $M$. Then there exist transverse Seifert surfaces $\Sigma_{i}$ in $M \backslash\left(\bigcup_{j \neq i} K_{j}\right)$ for each component $K_{i}$ of $L$, such that, for any triple $\left(K_{i}, K_{j}, K_{k}\right)$ of components of $L$, the geometric triple intersection of the surfaces $\Sigma_{i}, \Sigma_{j}$ and $\Sigma_{k}$ is made of $\left|\mu\left(K_{i}, K_{j}, K_{k}\right)\right|$ points.

Section 11 also contains an equivalent definition of the Matveev Borromean surgery (or surgery on a $Y$-graph); see Proposition 10.1.

\section{On the polynomial form of the knot surgery formula}

Recall that for any rational homology sphere $M, Z_{0}(M)=1$. Theorem 4.1 implies that for any knot $K$ that bounds a surface $F$ in a rational homology sphere $M$ and for any two coprime integers $p$ and $q$ such that $p \neq 0$,

$$
Z_{1}\left(M\left(K ; \frac{p}{q}\right)\right)-Z_{1}(M)=\frac{q}{2 p}\langle\langle I(F)\rangle\rangle+Z_{1}(L(p,-q)) .
$$

We shall see in Section 12 that Theorem 4.1 also easily implies the following theorem. The first part of this theorem is essentially [13, Proposition 4.1] by Garoufalidis. 
Theorem 5.1 Let $p$ and $q$ be coprime integers such that $p \neq 0$. Let $n \in \mathbb{N}, n \geq 1$. Let $K$ be a knot that bounds a Seifert surface $F$ in a rational homology sphere $M$. Let $F^{i}$ be parallel copies of $F$ for $i \in\{1, \ldots, n\}$, and let $L_{i}$ denote the framed link made of the boundary components of $\bigcup_{j=1}^{i} F_{j}$, where each component is framed by 1 .

Then

$$
Z_{n}\left(M\left(K ; \frac{p}{q+r p}\right)\right)-Z_{n}(M)=\sum_{i=0}^{n} Y_{n, q / p}^{(i)}(K \subset M)\left(r+\frac{q}{p}\right)^{i}
$$

for any $r \in \mathbb{Z}$ where the coefficients $Y_{n, q / p}^{(i)}(K)$ satisfy the following properties:

- $\left.\left.Y_{n, q / p}^{(n)}(K)=\frac{(-1)^{n}}{n !} Z_{n}\left(\left[M ; L_{n}\right]\right)=\frac{1}{n ! 2^{n}} \| \bigsqcup_{i \in\{1, \ldots, n\}} I\left(F^{i}\right)\right)\right\rangle$.

- If $n \geq 2$,

$Y_{n, q / p}^{(n-1)}(K)=\frac{(-1)^{n-1}}{(n-1) !}\left(\left(\frac{n-1}{2}+\frac{q}{p}\right) Z_{n}\left(\left[M ; L_{n}\right]\right)+Z_{n}\left(\left[M\left(K ; \frac{p}{q}\right) ; L_{n-1}\right]\right)\right)$.

- If $n \geq 2, p^{c}\left(Y_{n, q / p}^{(n-1)}\right)=Y_{n, q / p}^{(n-1) c}$ does not depend on $p$ and $q$.

- If $i \leq n-1, Y_{n, q / p}^{(i)}$ only depends on $q / p \bmod \mathbb{Z}$.

- If $U$ bounds a disk in $M$, then $Y_{n, q / p}^{(i)}(U \subset M)=0$ if $i>0$ and

$$
Y_{n, q / p}^{(0)}(U \subset M)=Z_{n}(M \sharp L(p,-q))-Z_{n}(M) .
$$

- $Y_{n, 0}^{(0)}(K \subset M)=0$.

- $Y_{n, q / p}^{(i)}(K \subset M)=(-1)^{i+n} Y_{n,-q / p}^{(i)}(K \subset-M)$.

A singular knot is an immersion of $S^{1}$ in a 3 -manifold whose only multiple points are transverse double points like $\chi$.

Such a double point can be removed in a positive way $\lambda$ or in a negative way $\searrow$.

Let $K^{s}$ be a singular knot with $k$ double points in a rational homology sphere. Fix a bijection from $\{1, \ldots, k\}$ to its set of double points. For $I \subset\{1, \ldots, k\}$, let $K_{I}$ be the desingularisation of $K^{s}$ such that the singular points in the image of $I$ have been removed in a negative way, and the singular points outside the image of $I$ have become positive. If $y$ is a knot invariant with values in an abelian group, set

$$
y\left(K^{S}\right)=\sum_{I \subset\{1, \ldots, k\}}(-1)^{\sharp I} y\left(K_{I}\right) .
$$


Remark 5.2 It may happen that we do not know whether $Z_{n}\left(M\left(K_{I} ; \frac{p}{q+p r}\right)\right)$ is polynomial in $r$ for a given $I$, but that we know that

$$
\sum_{I \subset\{1, \ldots, k\}}(-1)^{\sharp I} Z_{n}\left(M\left(K_{I} ; \frac{p}{q+p r}\right)\right)
$$

Proposition 5.3 For any singular knot $K^{s}$ in a rational homology sphere with $k$ double points, for any integers $n, i, q$ and $p$, with $0 \leq i \leq n$,

- $Y_{n, q / p}^{(i)}\left(K^{s}\right)=0$ if $k>2 n$,

- $Y_{n, q / p}^{(i)}\left(K^{s}\right)=0$ if $k>2 n-1$ and if $i<n$.

In other words, $Y_{n, q / p}^{(i)}$ is a knot invariant of degree at most $2 n$ with respect to the crossing changes, and if $i<n, Y_{n, q / p}^{(i)}$ is a knot invariant of degree at most $(2 n-1)$ with respect to the crossing changes.

Two disjoint pairs of points in $S^{1}$ are said to be unlinked if they bound disjoint intervals in $S^{1}$. Otherwise, they are said to be linked. Two double points of a singular knot are said to be linked if their preimages are linked.

Associate a symmetric linking matrix $\left[\ell_{i j}\left(K^{s}\right)\right]_{i, j \in\{1,2, \ldots, k\}}$ with a singular knot $K^{s}$ with $k$ pairwise unlinked double points numbered from 1 to $k$ in the following way. Each double point $i \backslash$ can be smoothed to transform the knot into two oriented singular knots $K_{i}^{s \prime}$ and $K_{i}^{s \prime \prime}$ :

Set

$$
\begin{aligned}
& \smile K_{i}^{s \prime} \\
& ح K_{i}^{s \prime \prime}
\end{aligned}
$$

Set

$$
\ell_{i i}\left(K^{s}\right)=\operatorname{lk}\left(K_{i}^{s \prime}, K_{i}^{s \prime \prime}\right) .
$$

If $i$ and $j$ label two unlinked double points, let $K_{i}^{s, j}$ be the curve among $K_{i}^{s \prime}$ and $K_{i}^{s \prime \prime}$ that does not contain the double point labeled by $j$, then $\ell_{i j}\left(K^{s}\right)=\operatorname{lk}\left(K_{i}^{s, j}, K_{j}^{s, i}\right)$ if $i \neq j$.

Proposition 5.4 For any singular knot $K^{s}$ in a rational homology sphere with $k$ pairwise unlinked double points, for any integers $n, i, q$ and $p$, with $0 \leq i \leq n$, and $n \geq 1$,

- if $k>n, Y_{n, q / p}^{(i)}\left(K^{s}\right)=0$,

- if $k=n, Y_{n, q / p}^{(i)}\left(K^{s}\right)=Y_{n, 0}^{(i)}\left(K^{s}\right)$ is an explicit homogeneous polynomial of degree $i$ in the coefficients of the linking matrix of $K^{s}$, and $Y_{n, q / p}^{(0)}\left(K^{s}\right)=0$. 
Proposition 12.2 will give explicit examples of computations of the above homogeneous polynomials.

\section{A few formulae for the Casson-Walker invariant}

Set $\lambda=W_{1} \circ Z_{1}$ where $W_{1}(\Theta)=2$. According to [26], $\lambda$ is the Casson-Walker invariant as normalized by Casson for $\mathbb{Z}$-spheres (see Akbulut and McCarthy [1] and Guillou and Marin [18;29]), $\lambda$ is half the Walker invariant as normalized in [35], and $\lambda$ coincides with $\bar{\lambda} /\left|H_{1}(M)\right|$, where $\left|H_{1}(M)\right|$ denotes the cardinality of $H_{1}(M ; \mathbb{Z})$ for $\mathbb{Q}$-spheres and $\bar{\lambda}$ is the extension of $\left|H_{1}(M)\right| \lambda$ to oriented closed 3-manifolds that is denoted by $\lambda$ in [27].

A rationally algebraically split link is a link whose components do not link each other algebraically (ie have linking number zero). The following proposition gives formulae that generalize Theorem 4.1, Theorem 4.3 and Theorem 4.4 in the degree 1 case $(n=1)$ for rationally algebraically split links.

The order of a knot $K$ in a rational homology sphere is the smallest positive integer $O_{K}$ such that $O_{K} K$ is null-homologous. A primitive curve on a torus $S^{1} \times S^{1}$ is a nonseparating simple closed curve on the torus. A primitive satellite of a knot is a primitive curve on the boundary $\partial N(K)$ of its tubular neighborhood. A surgery on a knot $K$ is determined by a primitive satellite $\mu$ (oriented arbitrarily) of the knot that will bound a disk inside the surgered torus after surgery. If $m_{K}$ is the meridian of $K$, the isotopy class of such a curve is determined by the pair

$$
\left(p_{K}=1 \mathrm{k}(\mu, K), q_{K}=\left\langle m_{K}, \mu\right\rangle_{\partial N(K)}\right)
$$

and the surgery coefficient is $p_{K} / q_{K}$.

For any order $d$ component $K$ of a rationally algebraically split link $L$, there exists an embedded surface $\Sigma$ in the complement of $L$ whose boundary $\partial \Sigma$ is made of essential parallel curves of the boundary $\partial N(K)$ of the tubular neighborhood $N(K)$ of $K$ such that $\partial \Sigma$ is homologous to $d$ parallels of $K$ in $N(K)$. Let $H_{1}(\Sigma) / H_{1}(\partial \Sigma)$ denote the quotient of $H_{1}(\Sigma)$ by the image of $H_{1}(\partial \Sigma)$ under the map induced by the inclusion. Let $B_{s}=\left(x_{i}, y_{i}\right)_{i \in\{1, \ldots, g\}}$ be a symplectic basis of $H_{1}(\Sigma) / H_{1}(\partial \Sigma)$, define

$$
I(\Sigma)=\sum_{(j, k) \in\{1,2, \ldots, g\}^{2}} x_{j} y_{j} y_{k}^{+} x_{k}^{+} .
$$

If $W_{n}: \mathcal{A}_{n} \rightarrow \mathbb{Q}$ is a linear form, then $W_{n}(\langle\langle\cdot\rangle\rangle)$ will also be denoted by $\langle\langle\cdot\rangle\rangle_{W_{n}}$. 
For example,

$$
\langle\langle I(\Sigma)\rangle\rangle_{W_{1}}=2 \sum_{(j, k) \in\{1,2, \ldots, g\}^{2}}\left(\operatorname{lk}\left(x_{j}, x_{k}^{+}\right) \operatorname{lk}\left(y_{j}, y_{k}^{+}\right)-\operatorname{lk}\left(x_{j}, y_{k}^{+}\right) \operatorname{lk}\left(y_{j}, x_{k}^{+}\right)\right) .
$$

Proposition 6.1 Let $n$ be an integer. Set $N=\{1, \ldots, n\}$. Let $L=\left(K_{i} ; p_{i} / q_{i}\right)_{i \in N}$ be a framed rationally algebraically split link in a rational homology sphere $M$. Let $d_{i}$ be the order of $K_{i}$ in $H_{1}(M)$, let $\Sigma_{i}$ be a surface of $M \backslash L$ whose boundary is made of essential parallel curves of $\partial N\left(K_{i}\right)$ and is homologous to $d_{i} K_{i}$ in $N\left(K_{i}\right)$. If $n=1$, assume that the $\mathbb{Q} / \mathbb{Z}$-self-linking number of $K_{1}$ is zero.

Then

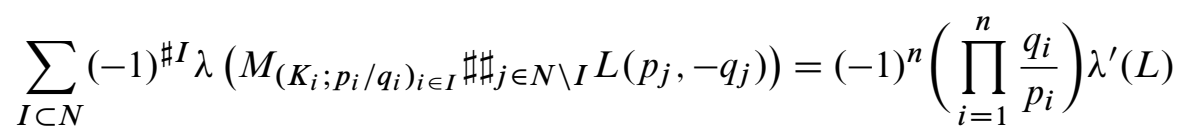

where

$$
\lambda^{\prime}(L)= \begin{cases}\frac{\left.\left\langle I\left(\Sigma_{1}\right)\right\rangle\right\rangle_{W_{1}}}{2 d_{1}^{2}}+\frac{1}{12}-\frac{1}{12 d_{1}^{2}} & \text { if } n=1, \\ \frac{\left.\left\langle I\left(\Sigma_{1}\right) \subset M\left(K_{2} ; 1\right)\right\rangle\right\rangle_{W_{1}}}{2 d_{1}^{2}}-\frac{\left\langle I\left(\Sigma_{1}\right) \subset M\right\rangle_{W_{1}}}{2 d_{1}^{2}} & \text { if } n=2, \\ =-\frac{1 \mathrm{k}\left(\Sigma_{1} \cap \Sigma_{2},\left(\Sigma_{1} \cap \Sigma_{2}\right) \|\right)}{d_{1}^{2} d_{2}^{2}} & \\ \frac{\left\langle\Sigma_{1}, \Sigma_{2}, \Sigma_{3}\right\rangle^{2}}{d_{1}^{2} d_{2}^{2} d_{3}^{2}} & \text { if } n=3, \\ 0 & \text { if } n \geq 4,\end{cases}
$$

and, if $n>1$,

$$
\sum_{I \subset N}(-1)^{\sharp I} \lambda\left(M_{\left(K_{i} ; p_{i} / q_{i}\right)_{i \in I} \sharp \sharp H_{j \in N} \backslash I} L\left(p_{j},-q_{j}\right)\right)=\sum_{I \subset N}(-1)^{\sharp I} \lambda\left(M_{\left(K_{i} ; p_{i} / q_{i}\right)_{i \in I}}\right) .
$$

This proposition is proved in Section 13. Under its hypotheses, we then obviously have the following equalities

$$
\lambda^{\prime}\left(K_{1} \subset M\left(K_{2}, p / q\right)\right)-\lambda^{\prime}\left(K_{1} \subset M\right)=\frac{q}{p} \lambda^{\prime}\left(K_{1}, K_{2}\right)
$$

and

$$
\begin{aligned}
& \lambda^{\prime}\left(K_{1} \subset M\left(\left(K_{2} ; p_{2} / q_{2}\right),\left(K_{3} ; p_{3} / q_{3}\right)\right)\right) \\
& -\lambda^{\prime}\left(K_{1} \subset M\left(K_{2} ; p_{2} / q_{2}\right)\right)-\lambda^{\prime}\left(K_{1} \subset M\left(K_{3} ; p_{3} / q_{3}\right)\right)+\lambda^{\prime}\left(K_{1} \subset M\right) \\
& =\frac{q_{2} q_{3}}{p_{2} p_{3}} \lambda^{\prime}\left(K_{1}, K_{2}, K_{3}\right) .
\end{aligned}
$$

Then the variation of linking numbers under surgery recalled in Lemma 9.5 easily implies the following proposition (see also the proof of Lemma 13.4). 
Proposition 6.2 Let $\left(K_{1}, K_{2}, K_{3}\right)$ be a rationally algebraically split link in a rational homology sphere $M$. Let $d_{i}$ be the order of $K_{i}$ in $H_{1}(M)$, let $\Sigma_{i}$ be a surface of $M \backslash L$ whose boundary is made of essential parallel curves of $\partial N\left(K_{i}\right)$ and is homologous to $d_{i} K_{i}$ in $N\left(K_{i}\right)$. Then

$$
\begin{gathered}
\lambda^{\prime}\left(K_{1}, K_{2}\right)=-\frac{1}{4}\left\langle\left\langle\frac{1}{d_{1}^{2}} I\left(\Sigma_{1}\right) K_{2}-K_{2} \|\right\rangle_{W_{1}}=-\frac{1}{4}\left\langle\left\langle\frac{1}{d_{2}^{2}} I\left(\Sigma_{2}\right) K_{1}-K_{1 \|} \|_{W_{1}},\right.\right.\right. \\
\lambda^{\prime}\left(K_{1}, K_{2}, K_{3}\right)=\frac{1}{8 d_{1}^{2}}\left\langle\left\langle I\left(\Sigma_{1}\right) K_{2}-K_{2 \|} K_{3}-K_{3 \|}\right\rangle\right\rangle_{W_{1}} .
\end{gathered}
$$

Proposition 6.3 If $K^{s}$ is a singular knot with one double point, then

$$
\lambda^{\prime}\left(K^{s}\right)=\ell_{11}\left(K^{s}\right) .
$$

The easy proof of this well-known proposition is also given in Section 13.

\section{On the knot surgery formula for the degree 2 invariant $\lambda_{2}$}

Consider the degree 2 invariant

where

$$
\begin{aligned}
& \lambda_{2}=W_{2} \circ Z_{2}^{c} \\
& W_{2}(\$)=1 \\
& W_{2}(\varnothing)=2 .
\end{aligned}
$$

The invariant $\lambda_{2}$ is invariant under orientation change and additive under connected sum.

Theorem 7.1 There exists a function $c$ from $\mathbb{Q} / \mathbb{Z}$ to $\mathbb{Q}$ such that $c(0)=0, c(q / p)=$ $c(-q / p)$ and the following assertions hold. Let $r=q / p \in \mathbb{Q} \backslash\{0\}$, where $p$ and $q$ are coprime integers. Let $K$ be a knot that bounds a Seifert surface $F$ in a rational homology sphere $M$. Let $F^{1}$ and $F^{2}$ be two parallel copies of $F$. Then

$$
\lambda_{2}(M(K ; 1 / r))-\lambda_{2}(M)=\lambda_{2}^{\prime \prime}(K) r^{2}+w_{3}(K) r+C(K ; q / p)+\lambda_{2}(L(p,-q))
$$

where

$$
\begin{aligned}
\lambda_{2}^{\prime \prime}(K) & \left.\left.=\frac{1}{8} \| \bigsqcup_{i \in\{1,2\}}\left(I\left(F^{i}\right)\right)\right\rangle\right\rangle_{W_{2}} \\
w_{3}(K \subset M) & =-w_{3}(K \subset(-M))
\end{aligned}
$$

and $C(\cdot ; q / p)$ is an invariant of null-homologous knots that only depends on $q / p$ $\bmod \mathbb{Z}$, such that 
- $C(K ; 0)=0$,

- if $K$ bounds a surface whose $H_{1}$ vanishes in $H_{1}(M)$, then $C(K ; q / p)=$ $c(q / p) \lambda^{\prime}(K)$.

Furthermore, if $K^{s}$ is a singular knot with two unlinked double points, then

$$
w_{3}\left(K^{s}\right)=-\frac{\ell_{12}\left(K^{s}\right)}{2} \text { and } C\left(K^{s} ; q / p\right)=0 .
$$

Like all the statements in this section, the above theorem will be proved in Section 14 .

Proposition 7.2 Let $K^{s}$ be a singular knot with one double point in a rational homology sphere. Let $K^{+}$and $K^{-}$be its two desingularisations, and let $K^{\prime}$ and $K^{\prime \prime}$ be the two knots obtained from $K^{s}$ by smoothing the double point. Assume that $K^{\prime}$ and $K^{\prime \prime}$ are null-homologous, then

$$
w_{3}\left(K^{+}\right)-w_{3}\left(K^{-}\right)=\frac{\lambda^{\prime}\left(K^{\prime}\right)+\lambda^{\prime}\left(K^{\prime \prime}\right)}{2}-\frac{\lambda^{\prime}\left(K^{+}\right)+\lambda^{\prime}\left(K^{-}\right)+1 \mathrm{k}^{2}\left(K^{\prime}, K^{\prime \prime}\right)}{4} .
$$

Let $x$ denote a two-strand braid with $|x|$ vertical juxtapositions of the pattern $\mathrm{Y}$ if $x>0$ and $|x|$ vertical juxtapositions of the pattern $\backslash$ if $x<0$.

Let $x, y$ and $z$ be three odd numbers. Let $K(x, y, z)$ be the pretzel knot of Figure 2 that bounds a genus one Seifert surface $\Sigma$ whose thickening $H$ coincides with the thickening of the twice punctured disk next to $K(x, y, z) . H$ is a genus two handlebody whose boundary is equipped with curves $X, Y$ and $Z$ that bound disks in its exterior.
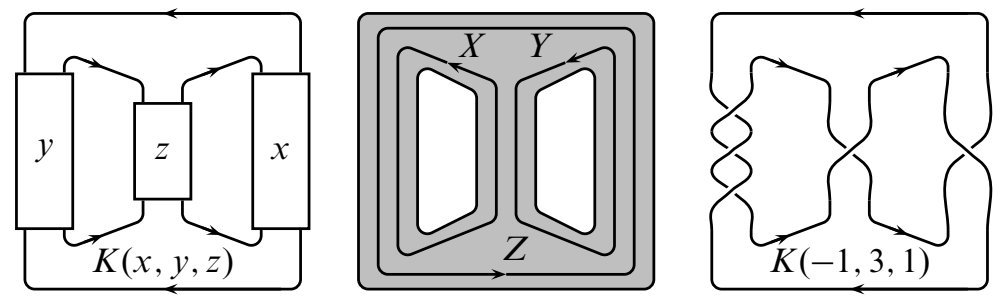

Figure 2

Note that any genus one knot that bounds a genus one surface, whose $H_{1}$ goes to 0 in $H_{1}(M)$, may be written as the image of $K(x, y, z)$ under an embedding $\phi$ of $H$ into $M$ that maps $X$ and $Y$ to 0 in $H_{1}(M \backslash \phi(H))$. 
Proposition 7.3 Let $\phi$ be an embedding of $H$ in a rational homology sphere such that $\phi(X)$ and $\phi(Y)$ are null-homologous in the exterior of $\phi(H)$. Then

$$
\begin{aligned}
w_{3}(\phi(K(x, y, z)))= & w_{3}(K(x, y, z)) \\
& -\frac{x}{2} \lambda^{\prime}(\phi(X))-\frac{y}{2} \lambda^{\prime}(\phi(Y))-\frac{z}{2} \lambda^{\prime}(\phi(Z))+\frac{3}{2} \lambda^{\prime}(\phi(X), \phi(Y))
\end{aligned}
$$

and $w_{3}(K(x, y, z))=\frac{x^{2}(y+z)+y^{2}(x+z)+z^{2}(x+y)}{32}+\frac{x y z}{8}+\frac{x+y+z}{16}$

where the quantities $\lambda^{\prime}(\phi(X))$ and $\lambda^{\prime}(\phi(X), \phi(Y))$ are defined in several equivalent ways in Section 6.

\section{Proof of the Lagrangian-preserving surgery formula}

In this section, we prove Theorem 3.1 by proving that its formula is equivalent to the formula of [26] (or [3] for the case of integral homology spheres). We first rewrite the right-hand side of the formula of Theorem 3.1.

Let $g(i)$ be the genus of $A_{i}$. Let $\left(a_{1}^{i}, a_{2}^{i}, \ldots, a_{g(i)}^{i}\right)$ be a basis of $\mathcal{L}_{A_{i}}$, and let $z_{1}^{i}, \ldots, z_{g(i)}^{i}$ be homology classes of $\partial A_{i}$, such that $\left\langle a_{j}^{i}, z_{k}^{i}\right\rangle_{\partial A}=\delta_{j k}$. Let $F$ be the set of maps $f$ from $\{1, \ldots, 2 n\} \times\{1,2,3\}$ to $\mathbb{N}$ such that $1 \leq f(i, 1)<f(i, 2)<$ $f(i, 3) \leq g(i)$. Let $P$ be the set of pairings $p$ of the disjoint union $G^{0}$ of $2 n$ tripods as in Figure 3, that pair a univalent vertex of some tripod to a univalent vertex of a different tripod.

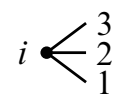

Figure 3: The tripod associated with $i \in\{1,2, \ldots, 2 n\}$

Let $p \in P$. The half-edges of $G_{p}^{0}$ are naturally labeled in $\{1, \ldots, 2 n\} \times\{1,2,3\}$. Assume that some $(f \in F)$ is given. With a half-edge of $G_{p}^{0}$ labeled by $(i, j)$ that belongs to the tripod $i$, associate the curve $z_{f(i, j)}^{i}$ of $\partial A_{i}$. Then with an edge of $G_{p}^{0}$, associate the linking number of the curves associated with its two half-edges, and define 
$\operatorname{lk}(p ; f)$ as the product over the edges of these linking numbers. Set

and

$$
c(p ; f)=\operatorname{lk}(p ; f) \prod_{i=1}^{2 n} \mathcal{I}_{A_{i} A_{i}^{\prime}}\left(a_{f(i, 1)}^{i}, a_{f(i, 2)}^{i}, a_{f(i, 3)}^{i}\right),
$$

Then

$$
c(p)=\sum_{f \in F} c(p ; f) \text {. }
$$

$$
\left\|\bigsqcup_{i \in\{1, \ldots, 2 n\}} T\left(\mathcal{I}_{A_{i} A_{i}^{\prime}}\right)\right\|_{n}=\sum_{p \in P} c(p)\left[G_{p}^{0}\right] .
$$

Let $D$ be the set of (isomorphism classes of) unoriented Jacobi diagrams of degree $n$. Consider a Jacobi diagram $\Gamma$ of $D$. Let $P(\Gamma)$ be the set of the pairings $p$ of $P$ such that $G_{p}^{0}$ is isomorphic to $\Gamma$ as an unoriented Jacobi diagram. Then

$$
\left\|\bigsqcup_{i \in\{1, \ldots, 2 n\}} T\left(\mathcal{I}_{A_{i} A_{i}^{\prime}}\right)\right\|_{n}=\sum_{\Gamma \in D} \sum_{p \in P(\Gamma)} c(p)\left[G_{p}^{0}\right] .
$$

Fix $\Gamma$ in $D$. Let $B(\Gamma)$ be the set of bijections $b$ from the set $H(\Gamma)$ of half-edges of $\Gamma$ to $\{1, \ldots, 2 n\} \times\{1,2,3\}$ that map any half-edge $c$ of a vertex $v(c)$ to three images with the same first coordinate $b_{1}(c)=b_{1}(v(c))$. An element $b$ of $B(\Gamma)$ determines a pairing $p(b)$ of $P(\Gamma)$, and the number of elements of $B(\Gamma)$ that determine the same pairing is the number of automorphisms of $\Gamma$.

$$
\sum_{p \in P(\Gamma)} c(p)\left[G_{p}^{0}\right]=\sum_{b \in B(\Gamma)} \frac{c(p(b))}{\sharp \operatorname{Aut}(\Gamma)}\left[G_{p(b)}^{0}\right]=\sum_{b \in B(\Gamma), f \in F} \frac{c(p(b) ; f)}{\sharp \operatorname{Aut}(\Gamma)}\left[G_{p(b)}^{0}\right] .
$$

Let $G(\Gamma)$ be the set of injections $g$ from the set $H(\Gamma)$ of half-edges of $\Gamma$ to

$$
\{(i, j) \in\{1, \ldots, 2 n\} \times \mathbb{N} ; 1 \leq j \leq g(i)\}
$$

that map the three half-edges of a vertex to three images with the same first coordinate, and that induce a bijection from $V(\Gamma)$ to $\{1, \ldots, 2 n\}$. An injection $g$ of $G(\Gamma)$ yields a natural bijection $b(g)$ of $B(\Gamma)$ and a map $f(g)$ of $F$ such that $g(c)=\left(b_{1}(c), f(g) \circ\right.$ $b(g)(c))$. Furthermore, such a $g$ orders the three half-edges of a vertex, and hence yields an orientation $o(g)$ of $\Gamma$.

$$
\sum_{p \in P(\Gamma)} c(p)\left[G_{p}^{0}\right]=\sum_{g \in G(\Gamma)} \frac{c(p(b(g)) ; f(g))}{\sharp \operatorname{Aut}(\Gamma)}[(\Gamma, o(g))]
$$

Let $g \in G(\Gamma)$, its first coordinate $b_{1}(g)$ induces a bijection from $V(\Gamma)$ to $\{1, \ldots, 2 n\}$. Number the three half-edges of any vertex $w$ of $\Gamma$ with a bijection $b(w): v^{-1}(w) \rightarrow$ $\{1,2,3\}$, arbitrarily. This orients $\Gamma$ and equips each injection $g \in G(\Gamma)$ with a sign 
that is +1 if $o(g)$ coincides with this orientation of $\Gamma$ (except for an even number of vertices) and $(-1)$ otherwise. Furthermore, $g$ yields summands of

$$
\mathcal{I}\left(A_{i}, A_{i}^{\prime}\right)=\sum_{g_{i}:\{1,2,3\} \rightarrow\{1,2, \ldots, g(i)\}} \mathcal{I}_{A_{i} A_{i}^{\prime}}\left(a_{g_{i}(1)}^{i}, a_{g_{i}(2)}^{i}, a_{g_{i}(3)}^{i}\right) z_{g_{i}(1)}^{i} \otimes z_{g_{i}(2)}^{i} \otimes z_{g_{i}(3)}^{i}
$$

where $g\left(b\left(b_{1}(g)^{-1}(i)\right)^{-1}(j)\right)=\left(i, g_{i}(j)\right)$. Note that the sign of an injection $g$ is +1 if the number of vertices $b_{1}(g)^{-1}(i)$ where the cyclic order induced by $g_{i}$ does not coincide with the cyclic order induced by $b\left(b_{1}(g)^{-1}(i)\right)$ is even, and $(-1)$, otherwise. This shows that for any bijection $\sigma$ from $V(\Gamma)$ to $\{1, \ldots, 2 n\}$,

$$
\sum_{g \in G(\Gamma) ; b_{1}(g)=\sigma} c(p(b(g)) ; f(g))[(\Gamma, o(g))]=\operatorname{lk}\left(\left(A_{i}, A_{i}^{\prime}\right)_{i=1, \ldots, 2 n} ; \Gamma ; \sigma\right)[\Gamma]
$$

with the notation of [3] or [26].

\section{A direct proof of the formula for boundary links}

\subsection{A Lagrangian-preserving surgery associated with a Seifert surface}

Let $\Sigma$ be an oriented Seifert surface of a knot $K$ in a manifold $M$. Consider an annular neighborhood $[-3,0] \times K$ of $(\{0\} \times K)=K=\partial \Sigma$ in $\Sigma$, a small disk $D$ inside $]-2,-1[\times K$, and an open disk $d$ in the interior of $D$. Let $F=\Sigma \backslash d$. Let $h_{F}$ be the composition of the two left-handed Dehn twists on $F$ along $c=\partial D$ and $K_{2}=\{-2\} \times K$ with the right-handed one along $K_{1}=\{-1\} \times K$. See Figure 4 .

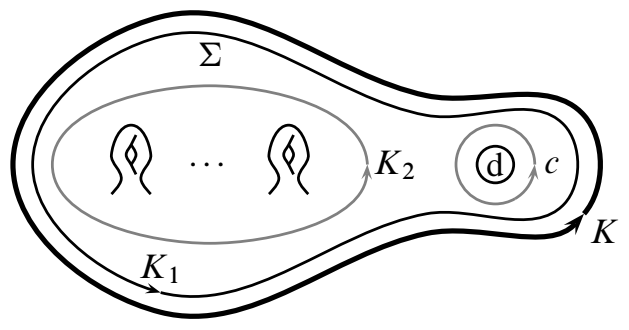

Figure 4

See $F$ as $F \times\{0\}$ in the boundary of the handlebody $A_{F}=F \times[-1,0]$ of $M$. Extend $h_{F}$ to a homeomorphism $h_{A}$ of $\partial A_{F}$ by defining it as the identity outside $F \times\{0\}$. Let $A_{F}^{\prime}$ be a copy of $A_{F}$. Identify $\partial A_{F}^{\prime}$ with $\partial A_{F}$ with

$$
h_{A}: \partial A_{F}^{\prime} \rightarrow \partial A_{F} \text {. }
$$


Define the surgery associated with $\Sigma$ as the surgery associated with $\left(A_{F}, A_{F}^{\prime}\right)$ (or $\left.\left(A_{F}, A_{F}^{\prime} ; h_{A}\right)\right)$. If $\iota$ denotes the embedding from $\partial A_{F}$ to $M$. This surgery replaces

by

$$
\begin{aligned}
M & =\left(M \backslash \operatorname{Int}\left(A_{F}\right)\right) \cup_{\iota} A_{F} \\
M_{F} & =\left(M \backslash \operatorname{Int}\left(A_{F}\right)\right) \cup_{\iota h_{A}} A_{F}^{\prime} .
\end{aligned}
$$

Proposition 9.1 With the notation above, the surgery $\left(A_{F}, A_{F}^{\prime}\right)$ associated with $\Sigma$ is a Lagrangian-preserving surgery with the following properties. There is a homeomorphism from $M_{F}$ to $M$

- that extends the identity of

$$
M \backslash([-3,0] \times K \times[-1,0]),
$$

- that transforms a curve passing through $d \times[-1,0]$ by a band sum with $K$,

- that transforms a 0 -framed meridian $m$ of $K$ passing through $d \times[-1,0]$ into a 0 -framed copy of $K$ isotopic to the framed curve $h_{A}^{-1}(m)$ of Figure 5.
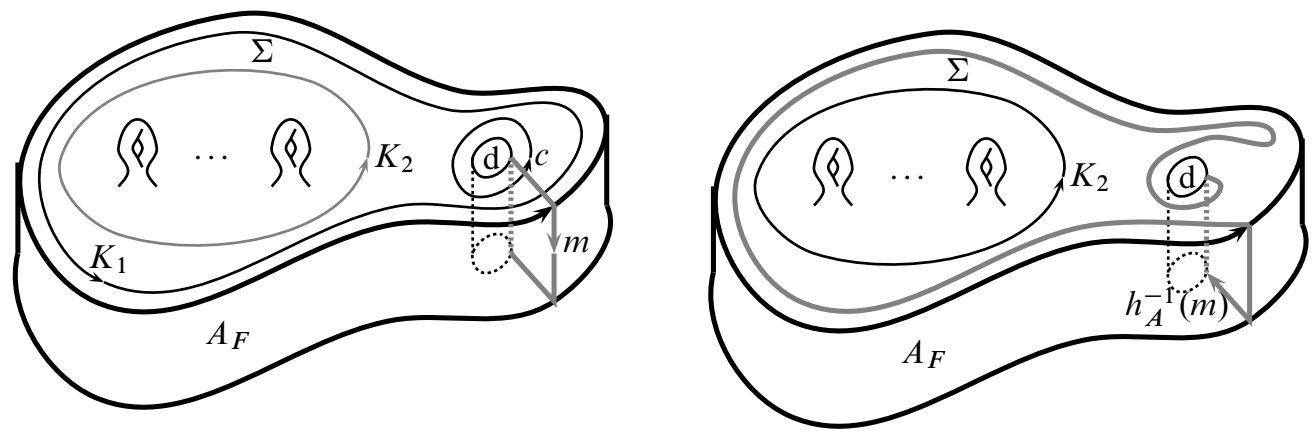

Figure 5

Proof Observe that $h_{F}$ extends to $\Sigma \times[-1,0]$ as

$$
\begin{aligned}
h: \Sigma \times[-1,0] & \rightarrow \Sigma \times[-1,0] \\
(\sigma, t) & \mapsto h(\sigma, t)=\left(h_{t}(\sigma), t\right)
\end{aligned}
$$

where $h_{0}$ is the extension of $h_{F}$ by the identity on $d$ that is isotopic to the identity,

- $h_{-1}$ is the identity of $\Sigma$,

- $h_{t}$ coincides with the identity outside $[-5 / 2,-1 / 2] \times K\left(S^{1}\right)$,

- $h_{t}$ is defined as follows on $[-5 / 2,-1 / 2] \times K\left(S^{1}\right)$. 
- When $t \leq-1 / 2$, then $h_{t}$ describes the following isotopy between $\left(h_{-1}=\right.$ identity) and the composition $h_{-1 / 2}$ of the left-handed Dehn twist along $K_{2}$ with the righthanded Dehn twist along $K_{1}$, where the first twist is supported on $[-5 / 2,-2] \times K\left(S^{1}\right)$ and the latter is supported on $[-1,-1 / 2] \times K\left(S^{1}\right)$ :

$$
h_{t}(u, K(z))= \begin{cases}(u, K(z \exp (i(2 t+2)(4 \pi(u+5 / 2))))) & \text { if }-5 / 2 \leq u \leq-2 \\ (u, K(z \exp (i(2 t+2)(2 \pi)))) & \text { if }-2 \leq u \leq-1 \\ (u, K(z \exp (-i(2 t+2)(4 \pi(u+1 / 2))))) & \text { if }-1 / 2 \geq u \geq-1\end{cases}
$$

- When $t \geq-1 / 2$, then $h_{t}$ coincides with $h_{-1 / 2}$ outside the disk $D$ whose elements will be written as $D(z \in \mathbb{C})$, with $|z| \leq 1$. The elements of $d$ will be the $D(z)$ for $|z|<1 / 2$. On $D, h_{t}$ will describe the isotopy between the identity and the composition $h_{0}$ of the left-handed Dehn twist along $\partial D$ located on $\{D(z) ; 1 / 2 \leq|z| \leq 1\}$ and a negative twist of $d$.

$$
\begin{aligned}
h_{t}(u, K(z)) & = \begin{cases}(u, K(z \exp (i(4 \pi(u+5 / 2))))) & \text { if } u \leq-2, \\
(u, K(z)) & \text { if }-2 \leq u \leq-1,(u, K(z)) \notin D, \\
(u, K(z \exp (-i(4 \pi(u+1 / 2))))) & \text { if } u \geq-1,\end{cases} \\
h_{t}(z \in D) & = \begin{cases}z \exp (i \pi(2 t+1) 4(|z|-1)) & \text { if }|z| \geq 1 / 2, \\
z \exp (-2 i \pi(2 t+1)) & \text { if }|z| \leq 1 / 2 .\end{cases}
\end{aligned}
$$

Now, $M_{F}$ is naturally homeomorphic to

$$
(M \backslash \operatorname{Int}(\Sigma \times[-1,0])) \cup_{h_{\mid \partial(\Sigma \times[-1,0])}}(\Sigma \times[-1,0])
$$

that maps to $M$ by the identity outside $\Sigma \times[-1,0]$ and by $h$ on $\Sigma \times[-1,0]$, homeomorphically. Therefore, we indeed have a homeomorphism from $M_{F}$ to $M$ that is the identity outside $[-3,0] \times K \times[-1,0]$ and that maps $d \times[-1,0]$ to a cylinder that runs along $K$ before being negatively twisted. In particular, looking at the action of the homeomorphism on a framed arc $x \times[-1,0]$ where $x$ is on the boundary of $d$ shows that the meridian $m$ with its framing induced by the boundary of $A_{F}$ is mapped to a curve isotopic to $h_{A}^{-1}(m)$ in a tubular neighborhood of $K$ with the framing induced by the boundary of $A_{F}$.

Now, $H_{1}\left(\partial A_{F}\right)$ is generated by the generators of $H_{1}(\Sigma) \times\{0\}$, the generators of $H_{1}(\Sigma) \times\{-1\}$, and the homology classes of $c=\partial D$ and $m$. Among them, only the class of $m$ could be affected by $h_{A}$, and it is not. Therefore $h_{A}$ acts trivially on $H_{1}\left(\partial A_{F}\right)$, and the defined surgery is an LP-surgery.

Let $F \times[-1,2]$ be an extension of the previous neighborhood of $F$, and let $B_{F}=$ $F \times[1,2]$. Define the homeomorphism $h_{B}$ of $\partial B_{F}$ as the identity anywhere except 
on $F \times\{1\}$ where it coincides with the homeomorphism $h_{F}$ of $F$ with the obvious identification.

Let $B_{F}^{\prime}$ be a copy of $B_{F}$. Identify $\partial B_{F}^{\prime}$ with $\partial B_{F}$ with

$$
h_{B}: \partial B_{F}^{\prime} \rightarrow \partial B_{F} .
$$

Define the inverse surgery associated with $\Sigma$ as the surgery associated with $\left(B_{F}, B_{F}^{\prime}\right)$ (or $\left(B_{F}, B_{F}^{\prime} ; h_{B}\right)$ ). Note that the previous study can be used for this surgery by using the central symmetry of $[-1,2]$.

Then, we have the following obvious lemma that justifies the terminology.

Lemma 9.2 With the notation above, performing the two surgeries $\left(B_{F}, B_{F}^{\prime}\right)$ and $\left(A_{F}, A_{F}^{\prime}\right)$ affects neither $M$ nor the curves in the complement of $F \times[-1,2]$, while performing one of them changes a 0 -framed meridian of $K$ passing through $d \times[-1,2]$ into a 0 -framed copy of $\pm K$.

Lemma 9.3 Let $\left(x_{i}, y_{i}\right)_{i=1, \ldots, g}$ be a symplectic basis of $\Sigma$, then the tripod combination $T\left(\mathcal{I}_{A_{F} A_{F}^{\prime}}\right)$ associated to the surgery $\left(A_{F}, A_{F}^{\prime}\right)$ is:

$$
T\left(\mathcal{I}_{A_{F} A_{F}^{\prime}}\right)=-\sum_{i=1}^{g} \leftarrow \begin{aligned}
& y_{i} \\
& x_{i}
\end{aligned}
$$

For a curve $c$ of $F$, let $c^{+}$denote $c \times\{1\}$. The tripod combination $T\left(\mathcal{I}_{B_{F} B_{F}^{\prime}}\right)$ associated to the surgery $\left(B_{F}, B_{F}^{\prime}\right)$ is:

$$
T\left(\mathcal{I}_{\boldsymbol{B}_{F} \boldsymbol{B}_{F}^{\prime}}\right)=\sum_{i=1}^{g}<_{c^{+}}^{y_{i}^{+}} x_{i}^{+}
$$

Proof For a curve $c$ of $F, c^{-}$denotes $c \times\{-1\}$. In order to compute the intersection form of $\left(A_{F} \cup-A_{F}^{\prime}\right)$, use the basis $\left(m,\left(x_{i}-x_{i}^{-}, y_{i}-y_{i}^{-}\right)_{i=1, \ldots, g}\right)$ of the Lagrangian of $A_{F}$. Its dual basis is $\left(c,\left(y_{i},-x_{i}\right)_{i=1, \ldots, g}\right)$. Note that the only curve of the Lagrangian basis that is modified by $h_{A}$ is $m$, and that $h_{A}(m)=m K_{2}^{-1}$. The isomorphism $\partial_{M V}^{-1}$ from $\mathcal{L}_{A_{F}}$ to $H_{2}\left(A_{F} \cup-A_{F}^{\prime}\right)$ satisfies:

$$
\begin{aligned}
& \partial_{M V}^{-1}\left(x_{i}-x_{i}^{-}\right)=S\left(x_{i}\right)=-\left(x_{i} \times[-1,0]\right) \cup\left(x_{i} \times[-1,0] \subset A_{F}^{\prime}\right) \\
& \partial_{M V}^{-1}\left(y_{i}-y_{i}^{-}\right)=S\left(y_{i}\right)=-\left(y_{i} \times[-1,0]\right) \cup\left(y_{i} \times[-1,0] \subset A_{F}^{\prime}\right) \\
& \left.\left.\partial_{M V}^{-1}(m)=S_{A}(m)=D_{m}-(\Sigma \backslash(]-2,0] \times K\right)\right) \cup\left(-D_{m} \subset A_{F}^{\prime}\right)
\end{aligned}
$$


Since $x_{i}$ intersects only $y_{i}, S\left(x_{i}\right)$ intersects only $S\left(y_{i}\right)$ and $S_{A}(m)$. The algebraic intersection of $S\left(x_{i}\right), S\left(y_{i}\right)$ and $S_{A}(m)$ is -1 .

For the surgery $\left.\left.\left(B_{F}, B_{F}^{\prime}\right), S_{B}(m)=D_{m}+\Sigma \backslash(]-2,0\right] \times K\right) \cup\left(-D_{m} \subset B_{F}^{\prime}\right)$, and the algebraic intersection of $S\left(x_{i}\right), S\left(y_{i}\right)$ and $S_{B}(m)$ is 1 .

\subsection{Proof of Theorem 4.1}

Remark 9.4 For this proof, I could also have used the strategy of Section 11. But I prefer this self-contained proof.

First recall the following easy lemma that will be used several times.

Lemma 9.5 The variation of the linking number of two knots $J$ and $K$ after a $p / q-$ surgery on a knot $V$ in a rational homology sphere $M$ is given by the following formula:

$$
\operatorname{lk}_{M_{(V ; p / q)}}(J, K)=\operatorname{lk}_{M}(J, K)-\frac{q}{p} \operatorname{lk}_{M}(V, J) \operatorname{lk}_{M}(V, K) .
$$

Let $\left(K_{1}, K_{2}, \ldots, K_{n}\right)$ be a link where all the $K_{i}$ bound disjoint oriented surfaces $\Sigma^{i}$. Consider an embedding of $\coprod_{i=1}^{r} \Sigma^{i} \times[-1,2]$. Let $N=\{1,2, \ldots, n\}$. For $i \in N$, associate surfaces $F^{i}=\Sigma^{i} \backslash d^{i}$ and LP-surgeries $\left(A_{i}, A_{i}^{\prime}\right)=\left(A_{F^{i}}, A_{F^{i}}^{\prime}\right)$ and $\left(B_{i}, B_{i}^{\prime}\right)=\left(B_{F^{i}}, B_{F^{i}}^{\prime}\right)$ as in Section 9.1. Let $U_{i}$ be a meridian of $K_{i}$ passing through $d^{i} \times[-1,2]$, so that performing one of the two surgeries transforms $U_{i}$ into $\pm K_{i}$ and performing both or none of them leaves $U_{i}$ unchanged. Then

$$
\begin{aligned}
{\left[M ;\left(K_{i} ; p_{i} / q_{i}\right)\right] } & =M_{\left(U_{i} ; p_{i} / q_{i}\right)}-M_{\left(K_{i} ; p_{i} / q_{i}\right)} \\
& =\frac{1}{2}\left[M_{\left(U_{i} ; p_{i} / q_{i}\right)} ;\left(A_{i}, A_{i}^{\prime}\right),\left(B_{i}, B_{i}^{\prime}\right)\right] .
\end{aligned}
$$

More generally, for $J \subset\left\{\left(A_{i}, A_{i}^{\prime}\right),\left(B_{i}, B_{i}^{\prime}\right)\right\}_{i=1, \ldots, n}$,

$$
\left(M_{\left(U_{i} ; p_{i} / q_{i}\right)_{i \in N}}\right)_{J}=M_{\left(K_{i} ; p_{i} / q_{i}\right)_{i \in I(J)} \sharp \sharp j \notin I(J)} L\left(p_{j},-q_{j}\right)=M_{I(J)}
$$

where $I(J)$ is the set of elements $i$ of $N$ such that $\sharp\left(J \cap\left\{\left(A_{i}, A_{i}^{\prime}\right),\left(B_{i}, B_{i}^{\prime}\right)\right\}\right)$ is one. Note that $(-1)^{\sharp J}=(-1)^{\sharp I(J)}$ and that for any subset $I$ of $N$ there are $2^{n}$ subsets $J$ of the set of LP-surgeries such that $I(J)=I$. Thus

$$
\left[M ;\left(K_{i} ; p_{i} / q_{i}\right)_{i \in N}\right]=\frac{1}{2^{n}}\left[M_{\left(U_{i} ; p_{i} / q_{i}\right)_{i \in N}} ;\left(A_{i}, A_{i}^{\prime}\right)_{i \in N},\left(B_{i}, B_{i}^{\prime}\right)_{i \in N}\right] .
$$

In particular, we can apply Theorem 3.1 to compute $Z_{n}\left(\left[M ;\left(K_{i} ; p_{i} / q_{i}\right)_{i \in N}\right]\right)$. 
According to Lemma 9.3, the tripods associated to the surgery $\left(A_{F^{i}}, A_{F^{i}}^{\prime}\right)$ and to the surgery $\left(B_{F^{i}}, B_{F^{i}}^{\prime}\right)$ are

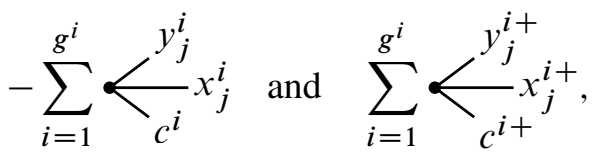

respectively. The only curve that links $c^{i}$ algebraically in $M_{\left(U_{i} ; p_{i} / q_{i}\right)_{i \in N}}$ among those appearing in all the tripods is $c^{i+}$ with a linking number $-q_{i} / p_{i}$. Therefore, these two must be paired together with this coefficient. Theorem 4.1 follows when $r=n$. The case $r>n$ can be either deduced from the case $r=n$ or proved directly, it is easy.

\section{Some clasper calculus}

The proofs of Theorem 4.3 and Theorem 4.4 will be given in Section 11. They will rely on the current section, where we recall some known clasper calculus and where we show how to present algebraically split links $L=\left(K_{i}\right)_{i=1, \ldots, n}$ by claspers so that the associated Seifert surfaces $\Sigma_{i}$ of the components $K_{i}$ in $M \backslash\left(\bigcup_{j \neq i} K_{j}\right)$ have minimal triple intersection, namely so that for any triple $\left(K_{i}, K_{j}, K_{k}\right)$ of components of $L$, the geometric triple intersection of the transverse surfaces $\Sigma_{i}, \Sigma_{j}$ and $\Sigma_{k}$ is made of $\left|\mu\left(K_{i}, K_{j}, K_{k}\right)\right|$ points. (This shows Proposition 4.5 that will be a direct corollary of Lemma 10.7 and Proposition 10.8.)

\subsection{Two ways of seeing surgeries on $Y$-graphs}

Let $\Lambda$ be the graph embedded in the surface $\Sigma(\Lambda)$ shown in Figure 6. In the 3handlebody $(N=\Sigma(\Lambda) \times[-1,1])$, the edges of $\Lambda$ are framed by a vector field normal to $\Sigma(\Lambda)=\Sigma(\Lambda) \times\{0\} . \Sigma(\Lambda)$ is called a framing surface for $\Lambda$.

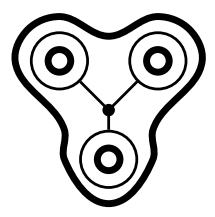

Figure 6: The graph $\Lambda$ in the surface $\Sigma(\Lambda)$

A $Y$-graph in $M$ is the isotopy class of an embedding $\phi$ of $N$ (or $\Sigma(\Lambda))$ into $M$. Such an isotopy class is determined by the framed image of the framed unoriented graph $\Lambda$ under $\phi$. A leaf of a $Y$-graph $\phi$ is the image under $\phi$ of a simple loop of our 
graph $\Lambda$. An edge of $\phi$ is an edge of $\phi(\Lambda)$ that is not a leaf. With this terminology, a $Y$-graph has three edges and three leaves. See Figure 7.

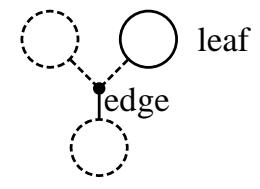

Figure 7: A $Y$-graph

The surgery on such a $Y$-graph can be defined in several equivalent ways.

Originally, it was defined by Matveev in [30] and named Borromeo transformation as the effect of the surgery on the 6-component framed link in the framed neighborhood of the $Y$-graph shown in Figure 8.

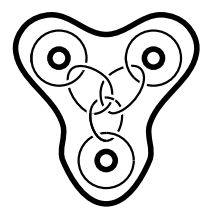

Figure 8: The surgery 6-component link

The framing of the link is induced by the framing of the surface.

We shall prove the following proposition.

Proposition 10.1 The above surgery is equivalent to the surgery $\left(A_{F}, A_{F}^{\prime}\right)$ associated with the subsurface $F$ of $\Sigma(\Lambda) \times[-1,1]$ shown in Figure 9, with respect to the notation of Section 9.1.
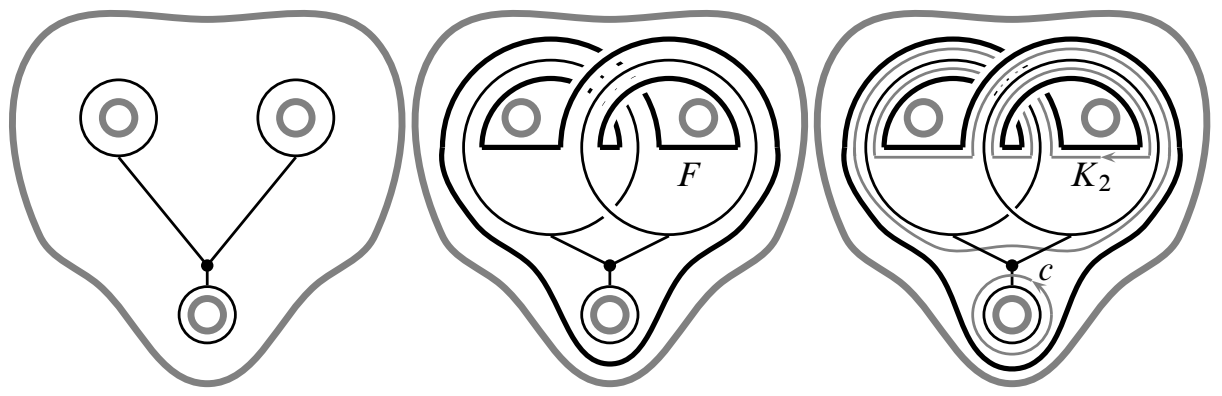

Figure 9: The surgery $\left(A_{F}, A_{F}^{\prime}\right)$ associated with a $Y$-graph 
Let $G \subset M$ be a $Y$-graph. A leaf $l$ of a $Y$-component of $G$ is trivial if $l$ bounds an embedded disc that induces the framing of $l$, in $M \backslash G$. It is easy to see that with both definitions, performing the surgery on a $Y$-graph with a trivial leaf does not change the ambient manifold. More precisely, the following lemma is proved in [14] by Garoufalidis, Goussarov and Polyak, for the first definition.

Lemma 10.2 [14, Lemma 2.1] Let $M$ be an oriented 3-manifold (with possible boundary). Let $G$ be a $Y$-graph in $M$ with a trivial leaf that bounds a disc $D$ in $M \backslash G$. Then

- for any framed graph $T_{0}$ in $M \backslash G$ that does not meet $D$, the pair $\left(M_{G}, T_{0}\right)$ is diffeomorphic to the pair $\left(M, T_{0}\right)$,

- if $T$ is a framed graph in $M \backslash G$ that meets $\operatorname{Int}(D)$ at exactly one point, then the pair $\left(M_{G}, T\right)$ is diffeomorphic to the pair $\left(M, T_{G}\right)$, where $T_{G}$ is the framed graph in $M$ shown in Figure 10.
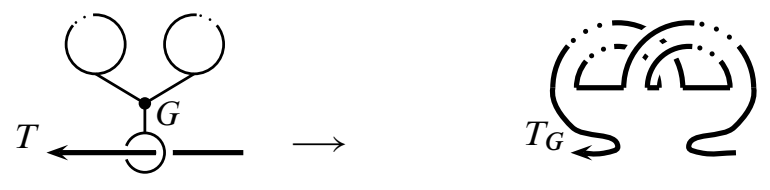

Figure 10

Now, it is proved in [3, Proof of Lemma 4.6], that this property fully determines the surgery. Therefore, since this property is also true for the second definition, the two definitions coincide and Proposition 10.1 is proved. In particular, the second definition has the same symmetries as the first one obviously has.

This definition does not depend on the orientation of $\Sigma(\Lambda)$. Nevertheless, we shall sometimes need orientations of our $Y$-graphs. An orientation of a $Y$-graph is an orientation of its three leaves, together with a cyclic order on the 3-element set they form, induced by an orientation of $\Sigma(\Lambda)$ as in Figure 11 (everything turns counterclockwise).

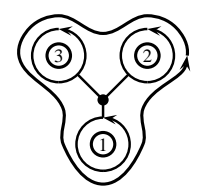

Figure 11 
An $n$-component $Y$-link $G \subset M$ is an embedding of the disjoint union of $n$ copies of $N$ into $M$ up to isotopy. The $Y$-surgery along a $Y$-link $G$ is defined as the surgery along each $Y$-component of $G$. The resulting manifold is denoted by $M_{G}$.

\subsection{Some clasper calculus}

Recall the following equivalences between surgeries inside handlebodies-that can be themselves embedded in any 3-manifold in an arbitrary way. The first one is move $Y_{3}$ in [14], as rectified by Emmanuel Auclair in his thesis [2].

Lemma 10.3 [2] The surgeries on the two $Y$-links of Figure 12 are equivalent.
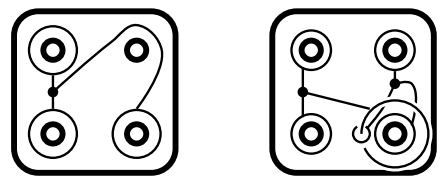

Figure 12

Lemma 10.4 [14, Theorem 3.1, move $\left.Y_{4}\right]$ The surgeries on the two $Y$-links of Figure 13 are equivalent.

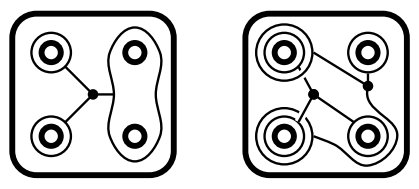

Figure 13

The two equivalences above easily imply the following one.

Lemma 10.5 The surgeries on the two $Y$-links of Figure 14 are equivalent.
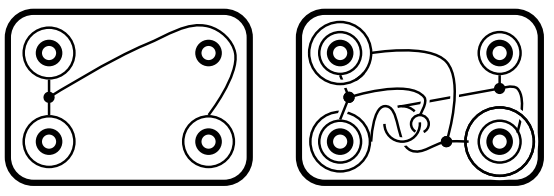

Figure 14

As a consequence of Lemma 10.4, we also have the following lemma that yields an inverse for a $Y$-graph. A mark $\%$ on an edge indicates a positive half-twist of this edge. 
Lemma 10.6 [14, Theorem 3.2] The surgery on the $Y$-link of Figure 15 is trivial.

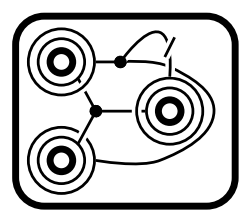

Figure 15

\subsection{A clasper presentation of algebraically split links}

A leaf $\ell$ of a $Y$-link $G$ is a meridional leaf or is a meridian of a link $L$, if it is trivial, and if it bounds a meridian disk of some link component whose interior intersects $G \cup L$ at exactly one point of $L$.

Say that a $Y$-link $G$ laces the trivial $r$-component link $U^{(r)}$ of a connected 3manifold if

- each of the $Y$-link components contains a meridional leaf of $U^{(r)}$,

- the components $U_{i}$ of $U^{(r)}$ bound disjoint disks $\left(D_{i}\right)_{i=1, \ldots, r}\left(U_{i}=\partial D_{i}\right)$ so that $D_{i} \cap G$ is inside the meridional leaves of $U_{i}$ (and contains one point per meridional leaf of $U_{i}$ ),

- no component of $G$ contains more than one meridional leaf of a given component $U_{i}$.

Performing the surgery on such a $G$ transforms $U^{(r)}$ into the link $\left(K_{1}, \ldots, K_{r}\right)=U_{G}^{(r)}$ in $M$ that is presented by $\left(G, U^{(r)}\right)$.

Since any null-homologous knot bounds an oriented Seifert surface, by Lemma 10.2, it is easy to see that any null-homologous knot is presented by a pair $\left(G, U_{1}\right)$, where $G$ is a $Y$-link that laces the trivial knot. See Figure 16.

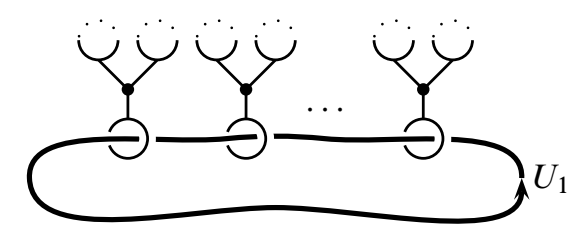

Figure 16: A $Y$-link that laces the trivial knot $U_{1}$ 
In a connected oriented compact 3-manifold $M$ such that $H_{2}(M ; \mathbb{Z})=0$, the linking number of a null-homologous knot $K$ with a knot $C$ in its complement is well-defined as the algebraic intersection of $C$ with a surface bounded by $K$. The Milnor triple linking number $\mu\left(K_{1}, K_{2}, K_{3}\right)$ of three null-homologous knots $K_{1}, K_{2}, K_{3}$ that do not link each other is also well-defined, as the following algebraic intersection of three Seifert surfaces of these knots in the complement of the other ones:

$$
\mu\left(K_{1}, K_{2}, K_{3}\right)=-\left\langle\Sigma_{1}, \Sigma_{2}, \Sigma_{3}\right\rangle
$$

Let $G$ be a $Y$-link that laces the trivial link $U^{(r)}$ of $M$. Let $m_{i}$ denote the homology class of the oriented meridian of $U_{i}$. Say that a component of $G$ is of type $\left(\varepsilon_{i} m_{i}, \varepsilon_{j} m_{j}, f\right)$ if its leaves are one meridian of $U_{i}$, one meridian of $U_{j}$, and another oriented framed leaf $f$ and if it can be oriented so that the homology classes of its oriented leaves read $\varepsilon_{i} m_{i}, \varepsilon_{j} m_{j}$ and $[f]$ with respect to the cyclic order induced by the orientation, with $\varepsilon_{i}, \varepsilon_{j} \in\{-1,1\}$. Similarly, say that a component of $G$ is of type $\left(\varepsilon_{i} m_{i}, \varepsilon_{j} m_{j}, \varepsilon_{k} m_{k}\right)$ if its leaves are one meridian of $U_{i}$, one meridian of $U_{j}$, and one meridian of $U_{k}$, and if it can be oriented so that the homology classes of its oriented leaves read $\varepsilon_{i} m_{i}, \varepsilon_{j} m_{j}$ and $\varepsilon_{k} m_{k}$ with respect to the cyclic order induced by the orientation, with $\varepsilon_{i}, \varepsilon_{j}, \varepsilon_{k} \in\{-1,1\}$.

Lemma 10.7 Let $G$ be a $Y$-link that laces the trivial link $U^{(r)}$ of an oriented connected 3-manifold $M$. Let $L=\left(K_{1}, \ldots, K_{r}\right)=U_{G}^{(r)}$ be the link presented by $G$. Then $L$ is algebraically split, and the $K_{i}$ bound surfaces $\Sigma_{i}$ such that

- for any $\{i, j\} \subset\{1,2, \ldots, r\}, \Sigma_{i} \cap \Sigma_{j}$ is the union over all the components of type $\left(\varepsilon_{i} m_{i}, \varepsilon_{j} m_{j}, f\right)$ of the framed oriented leaves $\varepsilon_{i} \varepsilon_{j} f$,

- for any $\{i, j, k\} \subset\{1,2, \ldots, r\}$, the oriented intersection $\Sigma_{i} \cap \Sigma_{j} \cap \Sigma_{k}$ is a union over all the components of type $\left(\varepsilon_{i} m_{i}, \varepsilon_{j} m_{j}, \varepsilon_{k} m_{k}\right)$ of points with sign $\varepsilon_{i} \varepsilon_{j} \varepsilon_{k}$.

In particular, if $H_{2}(M ; \mathbb{Z})=0$, then $\mu\left(K_{i}, K_{j}, K_{k}\right)$ is the sum over all the components of type $\left(\varepsilon_{i} m_{i}, \varepsilon_{j} m_{j}, \varepsilon_{k} m_{k}\right)$ of the contributions $\left(-\varepsilon_{i} \varepsilon_{j} \varepsilon_{k}\right)$.

Proof Define the index of a component $Y$ of $G$ as the smallest $i$ such that $Y$ has a meridional leaf of $U_{i}$. Realize the surgeries on the components of index $i$ of $G$ by applying Lemma 10.2 to the trivial meridional leaf $\ell$ of $U_{i}$ and to the part of $U_{i}$ passing through $\ell$. These surgeries transform $U^{(r)}$ into $L$ and allow us to see each $K_{i}$ as the boundary of a surface $\widetilde{\Sigma}_{i}$ whose 1 -handles are thickenings of the framed leaves that are not meridians of $U_{i}$ of the components of index $i$.

So far, $\widetilde{\Sigma}_{k}$ may intersect the $K_{i}$ with $i<k$ (but not the $K_{i}$ with $i>k$ ). More precisely, if $i<k$, each component of index $i$ of type $\left(m_{i}, \varepsilon m_{k}, f\right)$ or $\left(m_{i}, f,-\varepsilon m_{k}\right)$ gives rise 
to an arc of intersection of $\widetilde{\Sigma}_{i} \cap \widetilde{\Sigma}_{k}$. Tubing $\widetilde{\Sigma}_{k}$ along the part of $K_{i}$ between the two extremities of the intersection arc that is contained in the surgery picture transforms this arc of intersection into $\varepsilon f$ and removes the intersection of $K_{i}$ with $\widetilde{\Sigma}_{k}$. See Figure 17 .
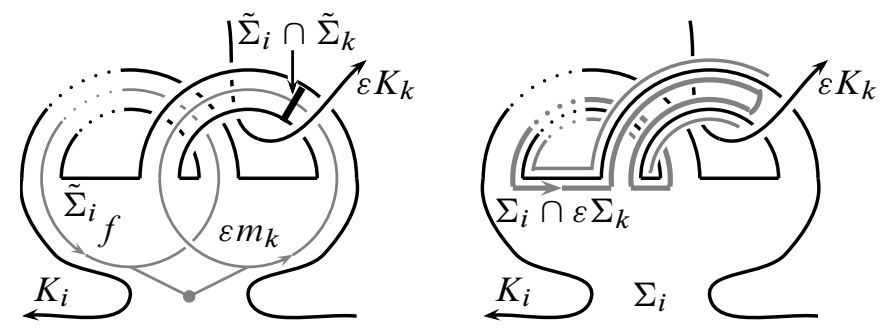

Figure 17

If the third leaf is a meridian of $K_{j}$ for $i<j<k$ then perform the tubing along this leaf inside the tubing of $\widetilde{\Sigma}_{j}$ along the meridional leaf of $m_{k}$. Let $\Sigma_{k}$ denote the surface obtained after all these tubings. See Figure 18.

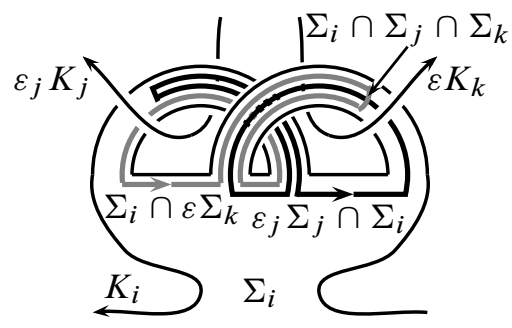

Figure 18

It is left to the reader to check that the surfaces have the announced properties.

Say that a $Y$-link $G \mu$-laces the trivial $r$-component link $U^{(r)}$ of a connected 3manifold if it laces it, and if for any triple $\{i, j, k\}$ of integers in $\{1, \ldots, r\}$, there are exactly $\left|\mu\left(K_{i}, K_{j}, K_{k}\right)\right|$ components with one leaf that links $U_{i}$, one leaf that links $U_{j}$ and one leaf that links $U_{k}$.

It is known that any algebraically split link can be presented by a $Y$-link $G$ that laces the trivial link $U^{(r)}$ by [14, Lemma 5.6], Matveev [30] or Murakami and Nakanishi [32]. We prove the following proposition that refines this result, and that, together with Lemma 10.7, proves Proposition 4.5. 
Proposition 10.8 For any algebraically split link $L=\left(K_{1}, \ldots, K_{r}\right)$ in a connected 3-manifold $M$ such that $H_{2}(M ; \mathbb{Z})=0$, there exists a $Y$-link $G$ that $\mu$-laces the trivial link $U^{(r)}$ of $M$ such that $\left(G, U^{(r)}\right)$ presents $L$.

This proposition will be a direct corollary of the slightly more general Proposition 10.9 below, that may be used for the study of homology handlebodies.

Here, an $r$-component based link is an embedding $\Gamma_{L}$ of the graph with $r$ loops depicted in Figure 19, up to isotopy. Its underlying link is the restriction of the embedding to its $r$ loops.

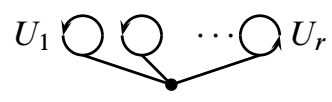

Figure 19

The trivial $r$-component based link $\Gamma_{U}^{(r)}$ is the $r$-component based link of Figure 19 seen in a $3-$ ball.

Proposition 10.9 For any based $r$-component link $\Gamma_{L}$ whose underlying link $L=$ $\left(K_{1}, \ldots, K_{r}\right)$ is algebraically split in a connected 3-manifold $M$ with $H_{2}(M ; \mathbb{Z})=0$, there exists a $Y$-link $G$ in $M \backslash \Gamma_{U}^{(r)}$ that $\mu$-laces the trivial link $U^{(r)}$ of $M$ such that $\left(G, \Gamma_{U}^{(r)}\right)$ presents $\Gamma_{L}$.

Proof For any sublink $L^{\prime}$ of $L$, there is a canonical subgraph $\Gamma_{L^{\prime}}$ of $\Gamma_{L}$ that is a based link with underlying link $L^{\prime}$. We prove Proposition 10.9 by proving the following statement by induction on the number $r$ of components of $L$.

Induction hypothesis Let $M$ be a connected 3-manifold such that $H_{2}(M ; \mathbb{Z})=0$. Let $\Gamma_{L \cup L^{\prime}}$ be a based algebraically split link in $M$ where $L$ has $r$ components. Let $\Gamma_{U^{(r)} \cup L^{\prime}}$ be the based link obtained from $\Gamma_{L \cup L^{\prime}}$ by replacing $\Gamma_{L}$ by $\Gamma_{U}^{(r)}$ so that each component of $U^{(r)}$ bounds a disk $D_{i}$ whose interior does not meet $\Gamma_{U^{(r)} \cup L^{\prime}}$. Then there exists a $Y$-link $G$ in $M \backslash \Gamma_{L^{\prime}}$ such that the following set of properties $H\left(G, \Gamma_{L}, \Gamma_{L^{\prime}}\right)$ is satisfied:

- $G \subset M \backslash \Gamma_{U^{(r)} \cup L^{\prime}}$.

- $G \mu$-laces the trivial link $U^{(r)}$ of $M \backslash L^{\prime}$.

- $\left(G, \Gamma_{U^{(r)} \cup L^{\prime}}\right)$ presents $\Gamma_{L \cup L^{\prime}}$ in $M$.

- The only leaves of $G$ that link $L^{\prime}$ algebraically are meridional leaves of $L^{\prime}$. 
- No component of $G$ contains more than one meridional leaf of a given component of $L^{\prime}$.

- For any triple $\{I, J, K\}$ of components of $L \cup L^{\prime}$ with at least one component in $L$, there are exactly $|\mu(I, J, K)|$ components of $G$ with one leaf that links $I$, one leaf that links $J$ and one leaf that links $K$.

This statement is obviously true for 0 -component links.

Assume that it is true for $(r-1)$-component links, we wish to prove it for ( $L=$ $\left.\left(K_{1}, \ldots, K_{r}\right), L^{\prime}\right)$. Let $U^{(r-1)}=\left(U_{1}, \ldots, U_{r-1}\right)$ denote the trivial $(r-1)$-component link that bounds a disjoint union of disks $\left(D_{i}\right)_{i=1, \ldots, r-1}$. By induction, there exists $\left(G_{1} \subset M \backslash \Gamma_{L^{\prime} \cup K_{r}}\right)$ such that $H\left(G_{1}, \Gamma_{K_{1}, \ldots, K_{r-1}}, \Gamma_{K_{r} \cup L^{\prime}}\right)$ is satisfied.

Consider a two-dimensional disk $D$ that meets $K_{r}$ along an arc $\alpha$ of its boundary around which all the meridional leaves of $K_{r}$ are, and such that $D$ intersects all the meridional leaves, so that

$$
K_{r}^{\prime}=\left(K_{r} \backslash \stackrel{\circ}{\alpha}\right) \cup(-\partial D \backslash \alpha)
$$

bounds a surface $\Sigma$ that meets neither $\Gamma_{L^{\prime} \cup U^{r-1}} \cup \bigcup_{i<r} D_{i}$, nor the path $\gamma_{r}$ from the vertex of $\Gamma_{L \cup L^{\prime}}$ to $K_{r}$, nor the leaves of $G_{1}$. See Figure 20.

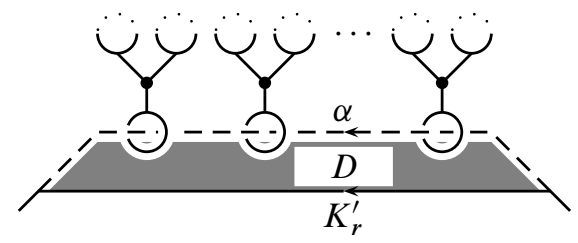

Figure 20

Lemma 10.10 The graph $G_{1}$ and the surface $\Sigma$ can be modified so that $\Sigma$ does not meet $G_{1}$ at all outside the meridional leaves of $L$, and the following set of assumptions $H_{2}\left(G_{1}, \Gamma_{K_{1}, \ldots, K_{r-1}}, \Gamma_{K_{r} \cup L^{\prime}}, \Sigma\right)$ is still satisfied.

- $\Sigma$ meets neither $\Gamma_{U^{(r-1)} \cup L^{\prime}} \cup \bigcup_{i<r} D_{i}$, nor $\gamma_{r}$, nor the leaves of $G_{1}$.

- $H\left(G_{1}, \Gamma_{K_{1}, \ldots, K_{r-1}}, \Gamma_{K_{r} \cup L^{\prime}}\right)$ is satisfied except that components of $G_{1}$ are allowed to have no meridians of $L \backslash K_{r}$ provided that they have a meridian of $K_{r}$.

Proof We need to remove the intersections of $\Sigma$ with the edges of $G_{1}$. By isotopy, without loss, assume that no edge adjacent to a meridional leaf of $K_{r}$ intersects $\Sigma$ 
(push the intersection on the two other edges, if necessary). Similarly, assume that if a component contains only one meridian of $L$, the edge adjacent to this component does not meet $\Sigma$. Now, the intersections of the edges adjacent to nonmeridional leaves can be removed by tubing $\Sigma$ around the part of the $Y$-graph that contains the corresponding leaf. Here tubing means replacing a small disk of $\Sigma$ in a neighborhood of an intersection point with an edge by the closure of its complement in the boundary of a regular neighborhood of the part of the $Y$-graph that contains the corresponding leaf, as in Figure 21.
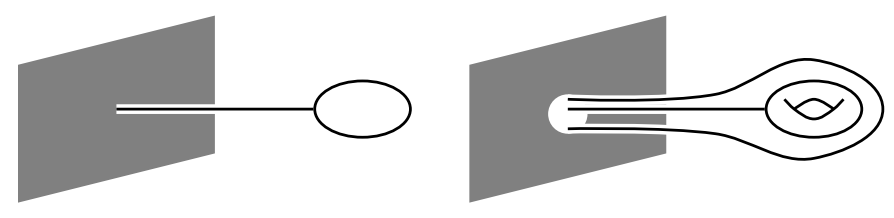

Figure 21

Thus, we are under the assumptions $H(E)$ that the only edge intersections occur on edges adjacent to a meridional leaf of some $K_{j}$, with $j<r$, of components with at least two meridional leaves of $L$. Define the complexity $c\left(\Sigma ; G_{1}\right)$ of such a situation as follows. Define the complexity $c_{e}(Y)$ of a component $Y$ of $G_{1}$ as the number of intersection points of its edges with $\Sigma$. Define the complexity $c\left(\Sigma ; G_{1}\right)$ as the pair (maximal complexity $c_{e}$ of the components, number of components with this complexity) ordered by the lexicographic order.

Now, to prove the lemma, it is enough to prove that there exists a pair $\left(\Sigma ; G_{1}\right)$ with lower complexity such that $H_{2}\left(G_{1}, \Gamma_{K_{1}, \ldots, K_{r-1}}, \Gamma_{K_{r} \cup L^{\prime}}, \Sigma\right)$ and $H(E)$ are satisfied.

Consider a component $Y$ of $G_{1}$ with maximal complexity, and its edge $e$ with the maximal number of intersection points. By hypothesis, $e$ is adjacent with a meridional leaf $\ell$ of some component $K_{i}$ with $i<r$. Remove the point of $e \cap \Sigma$ that is closest to $\ell$ as follows. By our assumptions, $\Sigma$ intersects a neighborhood of $Y$ in the gray part of Figure 22, where the intersection point that will be removed is at the top right corner. Perform the modification of Lemma 10.5 so that the resulting three graphs are like in Figure 22 with respect to the positions of the possible intersections with $\Sigma$. Let $Y_{1}$ be the graph that replaces $Y$ with one edge intersection removed. Let $Y_{3}$ be the graph with a meridional leaf of $K_{r}$, a leaf parallel to $\ell$, and another trivial leaf $\ell_{1}$, and let $Y_{2}$ be the other one with one meridian of $\ell_{1}$. Remove all the intersection of $Y_{3}$ with $\Sigma$ outside its meridional leaf of $K_{r}$ by tubing. If $Y_{2}$ has only one meridional leaf of $L$, then remove its intersections as before, too. Otherwise, don't change it, it has two meridional leaves, and its complexity $c_{e}$ is lower than $c_{e}(Y)$. Slide the meridional leaf 

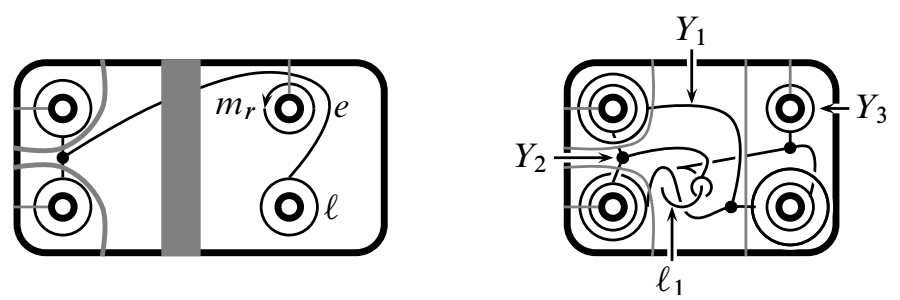

Figure 22

of $K_{r}$ in $Y_{3}$ so that it is around the arc $\alpha$ of $K_{r}$. Thus, the obtained graph and the modified $\Sigma$ together satisfy $H_{2}\left(G_{1}, \Gamma_{K_{1}, \ldots, K_{r-1}}, \Gamma_{K_{r} \cup L^{\prime}}, \Sigma\right)$ and $H(E)$, and have lower complexity. The lemma is proved.

By Lemma 10.2, $K_{r} \backslash \alpha$ is obtained from $\partial D \backslash \alpha$ by surgery on a $Y$-link $G_{2}$ in the neighborhood of $\Sigma \backslash D$ such that any component of $G_{2}$ contains exactly one meridian of $\partial D$. Let $U_{r}=\partial D$. Thus, $K_{r}$ is obtained from $U_{r}$ by surgery on $G_{1} \cup G_{2}, G_{1} \cup G_{2}$ $\mu$-laces the trivial link $U^{(r)}$ of $M \backslash L^{\prime},\left(G_{1} \cup G_{2}, \Gamma_{U^{(r)} \cup L^{\prime}}\right)$ presents $\Gamma_{L \cup L^{\prime}}$ in $M$. Let us now modify $G=G_{1} \cup G_{2}$ so that the last three conditions of $H\left(G, \Gamma_{L}, \Gamma_{L^{\prime}}\right)$ are satisfied in addition to the previous ones.

- Cutting the leaves so that the only leaves that link $L^{\prime}$ algebraically are meridional leaves of $L^{\prime}$

Use move $Y_{4}$ of [14] (Lemma 10.4) to cut the leaves of $G_{2}$ that are not meridians of $K_{r}$ so that they are either 0 -framed meridians of $L^{\prime}$ or they do not link $L^{\prime}$ at all. Indeed, this move allows us to cut the leaves into leaves that are homologically trivial in the complement of $L^{\prime}$, and meridians of the components of $L^{\prime}$ without creating further intersections of $G_{2}$ with the disk $D$. Define the complexity of a leaf as the minimal number of leaves in such a decomposition minus one. Define the complexity of a $Y$-graph as the sum of the complexities of its leaves. Finally define the complexity of a $Y$-link as the pair (maximal complexity of the components, number of components with this complexity) ordered by the lexicographic order. The leaves can be cut in order to make this complexity decrease without creating further intersections of $G$ with $D$.

- Sliding the handles so that no component of $G$ contains more than one meridional leaf of a given component of $L^{\prime}$.

Now, we wish to remove the $Y$-components with a meridional leaf of $K_{r}$ and two meridional leaves of the same component $J$ of $L^{\prime}$. By Lemma 10.2, a surgery with respect to such a graph $G_{3}$ corresponds to a band sum with the boundary of a genus one Seifert surface as in Figure 23 where $\alpha$ and $\beta$ are meridians of $J$. 


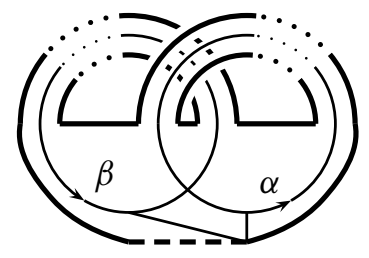

Figure 23

In this figure, a right-handed (resp. left-handed) Dehn twist of the surface along the simple curve $c(\alpha)$ freely homotopic to $\alpha$ transforms $\beta$ into $\alpha \beta$ (resp. $\alpha^{-1} \beta$ ) and does not change $\alpha$. Therefore, the $Y$-graph $G_{3}$ is equivalent to a $Y$-graph whose leaves are a meridian of $K_{r}$, the meridian $\alpha$, and the curve among $\alpha \beta$ and $\alpha^{-1} \beta$ that is null-homologous.

- Realizing the algebraic cancellations to the Milnor invariants $\mu\left(K_{r}, K_{s}, K_{t}\right)$ where $K_{s}$ and $K_{t}$ are components of $L^{\prime}$.

First recall from Lemma 10.7 that $\mu\left(K_{r}, K_{s}, K_{t}\right)$ is the sum of the contributions $\varepsilon \eta$ of the $Y$-graphs of type $\left(m_{r},-\varepsilon m_{s}, \eta m_{t}\right)$ or $\left(m_{r}, \varepsilon m_{t}, \eta m_{s}\right)$ where $\varepsilon$ and $\eta$ belong to $\{-1,1\}$. Second, exchange the order of the $Y$-graphs that link $U_{r}$ so that all the graphs that contribute with a sign opposite to the Milnor invariant are followed by a graph that contributes with the sign of the Milnor invariant. In order to exchange two $Y$-graphs that link $U_{r}$, perform the sequence of operations shown in Figure 24. First slide the meridian $m$ of one of them inside the other one $m^{\prime}$. Next use move $Y_{4}$
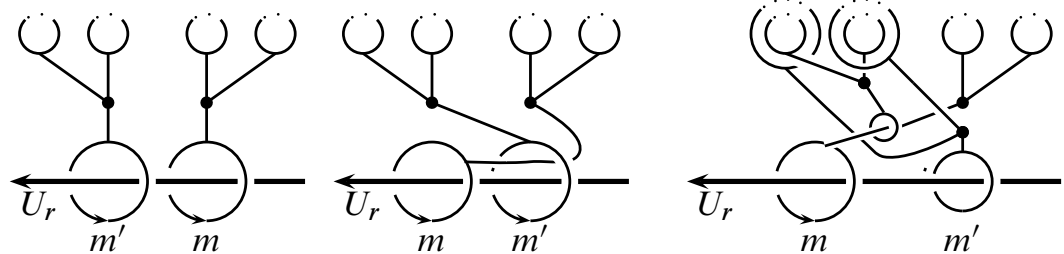

Figure 24

(Lemma 10.4) to cut $m^{\prime}$ into $m^{\prime}$ and a leaf that links the edge going to $m$. It is enough to slide inside components that contribute to $\mu\left(K_{r}, K_{s}, K_{t}\right)$. Thus, we do not lose properties of our graphs, (and otherwise we could just perform the surgery).

Last, transform a pair of $K_{r}$-adjacent $Y$-graphs with opposite contributions to the Milnor invariant $\mu\left(K_{r}, K_{s}, K_{t}\right)$ into a family of $Y$-graphs that do not individually contribute to $\mu\left(K_{r}, K_{S}, K_{t}\right)$. To do this, see the effect of the surgery along the two $K_{r}$-adjacent $Y$-graphs as a band sum with the boundary of a genus two surface $\Sigma$ 
whose 1-handles are $\alpha_{1}, \beta_{1}, \alpha_{2}, \beta_{2}$, and are meridians of $K_{s}$ and $K_{t}$. See Figure 25 .

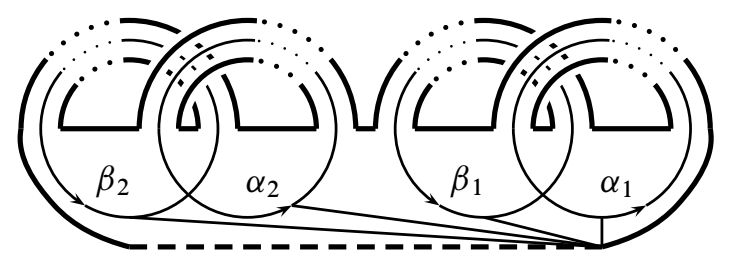

Figure 25

We are in one of the following situations for the homology classes of the curves: Either $\left[\alpha_{1}\right]=\left[\alpha_{2}\right]$ and $\left[\beta_{1}\right]=-\left[\beta_{2}\right]$, or $\left[\alpha_{1}\right]=-\left[\alpha_{2}\right]$ and $\left[\beta_{1}\right]=\left[\beta_{2}\right]$, or $\left[\alpha_{1}\right]=\left[\beta_{2}\right]$ and $\left[\beta_{1}\right]=\left[\alpha_{2}\right]$, or $\left[\alpha_{1}\right]=-\left[\beta_{2}\right]$ and $\left[\beta_{1}\right]=-\left[\alpha_{2}\right]$.

Consider the following simple closed curves $c\left(\alpha_{2}\right), c\left(\beta_{1}\right), c\left(\beta_{2}\right), c\left(\beta_{1} \alpha_{2}\right)$ and $c\left(\beta_{1} \beta_{2}\right)$ depicted in Figure 26 whose homology classes are $\left[\alpha_{2}\right],\left[\beta_{1}\right],\left[\beta_{2}\right],\left[\beta_{1} \alpha_{2}\right]$ and $\left[\beta_{1} \beta_{2}\right]$, respectively.

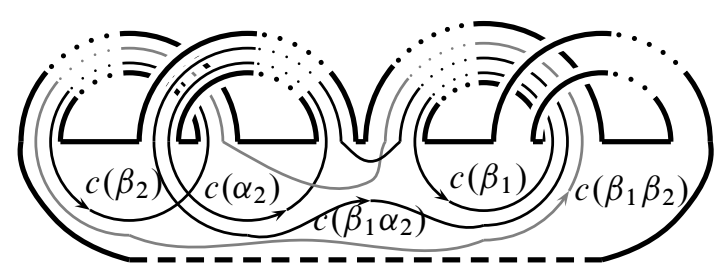

Figure 26

For a curve $c$, let $\tau_{c}$ denote the right-handed Dehn twist around this curve. Recall the action of $\tau$ on homology classes $\tau_{c}(x)=x+\langle c, x\rangle_{\Sigma} c$. Then the homeomorphism $\tau_{c\left(\alpha_{2}\right)}^{-1} \tau_{c\left(\beta_{1}\right)}^{-1} \tau_{c\left(\beta_{1} \alpha_{2}\right)}$ of $\Sigma$ transforms $\alpha_{2}$ and $\beta_{1}$ to conjugate curves, where the conjugation paths are in the neighborhood of the genus 2 surface and avoids the disks $D_{i}$, for $i \leq r$, and it transforms $\alpha_{1}$ and $\beta_{2}$ into curves homologous to $\alpha_{1} \alpha_{2}^{-1}$ and $\beta_{1} \beta_{2}$. Therefore using this boundary-preserving homeomorphism in the first case allows us to transform the surgery on the initial pair of $Y$-graphs into a surgery on a pair of $Y-$ graphs such that each of the graphs has a homologically trivial leaf and two meridional

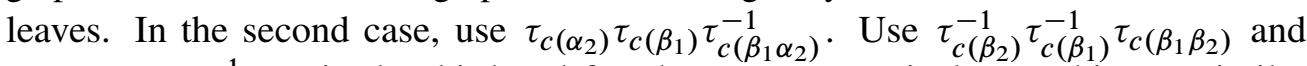

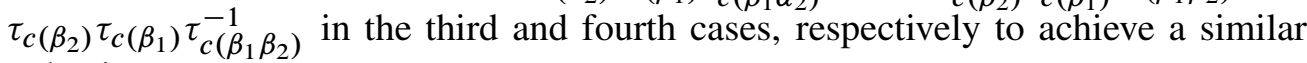
reduction. 


\section{Proof of the formulae for algebraically split links}

We prove the surgery formulae of Theorem 4.3 and Theorem 4.4 following a strategy that was used by Garoufalidis, Goussarov and Polyak in [14] to compare the filtration of the space of $\mathbb{Z}$-spheres associated with algebraically split links to the filtration associated with $Y$-links.

According to Proposition 10.8, it is enough to prove these theorems for links that are presented by pairs $\left(G, U^{(r)}\right)$ where $G$ is a $Y$-link that $\mu$-laces the trivial link $U^{(r)}$ of $M$, that is for $U^{(r)} \subset M_{G}$, where $U^{(r)}$ is equipped with surgery coefficients $p_{1} / q_{1}$, $p_{2} / q_{2}, \ldots, p_{r} / q_{r}$.

$$
\left[M_{G} ; U^{(r)}\right]=\sum_{H \subset G}(-1)^{\sharp H}\left[M ; H \cup U^{(r)}\right]
$$

where

$$
\left.\left[M ; H \cup U^{(r)}\right]=\sum_{\substack{J \subset H, I \subset\{1,2, \ldots, r\}}}(-1)^{\sharp J+\sharp I} M_{J,\left(U_{i} ; p_{i} / q_{i}\right)_{i \in I} \sharp(\sharp j \in\{1,2, \ldots, r\} \backslash I} L\left(p_{j},-q_{j}\right)\right) .
$$

Then $\left[M_{G} ; U^{(r)}\right] \equiv_{n} \sum_{H \subset G ; \# H \leq 2 n}(-1)^{\sharp H}\left[M ; H \cup U^{(r)}\right]$.

- If there exists $i$ such that $U_{i}$ does not link any leaf of $H$, then $\left[M ; H \cup U^{(r)}\right]=0$.

- If there exists $i$ such that $U_{i}$ links only one leaf of $H$, then letting $Y_{1}$ be the component of this leaf,

$$
\left[M ; H \cup U^{(r)}\right]=-\left[M_{Y_{1}} ; H \backslash Y_{1} \cup U^{(r)}\right] .
$$

Recall that the surgery on $Y_{1}$ is a surgery associated with a genus one surface bounded by some $K_{i}$ as in Section 9.1. Then the inverse surgery of this subsection transforms $U_{i}$ into $-K_{i}$ and since it can be realized as a genus one cobordism, it can also be realized by a surgery on a $Y$-graph that laces $U_{1}$ and that sits in the complement of $G$. Let $Y_{1}^{-1}$ be such a graph. We can assume that its leaves are a meridian of $U_{i}$ and two leaves parallel to the two other leaves of $Y_{1}$. Compare Lemma 10.6.

Then $\left[M_{Y_{1}} ; H \backslash Y_{1} \cup U^{(r)}\right]=\left[M_{Y_{1}^{-1}} ; H \backslash Y_{1} \cup U^{(r)}\right]$ and

$$
\left[M ; H \cup U^{(r)}\right]=\frac{1}{2}\left[M ; H \cup Y_{1}^{-1} \cup U^{(r)}\right]
$$


As long as there is a component $U_{j}$ that bounds a disk $D_{j}$ intersecting the union $H \cup Y_{1}^{-1} \cup \ldots \cup Y_{k}^{-1}$ once (and necessarily) inside a meridional leaf of some component $Y_{k+1}$ of $H$, add $Y_{k+1}^{-1}$, and write

$$
\begin{aligned}
{\left[M ; H \cup Y_{1}^{-1} \cup \cdots \cup Y_{k}^{-1} \cup U^{(r)}\right] } & =\frac{1}{2}\left[M ; H \cup Y_{1}^{-1} \cup \cdots \cup Y_{k}^{-1} \cup Y_{k+1}^{-1} \cup U^{(r)}\right], \\
{\left[M ; H \cup U^{(r)}\right] } & =\frac{1}{2^{k+1}}\left[M ; H \cup Y_{1}^{-1} \cup \cdots \cup Y_{k}^{-1} \cup Y_{k+1}^{-1} \cup U^{(r)}\right] .
\end{aligned}
$$

Finally, $\left[M_{G} ; U^{(r)}\right]$ is a rational combination of terms of the form $\left[M ; H^{\prime} \cup U^{(r)}\right]$ where each $U_{i}$ links at least two leaves of $H^{\prime}$. To be more specific, the considered $H^{\prime}$ are of the form $H \cup H_{1}^{-1}$, where $H$ is a sublink of $G$, and $H_{1}^{-1}$ is a link made of inverses of the components of a sublink $H_{1}$ of $H$. In particular, the leaves of a component of $H_{1}^{-1}$ have the same constraints as the leaves of a component of $G$. Since a leaf of $H^{\prime}$ links at most one $U_{i}$, such a $H^{\prime}$ has at least $2 r$ leaves linking the $U_{i}$. In particular, if $2 r>6 n,\left[M_{G} ; U^{(r)}\right] \equiv_{n} 0$.

- Under the hypotheses of Theorem 4.3, assume $2 r=6 n$. Up to elements in $\mathcal{F}_{2 n+1}$, $\left[M_{G} ; U^{(r)}\right]$ is a rational combination of terms of the form $\left[M ; H^{\prime} \cup U^{(r)}\right]$ where each $U_{i}$ links exactly two leaves of $H^{\prime}$, and each leaf of $H^{\prime}$ is a meridional leaf of some $U_{i}$. More precisely, let $G_{3}$ be the sublink of $G$ made of the components that have three meridional leaves, we have

$$
\left[M_{G} ; U^{(r)}\right] \equiv_{n} \sum_{H}(-1)^{\sharp H}\left[M ; H \cup U^{(r)}\right]
$$

where the sum runs over the $H$ that read as the disjoint union of two $Y$-links $H_{1}$ and $H_{2}$ of $G_{3}$ such that for any component $U_{i}$ of $U^{(r)}$, either there is one meridional leaf of $U_{i}$ in $H_{1}$ and no meridional leaf of $U_{i}$ in $H_{2}$, or there is no meridional leaf of $U_{i}$ in $H_{1}$ and there are two meridional leaves of $U_{i}$ in $H_{2}$. Let $\mathcal{H}$ denote the set of the $\left(H_{1}, H_{2}\right)$ where $H_{1} \cup H_{2}$ is a decomposition as above of such a graph.

- Under the hypotheses of Theorem 4.4, at most two thirds of the leaves of the $H^{\prime}$ link the $U_{i}$ once, and the leaves of the other third do not link the $U_{i}$ at all. Therefore, if $2 r>4 n,\left[M_{G} ; U^{(r)}\right]$ belongs to $\mathcal{F}_{2 n+1}$. If $r=2 n$, up to elements in $\mathcal{F}_{2 n+1}$, $\left[M_{G} ; U^{(r)}\right]$ is a rational combination of terms of the form $\left[M ; H^{\prime} \cup U^{(r)}\right]$ where each $U_{i}$ links exactly two leaves of $H^{\prime}$, and in each component of $H^{\prime}$, there are two meridional leaves of $U^{(r)}$ and a null-homologous leaf. More precisely, let $G_{2}$ be the sublink of $G$ made of the components that have two meridional leaves, we have

$$
\left[M_{G} ; U^{(r)}\right] \equiv_{n} \sum_{H}(-1)^{\sharp H}\left[M ; H \cup U^{(r)}\right]
$$


where the sum runs over the $H$ that read as the disjoint union of two $Y$-links $H_{1}$ and $H_{2}$ of $G_{2}$ such for any component $U_{i}$ of $U^{(r)}$, either there is one meridional leaf of $U_{i}$ in $H_{1}$ and no meridional leaf of $U_{i}$ in $H_{2}$, or there is no meridional leaf of $U_{i}$ in $H_{1}$ and there are two meridional leaves of $U_{i}$ in $H_{2}$. Let $\mathcal{H}$ denote the set of the $\left(H_{1}, H_{2}\right)$ where $H_{1} \cup H_{2}$ is a decomposition as above of such a graph.

In both cases

$$
\left[M_{G} ; U^{(r)}\right] \equiv_{n} \sum_{\left(H_{1}, H_{2}\right) \in \mathcal{H}}\left(\frac{-1}{2}\right)^{\sharp H_{1}}(-1)^{\sharp H_{2}}\left[M ; H_{1} \cup H_{1}^{-1} \cup H_{2} \cup U^{(r)}\right]
$$

where $H_{1} \cup H_{1}^{-1} \cup H_{2}$ has $2 n$ components (and therefore $(-1)^{\sharp H_{2}}=1$ ). Apply Theorem 3.1 to compute this. The tripod associated with a surgery on an oriented $Y-$ graph whose leaves are $\ell_{1}, \ell_{2}, \ell_{3}$ was computed in Lemma 9.3 (thanks to Proposition 10.1). It is

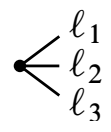

while the tripod associated with an inverse of such a graph is

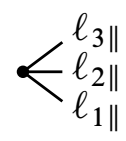

where the parallels are taken with respect of the parallelization of the leaves. Later, we shall consider twice the tripods of the components of $H_{1}$ and remove the $(-1)^{\sharp H_{1}}$. Recall the formula of Lemma 9.5:

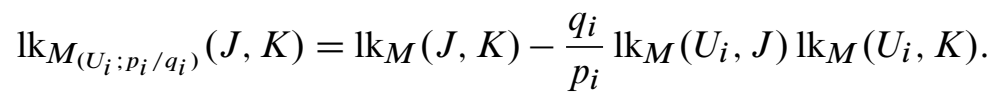

If for some $i$, a contraction does not pair two curves linking $U_{i}$, then its contribution to $\left[M ; H^{\prime} \cup\left(U \backslash U_{i}\right)\right]$ and its contribution to $\left[M_{U_{i}} ; H^{\prime} \cup\left(U \backslash U_{i}\right)\right]$ will be the same. Therefore, it won't contribute to $\left[M ; H^{\prime} \cup U\right]$. Thus since there are exactly two leaves $m_{i}$ and $m_{i}^{\prime}$ linking $U_{i}$ in each $H^{\prime}$, the only pairings that will contribute will pair these pairs together, and the corresponding remaining linking number will be $\left(q_{i} / p_{i}\right) \mathrm{lk}_{M}\left(U_{i}, m_{i}\right) \mathrm{lk}_{M}\left(U_{i}, m_{i}^{\prime}\right)$.

- Under the hypotheses of Theorem 4.3, there is one contributing pairing for every $\left(H_{1}, H_{2}\right) \in \mathcal{H}$. It can be seen as an edge-labelled Jacobi diagram $\Gamma\left(H_{1}, H_{2}\right)$ together with a bijection $b$ from the set of its vertices to the set of components of $H_{1} \cup H_{1}^{-1} \cup H_{2}$ that maps a vertex $v$ with adjacent edges labelled by $i, j, k$ to a component $b(v)$ of $G$ of type $\left(\varepsilon_{i} m_{i}, \varepsilon_{j} m_{j}, \varepsilon_{k} m_{k}\right)$ where $\varepsilon_{i}, \varepsilon_{j}, \varepsilon_{k}$ are in $\{-1,1\}$, or to the inverse of 
such a component. Equip $\Gamma\left(H_{1}, H_{2}\right)$ with an orientation. Then if the orientation of a vertex $v$ as above is induced by the cyclic order $(i, j, k)$, assign it the sign $\left(-\varepsilon_{i} \varepsilon_{j} \varepsilon_{k}\right)$, and assign it $\varepsilon_{i} \varepsilon_{j} \varepsilon_{k}$ otherwise. Define $\operatorname{sign}\left(\Gamma\left(H_{1}, H_{2}\right) ; b\right)$ as the products of the signs of the vertices. Then

$$
Z_{n}\left(\left[M_{G} ; U^{(r)}\right]\right)=\left(\prod_{i=1}^{3 n} \frac{q_{i}}{p_{i}}\right) \sum_{\left(H_{1}, H_{2}\right) \in \mathcal{H}} \frac{1}{2^{\sharp H_{1}}} \operatorname{sign}\left(\Gamma\left(H_{1}, H_{2}\right) ; b\right)\left[\Gamma\left(H_{1}, H_{2}\right)\right] .
$$

Now, let $f=f(b)$ be the map from $V\left(\Gamma\left(H_{1}, H_{2}\right)\right)$ to the set of components of $G$ obtained from a bijection $b$ as above by setting

$$
f(b)(v)= \begin{cases}b(v) & \text { if } b(v) \text { is a component of } H_{1} \cup H_{2}, \\ Y_{i} & \text { if } b(v)=Y_{i}^{-1} .\end{cases}
$$

There are $2^{\sharp H_{1}}$ bijections $b$ such that $f(b)=f$, and, if $\sharp \operatorname{Aut}_{e}(\Gamma)$ is the set of automorphisms of $\Gamma$ that induce the Identity on $E(\Gamma)$, there are $\sharp$ Aut $_{e}(\Gamma)$ bijections $b$ that define the same pairing. Since an automorphism that preserves the edges pointwise may only exchange vertices inside components $\Theta, \sharp$ Aut $_{e}(\Gamma)=2^{\theta(\Gamma)}$.

Orient $G$ arbitrarily. Let $\Gamma \in D_{e, n}$. Equip $\Gamma$ with an arbitrary orientation. Let $G(\Gamma)$ be the set of maps $g$ from $V(\Gamma)$ to the set of components of $G$ that map a vertex $v$ with adjacent edges labelled by $i, j, k$, with respect to the order induced by the orientation, to a component $g(v)$ of $G$ of type $\left(\varepsilon_{i} m_{i},-\varepsilon_{j} m_{j}, \varepsilon_{k} m_{k}\right)$ or $\left(\varepsilon_{i} m_{i}, \varepsilon_{k} m_{k}, \varepsilon_{j} m_{j}\right)$. Define $\operatorname{sign}(g, v)=\varepsilon_{i} \varepsilon_{j} \varepsilon_{k}$ for such a vertex. Define $\operatorname{sign}(\Gamma ; g)$ as the product of the signs associated with the vertices. Then

$$
Z_{n}\left(\left[M_{G} ; U^{(r)}\right]\right)=\left(\prod_{i=1}^{3 n} \frac{q_{i}}{p_{i}}\right) \sum_{\Gamma \in D_{e, n}, g \in G(\Gamma)} \frac{\operatorname{sign}(\Gamma ; g)}{2^{\theta(\Gamma)}}[\Gamma] .
$$

Now, Lemma 10.7 easily leads to the conclusion of the proof of Theorem 4.3.

- Orient $G$ arbitrarily. Under the hypotheses of Theorem 4.4, a contributing pairing for $\left(H_{1}, H_{2}\right) \in \mathcal{H}$ is a $2 / 3$-labelled Jacobi diagram $\Gamma$, equipped with a bijection from $V(\Gamma)$ to the set of components of $H_{1} \cup H_{1}^{-1} \cup H_{2}$ that maps a vertex with two adjacent edges labelled by $i$ and $j$ to a component of type $\left(\varepsilon_{i} m_{i}, \varepsilon_{j} m_{j}, f\right)$ or $\left(\varepsilon_{j} m_{j},-\varepsilon_{i} m_{i}, f\right)$. For a fixed 2/3-labelled Jacobi diagram $\Gamma$, there are $\sharp$ Aut $_{2 / 3}(\Gamma)$ bijections from $V(\Gamma)$ to the set of components of $H_{1} \cup H_{1}^{-1} \cup H_{2}$ that will correspond to the same pairing.

Let $\Gamma \in D_{2 / 3, n}$. Equip $\Gamma$ with an orientation. Let $G(\Gamma)$ be the set of maps $g$ from $V(\Gamma)$ to the set of components of $G$ that map a vertex $v$ whose adjacent edges are labelled by $(i, j$, nothing) (with respect to the orientation of $\Gamma$ ) to a component of 
type $\left(\varepsilon_{i} m_{i}, \varepsilon_{j} m_{j}, f\right)$ or $\left(\varepsilon_{j} m_{j},-\varepsilon_{i} m_{i}, f\right)$ of $G$. When $g \in G(\Gamma)$ is fixed, assign the framed oriented curve $\varepsilon_{i} \varepsilon_{j} f$ to the unlabelled edge of each $v \in V(\Gamma)$ as above. Then assign to each edge of $\Gamma$ the linking number of the two curves assigned to its half-edges (change a curve $f$ into its parallel $f_{\|}$, if the two curves coincide) and define $\operatorname{lk}(\Gamma ; g)$ as the product over the edges of $\Gamma$ of the associated linking numbers.

$$
Z_{n}\left(\left[M_{G} ; U^{(r)}\right]\right)=\left(\prod_{i=1}^{2 n} \frac{q_{i}}{p_{i}}\right)_{\Gamma \in D_{2 / 3, n}, g \in G(\Gamma)} \frac{1 \mathrm{k}(\Gamma ; g)}{\sharp \operatorname{Aut}_{2 / 3}(\Gamma)}[\Gamma] .
$$

Lemma 10.7 easily leads to the conclusion of the proof of Theorem 4.4 when the Seifert surfaces are associated with a presentation of the link by a graph that $\mu$-laces the unlink as in Proposition 10.8. Fortunately, this is enough to conclude the proof of Theorem 4.4 thanks to the following Proposition 11.1 that ensures that the right-hand side of the equality of Theorem 4.4 does not depend on the choice of the Seifert surfaces.

Let $n \in \mathbb{N}$. Let $D_{2 / 3, o, n}$ be the set of (isomorphism classes of) 2/3-labelled unoriented Jacobi diagrams whose labelled edges are oriented. Forgetting the edge orientations transforms an element $\Gamma$ of $D_{2 / 3, o, n}$ into an element $f(\Gamma)$ of $D_{2 / 3, n}$, and an element of $D_{2 / 3, n}$ comes from $2^{2 n} / \sharp A_{1} t_{2 / 3}(\Gamma)$ elements of $D_{2 / 3, o, n}$.

Let $L=\left(K_{i} ; p_{i} / q_{i}\right)_{i \in\{1,2,3, \ldots, 2 n\}}$ be a framed $2 n$-component algebraically split link in a rational homology sphere $M$. Assume that for any $\{i, j, k\} \subset\{1,2,3, \ldots, 2 n\}$, $\mu\left(K_{i}, K_{j}, K_{k}\right)=0$. Let $\left(F_{i}^{-}\right)_{i \in\{1,2,3, \ldots, 2 n\}} \cup\left(F_{i}^{+}\right)_{i \in\{1,2,3, \ldots, 2 n\}}$ be a collection of transverse surfaces such that, for any $i, F_{i}^{-}$and $F_{i}^{+}$are two Seifert surfaces of $K_{i}$ that do not meet the $K_{j}$ for $j \neq i$.

Let $\Gamma \in D_{2 / 3, o, n}$. Orient $\Gamma$. In such a $\Gamma$ the half-edges of the labelled edges inherit a label from the edge orientation. Namely, Edge $i$ goes from $i^{-}$to $i^{+}$.

For any vertex of $\Gamma$, whose half-edges are labelled by $\left(i^{\varepsilon}, j^{\eta}\right.$, nothing) with respect to the cyclic order induced by the orientation, assign the intersection curve $F_{i}^{\varepsilon} \cap F_{j}^{\eta}$ to its unlabelled half-edge. To any unlabelled edge that is now equipped with two intersection curves associate the linking number of these curves. Then define $\ell_{\Gamma}\left(\left(F_{i}^{-}, F_{i}^{+}\right)_{i=1, \ldots 2 n}\right)$ as the product over all the unlabelled edges of $\Gamma$ of the corresponding linking numbers. Note that $\ell_{\Gamma}\left(\left(F_{i}^{-}, F_{i}^{+}\right)_{i=1, \ldots 2 n}\right)[\Gamma]$ does not depend on the orientation of $\Gamma$.

When $F_{i}^{+}$is a parallel copy of $F_{i}^{-}$, then

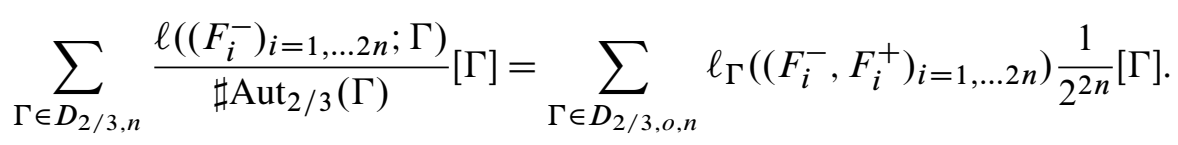


Proposition 11.1 With the notation and hypotheses above,

$$
\sum_{\Gamma \in D_{2 / 3, o, n}} \ell_{\Gamma}\left(\left(F_{i}^{-}, F_{i}^{+}\right)_{i=1, \ldots 2 n}\right) \frac{1}{2^{2 n}}[\Gamma]
$$

is independent of the choice of the surfaces $\left(F_{i}^{-}, F_{i}^{+}\right)_{i=1, \ldots 2 n}$ in the complement of $\bigcup_{j \neq i} K_{j}$, it only depends on $L$.

Proof We study the effect of changing a surface $F_{i}^{\varepsilon}$ to another Seifert surface $F^{\prime}$ of $K_{i}$ disjoint from the $K_{j}$ for $i \neq j$, and transverse to the other ones. Obviously, for any $\Gamma$, the only modified ingredient is the linking number associated with the unlabelled edge $e$ that shares a vertex with $i^{\varepsilon}$ that reads

$$
\pm \operatorname{lk}\left(F_{i}^{\varepsilon} \cap S_{1}, S_{2} \cap S_{3}\right)
$$

where $S_{1}, S_{2}$ and $S_{3}$ are the three other surfaces associated with the three other labelled half-edges containing the vertices of $e$.

Let us compute the variation of such a linking number. Recall $H_{2}\left(M \backslash \bigcup_{j=1,2, \ldots, 2 n} K_{j}\right)$ is generated by the homology classes of the boundaries $\partial N\left(K_{j}\right)$ of the tubular neighborhoods of the $K_{j}$, for $j \neq i$. Therefore the immersed oriented closed surface $\left(F_{i}^{\varepsilon} \cup-F^{\prime}\right)$ cobounds a 3-dimensional chain $C$ with some copies $\partial N\left(K_{j}\right)$. In particular, if $S_{1}$ is a Seifert surface for $K_{j(1)}$, the boundary of $C \cap S_{1}$ is the union of $\left(F^{\prime} \cap S_{1}-F_{i}^{\varepsilon} \cap S_{1}\right)$ and some copies of $K_{j(1)}$. Since all the Milnor triple linking numbers vanish, $\operatorname{lk}\left(K_{j(1)}, S_{2} \cap S_{3}\right)=0$, and

$$
\operatorname{lk}\left(F^{\prime} \cap S_{1}-F_{i}^{\varepsilon} \cap S_{1}, S_{2} \cap S_{3}\right)= \pm\left\langle C \cap S_{1}, S_{2} \cap S_{3}\right\rangle= \pm\left\langle C, S_{1} \cap S_{2} \cap S_{3}\right\rangle .
$$

Now, consider the two elements of $D_{2 / 3, o, n}$ obtained from $\Gamma$ by changing the neighborhood of $e$ in $\Gamma$ as in Figure 27.
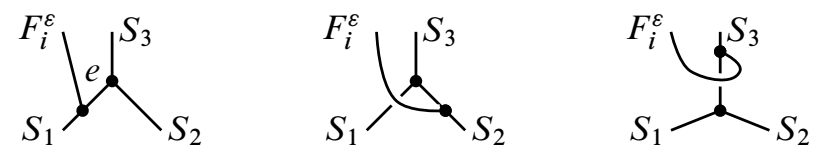

Figure 27

(Actually, since the current definition of Jacobi diagrams does not allow looped edges, some of the above graphs may not be Jacobi diagrams. In order to make this proof work, allow Jacobi diagrams with looped edges, and set them to be zero in $\mathcal{A}_{n}(\varnothing)$, so that the IHX relations involving such graphs are still valid and these graphs do not contribute to the sum of the statement.) 
Assume without loss, that the orientations of the three graphs coincide outside the neighborhood of $e$ and are induced by the figure at the shown vertices. Then the coefficients of these three elements of $D_{2 / 3, o, n}$ are perturbed in the same way. (Note that we did not need to take care about the above signs, they are well-defined in each step, and the result only depends on the cyclic order of $S_{1}, S_{2}, S_{3}$.) Since the sum of the corresponding oriented graphs vanishes in $\mathcal{A}_{n}(\varnothing)$ and since all the graphs of $D_{2 / 3, o, n}$ can be grouped in three-element sets as above, the sum of the statement is independent of the surfaces.

Similarly, we can show the following proposition.

Proposition 11.2 Let $L=\left(K_{0}, K_{1}, K_{2}, K_{3}\right)$ be a rationally algebraically split link whose three-component sublinks have Milnor triple linking number 0 in a rational homology sphere $M$. Let $a, b$ and $c$ be three real numbers such that $a+b+c=0$. Let $\Sigma_{i}$ be a Seifert surface for $K_{i}$ in the exterior of $L \backslash K_{i}$. Then

$$
\begin{aligned}
& v_{a b c}\left(K_{0}, K_{1}, K_{2}, K_{3}\right) \\
& \quad=a \operatorname{lk}\left(\Sigma_{0} \cap \Sigma_{1}, \Sigma_{2} \cap \Sigma_{3}\right)+b \operatorname{lk}\left(\Sigma_{0} \cap \Sigma_{2}, \Sigma_{3} \cap \Sigma_{1}\right)+c \operatorname{lk}\left(\Sigma_{0} \cap \Sigma_{3}, \Sigma_{1} \cap \Sigma_{2}\right)
\end{aligned}
$$

does not depend on the surfaces $\Sigma_{i}$ that satisfy the given assumption. The invariant $v_{a b c}$ satisfies the following properties.

- It is invariant under self-crossing changes of the components of $L$.

- If $M=S^{3}, v_{a b c}$ is the following combination of the Milnor invariants defined in [31],

$$
v_{a b c}=b \mu(10,23)-c \mu(01,23),
$$

where $\mu(01,23)=v_{1,0,-1}$ and $\mu(10,23)=v_{-1,1,0}$.

Proof The proof of Proposition 11.1 shows that $v_{a b c}$ does not depend on the surfaces and that it is therefore well-defined. Let us prove that $v_{a b c}$ does not vary under selfcrossing changes and is therefore a homotopy invariant of these four-component links. To study the effect of a self-crossing change on $K_{0}$ inside a ball $B$, choose the surfaces $\Sigma_{i}$ for $i>0$ so that they intersect $B$ as parallel tubes around one strand of $K_{0}$. Then their intersections like $\Sigma_{2} \cap \Sigma_{3}$ will not meet $B$, and will also bound a surface $\Sigma_{23}$ in the exterior of $K_{0}$ and $K_{1}$ that intersects $B$ as parallel tubes around the same strand of $K_{0}$. Now, $\Sigma_{1} \cap \Sigma_{23}$ does not meet $B$, and then

$$
\operatorname{lk}\left(\Sigma_{0} \cap \Sigma_{1}, \Sigma_{2} \cap \Sigma_{3}\right)= \pm \operatorname{lk}\left(K_{0}, \Sigma_{1} \cap \Sigma_{23}\right)
$$

does not vary under the considered crossing change of $K_{0}$. 
According to [31], if the ambient 3-manifold is $S^{3}$, there is a bijection from the set of homotopy classes of four-component algebraically split links $L$ whose threecomponent sublinks have Milnor triple linking number 0 to $\mathbb{Z} \oplus \mathbb{Z}$ that maps $L$ to $(\mu(01,23)(L), \mu(10,23)(L))$.

Furthermore, if $\left(K_{0}, K_{1}, K_{2}\right)$ is the trivial three-component link with meridians $\alpha_{0}$, $\alpha_{1}, \alpha_{2}$, and if the homotopy class of $K_{01}$ in the exterior of $\left(K_{0}, K_{1}, K_{2}\right)$ reads (with the notation of [31]),

$$
\alpha_{2}^{k_{0} k_{1}}=\alpha_{0} \alpha_{1} \alpha_{2}\left(\alpha_{0} \alpha_{1}\right)^{-1}\left(\alpha_{0} \alpha_{2}^{-1} \alpha_{0}^{-1}\right) \alpha_{2}\left(\alpha_{1} \alpha_{2}^{-1} \alpha_{1}^{-1}\right)=\left[\alpha_{0},\left[\alpha_{1}, \alpha_{2}\right]\right]
$$

then $\mu(01,23)\left(K_{0}, K_{1}, K_{2}, K_{01}\right)=1$ and $\mu(10,23)\left(K_{0}, K_{1}, K_{2}, K_{01}\right)=0$. More generally, if the homotopy class of $K_{3}$ reads $\left[\alpha_{0},\left[\alpha_{1}, \alpha_{2}\right]\right]^{\mu_{01}}\left[\alpha_{1},\left[\alpha_{0}, \alpha_{2}\right]\right]^{\mu_{10}}$, then $\mu(01,23)\left(K_{0}, K_{1}, K_{2}, K_{3}\right)=\mu_{01}$ and $\mu(10,23)\left(K_{0}, K_{1}, K_{2}, K_{3}\right)=\mu_{10}$. The link presented by the clasper of Figure 28 has the same properties as $\left(K_{0}, K_{1}, K_{2}, K_{01}\right)$

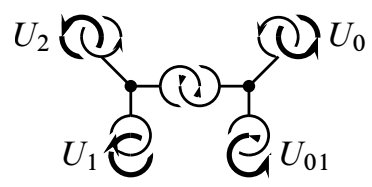

Figure 28

and, according to Lemma 10.7,

$$
v_{a b c}\left(\left(K_{0}, K_{1}, K_{2}, K_{01}\right)\right)=-c .
$$

More generally, if the homotopy class of $K_{3}$ reads $\left[\alpha_{0},\left[\alpha_{1}, \alpha_{2}\right]\right]^{\mu_{01}}\left[\alpha_{1},\left[\alpha_{0}, \alpha_{2}\right]\right]^{\mu_{10}}$, then

$$
v_{a b c}\left(K_{0}, K_{1}, K_{2}, K_{3}\right)=\left(b \mu_{10}-c \mu_{01}\right)\left(K_{0}, K_{1}, K_{2}, K_{3}\right) .
$$

\section{On the polynomial form of the knot surgery formula: proofs and remarks}

Proof of Theorem 5.1 Since the theorem easily follows from Theorem 4.1 for $n=1$, we assume $n \geq 2$. First assume $(p, q)=(1,0)$. A $1 / r$-surgery on $K$ is equivalent to $|r| \operatorname{sign}(r)$-surgeries on parallel copies on $K$. These parallel copies form an $|r|$-component boundary link $L$ bounding parallel copies of $F$. We have

$$
M\left(K ; \frac{1}{r}\right)=\sum_{J \subset L}(-1)^{\sharp J}[M ; J] .
$$


Up to elements of $\operatorname{Ker}\left(Z_{n}\right)$, we only consider the sublinks $J$ of $L$ with at most $n$ components, according to Theorem 4.1. There are

$$
\left(\begin{array}{c}
|r| \\
j
\end{array}\right)=\frac{|r|(|r|-1) \ldots(|r|-j+1)}{j !}
$$

sublinks $J$ of $L$ with $j$ components and they are all isomorphic to the boundary link $L_{j}$ whose components are framed by $\operatorname{sign}(r)$. This shows that

$$
Z_{n}\left(M\left(K ; \frac{1}{r}\right)\right)-Z_{n}(M)= \begin{cases}\sum_{i=1}^{n} Y_{n, 0}^{(i)}(K \subset M) r^{i} & \text { if } r \geq 0 \\ \sum_{i=1}^{n} Y_{n, 0}^{(i)}(K \subset M) r^{i} & \text { if } r \leq 0\end{cases}
$$

where

$$
Y_{n, 0}^{(n)-}=Y_{n, 0}^{(n)}=\frac{(-1)^{n}}{n !} Z_{n}\left(\left[M ; L_{n}\right]\right)
$$

is given by Theorem 4.1. Now, we prove that the two polynomial expressions, the one for $r>0$ and the one for $r<0$, coincide. Applying the above result to $M\left(K ; \frac{1}{r_{0}}\right)$ with $r_{0}<-n$, implies that for any $r \geq r_{0}$,

$$
Z_{n}\left(M\left(K ; \frac{1}{r}\right)\right)-Z_{n}\left(M\left(K ; \frac{1}{r_{0}}\right)\right)=\sum_{i=1}^{n} Y_{n, 0}^{(i)}\left(K \subset\left(M\left(K ; \frac{1}{r_{0}}\right)\right)\left(r-r_{0}\right)^{i}\right.
$$

The above result also implies that $Z_{n}\left(M\left(K ; \frac{1}{r}\right)\right)-Z_{n}\left(M\left(K ; \frac{1}{r_{0}}\right)\right)$ is

$$
\begin{array}{ll}
\sum_{i=1}^{n} Y_{n, 0}^{(i)}(K \subset M) r^{i}+Z_{n}(M)-Z_{n}\left(M\left(K ; \frac{1}{r_{0}}\right)\right) & \text { if } r \geq 0, \\
\sum_{i=1}^{n} Y_{n, 0}^{(i)-}(K \subset M) r^{i}+Z_{n}(M)-Z_{n}\left(M\left(K ; \frac{1}{r_{0}}\right)\right) & \text { if } r \leq 0 .
\end{array}
$$

Therefore the coefficients of the two polynomials coincide. This proves the existence of the polynomial expression with its given leading term for $(p, q)=(1,0)$. Applying this result in $M(K ; p / q)$ and using the fact that a $p /(q+r p)$-surgery on $K$ is equivalent to a $p / q$-surgery on $K$ and a $1 / r$-surgery on a parallel copy on $K$ gives a similar polynomial expression for $Z_{n}(M(K ; p /(q+r p)))-Z_{n}(M(K ; p / q))$ with the same leading coefficient since, according to Theorem 4.1 ,

$$
Z_{n}\left(\left[M ; L_{n}\right]\right)=Z_{n}\left(\left[M(K ; p / q) ; L_{n}\right]\right) .
$$


Now, up to polynomials in $r$ of degree less than $(n-1)$,

$$
\begin{aligned}
Z_{n}( & \left.M\left(K ; \frac{p}{q+r p}\right)\right)-Z_{n}(M) \\
= & \frac{(-1)^{n}}{n !} Z_{n}\left(\left[M ; L_{n}\right]\right) r^{n} \\
& \quad+\left(\frac{n(1-n)}{2} \frac{(-1)^{n}}{n !} Z_{n}\left(\left[M ; L_{n}\right]\right)+\frac{(-1)^{n-1}}{(n-1) !} Z_{n}\left(\left[M\left(K ; \frac{p}{q}\right) ; L_{n-1}\right]\right)\right) r^{n-1} \\
= & Y_{n, 0}^{(n)}(K \subset M)\left(r^{n}+\frac{n q}{p} r^{n-1}\right)+Y_{n, q / p}^{(n-1)}(K \subset M) r^{n-1} .
\end{aligned}
$$

Thus $Y_{n, q / p}^{(n-1)}(K \subset M)+n \frac{(-1)^{n} q}{n ! p} Z_{n}\left(\left[M ; L_{n}\right]\right)$

$$
=\frac{(-1)^{n-1}}{(n-1) !} Z_{n}\left(\left[M\left(K ; \frac{p}{q}\right) ; L_{n-1}\right]\right)+\frac{1}{2} \frac{(-1)^{n-1}(n-1)}{(n-1) !} Z_{n}\left(\left[M ; L_{n}\right]\right) \text {. }
$$

Then $Y_{n, q / p}^{(n-1)}(K \subset M)-Y_{n, 0}^{(n-1)}(K \subset M)$

$$
=\frac{(-1)^{n-1}}{(n-1) !}\left(\frac{q}{p} Z_{n}\left(\left[M ; L_{n}\right]\right)+Z_{n}\left(\left[M\left(K ; \frac{p}{q}\right) ; L_{n-1}\right]-\left[M ; L_{n-1}\right]\right)\right)
$$

where $\quad Z_{n}\left(\left[M\left(K ; \frac{p}{q}\right) ; L_{n-1}\right]-\left[M \sharp L(p,-q) ; L_{n-1}\right]\right)=-\frac{q}{p} Z_{n}\left(\left[M ; L_{n}\right]\right)$

by Theorem 4.1, and by additivity of $p^{c}\left(Z_{n}\right)=Z_{n}^{c}$ under connected sum, since $n \geq 2$,

$$
Z_{n}^{c}\left(\left[M \sharp L(p,-q) ; L_{n-1}\right]\right)=Z_{n}^{c}\left[M ; L_{n-1}\right] .
$$

Therefore $Y_{n, q / p}^{(n-1)^{c}}(K \subset M)=Y_{n, 0}^{(n-1)^{c}}(K \subset M)$.

The behaviour of $Y_{n, q / p}^{(i)}(K \subset M)$ under an orientation change of $M$ comes from the fact that $Z_{n}(-M)=(-1)^{n} Z_{n}(M)$, and the other assertions are easy to observe.

Remark 12.1 It is easy to see that $\left\langle\left\langle\bigsqcup_{i \in\{1, \ldots, n\}} I\left(F^{i}\right)\right\rangle\right\rangle$ is an invariant of the knot. First, it does not depend on the symplectic bases chosen for the Seifert surfaces because $H_{1}(F)$ may be identified to $H_{1}(F)^{*}$ via $(x \mapsto\langle x, \cdot\rangle)$, and therefore the tensor $\left(\sum_{i} x_{i} \otimes y_{i}-\sum_{i} y_{i} \otimes x_{i}\right)$ may be identified with the intersection form of the surface that lives in $H_{1}(F)^{*} \otimes H_{1}(F)^{*}$. Now, $\left\langle\bigsqcup_{i \in\{1, \ldots, n\}} I\left(F^{i}\right)\right\rangle$ is invariant under the addition of a hollow handle. (See Gordon [17, page 27] or Kearton and Lickorish [20] for a reference for the fact that for two Seifert surfaces of a knot $K$, there exists a third Seifert surface of $K$ that is obtained from the two former ones by adding hollow 
handles.) Indeed let $m$ be a meridian of a one-handle whose boundary is the union of the hollow handle and two disks, and let $\ell$ be a dual curve for it with respect to the intersection form of the stabilized surface $F$. Since the innermost copy of $m$ does not link any curve of the other copies of $F$, the pair $(m, \ell)$ does not contribute to the pairing. Now, the next innermost meridian does not link any other curve either... In such a way, it is easily seen that the pairs $(m, \ell)$ can be forgotten and this shows that $\left.\left\langle\bigsqcup_{i \in\{1, \ldots, n\}} I\left(F^{i}\right)\right\rangle\right\rangle$ is invariant under a stabilization of $F$ by addition of a hollow handle.

Proof of Proposition 5.3 Let $K_{0}=K_{\varnothing}$ be the positive desingularisation of $K^{s}$. Let $U^{(k)}$ be the trivial link that bounds a disjoint union of disks $D_{i}$ such that each $D_{i}$ meets $K^{s}$ exactly at one double point, and $\partial D_{i}$ does not algebraically link $K_{0}$, so that each desingularisation of $K^{s}$ is obtained from $K_{0}$ by surgery on a subset of $L=\left\{\left(U_{i} ;-1\right)\right\}_{i \in\{1, \ldots, k\}}$. Then

$$
\sum_{i=0}^{n} Y_{n, q / p}^{(i)}\left(K^{s} \subset M\right)\left(r+\frac{q}{p}\right)^{i}=Z_{n}\left(\left[M\left(K_{0} ; \frac{p}{q+r p}\right) ; L\right]\right) .
$$

Each $U_{i}$ bounds a genus one surface $\Sigma_{i}$ in $M \backslash K_{0}$ obtained from $D_{i}$ by tubing $K_{0}$, say in the $K_{i}^{\prime}$ part, where we fix the choice of the $K_{i}^{\prime}$ so that for any pair $\{i, j\}$, $K_{i}^{\prime} \cap K_{j}^{\prime}$ is connected.

Let us prove that such a choice is indeed possible for the $K_{i}^{\prime}$. Fixing the choice of $K_{i}^{\prime}$ amounts to choosing an interval of the circle between the two preimages of the double point $i$. If some of the two possible intervals for a double point $i$ does not contain a pair of preimages for another double point, pick such an interval. In the next steps, if some of the two intervals for a double point $i$ only contains pairs of preimages for another double point together with their associate already chosen intervals, then pick such an interval. It is easy to see that this process will stop when all the $K_{i}^{\prime}$ are chosen so that for any pair $\{i, j\}, K_{i}^{\prime} \cap K_{j}^{\prime}$ is connected.

Now, assume that the diameters of the tubes are all constant and different and that the tube for $U_{j}$ is thinner than the tube for $U_{i}$, if $K_{j}^{\prime}$ contains the two preimages of the double point $i$. Then $\Sigma_{i} \cap \Sigma_{j}$ is empty if the pair $\left(D_{i} \cap K_{0}\right)$ does not link the pair ( $D_{j} \cap K_{0}$ ), and it is a meridian curve of $K_{0}$ in $D_{j}$ otherwise. See Figure 29. Therefore, the $\mu$-invariants of the three-component sublinks of $L$ in $M\left(K_{0} ; p /(q+r p)\right)$ are zero and Theorem 4.4 can be used to compute $Z_{n}\left(\left[M\left(K_{0} ; p /(q+r p)\right) ; L\right]\right)$.

In particular, if $k>2 n, Z_{n}\left(\left[M\left(K_{0} ; p /(q+r p)\right) ; L\right]\right)=0$. Since the linking numbers between two intersection curves will be $\pm(q+r p) / p$ or zero, if $k=2 n$, $Z_{n}\left(\left[M\left(K_{0} ; p /(q+r p)\right) ; L\right]\right)$ is a monomial in $((q+r p) / p)^{n}$. 

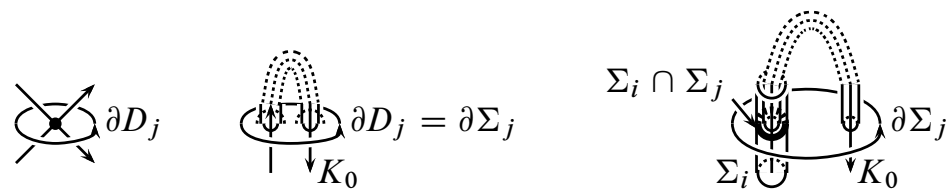

Figure 29

Proof of Proposition 5.4 In this case, the link $U^{(k)}$ of the previous proof is a boundary link in $M\left(K_{0} ; p /(q+r p)\right)$ because the produced genus one surfaces are disjoint. Then Theorem 4.1 can be applied. It implies the first part of the proposition. Use bases $\left(m_{i}, \ell_{i}\right)$ for the Seifert surfaces where $m_{i}$ is a 0 -framed meridian of $K_{0}$, and $\ell_{i}$ is a curve along the tube of $\Sigma_{i}$ and $D_{i}$ that is homotopic to $K_{i}^{s \prime}$, and that does not link $K_{0}$. In $M\left(K_{0} ; p /(q+r p)\right)$, the linking number of two meridians is $\pm(q+r p) / p$, the linking number of a meridian and a longitude is 0 or \pm 1 while the linking number of two longitudes is their linking number in $M$. Note that if one tube for $\Sigma_{j}$ goes inside another one for $\Sigma_{i}$ (if $K_{j}^{s, i} \neq K_{j}^{s \prime}$ ), and if $K_{j}^{\prime}$ is the positive desingularisation of $K_{j}^{s \prime}$ then $\operatorname{lk}\left(\ell_{i}, \ell_{j}\right)=\operatorname{lk}\left(\ell_{i}, K_{j}^{\prime}\right)=-\operatorname{lk}\left(\ell_{i}, K_{0}-K_{j}^{\prime}\right)=-\ell_{i j}\left(K^{s}\right)$. There are at most $n$ pairings of meridians. Furthermore, since there is at least one innermost meridian that cannot be paired with a longitude, there is at least one pairing of meridians. Now, the number of pairs of meridians coincides with the number of pairs of longitudes in a pairing.

As an example, we compute $Y_{2}^{(i) c}\left(K^{s}\right)$ where $K^{s}$ is a singular knot with two unlinked double points.

Proposition 12.2 Let $K^{s}$ be a singular knot with two unlinked double points.

$$
\begin{aligned}
\sum_{I \subset\{1,2\}}(-1)^{\sharp I} Z_{2}^{c}\left(M\left(K_{I} ; \frac{1}{r}\right)\right) & \\
= & \frac{1}{4}\left(\left(5 \ell_{12}\left(K^{s}\right)^{2}+2 \ell_{11}\left(K^{s}\right) \ell_{22}\left(K^{s}\right)\right) r^{2}-\ell_{12}\left(K^{s}\right) r\right) 80 .
\end{aligned}
$$

Proof Use the strategy and the notation of the proofs of the two previous propositions. Choose Seifert surfaces of the two knots of the crossing changes with disjoint tubes whose longitudes $\ell_{1}$ and $\ell_{2}$ are homotopic to $K_{1}^{s, 2}$ and $K_{2}^{s, 1}$, respectively, so that

$$
\begin{aligned}
\operatorname{lk}\left(\ell_{1}, \ell_{2}\right) & =\operatorname{lk}\left(\ell_{1}, \ell_{2}^{+}\right)=\operatorname{lk}\left(\ell_{1}^{+}, \ell_{2}\right)=\ell_{12}\left(K^{s}\right), \\
\operatorname{lk}\left(m_{1}, m_{2}\right) & =-r=\operatorname{lk}\left(m_{i}, m_{i}^{+}\right), \\
\operatorname{lk}\left(\ell_{i}, \ell_{i}^{+}\right) & =-\ell_{i i}\left(K^{s}\right),
\end{aligned}
$$




$$
\begin{aligned}
& \operatorname{lk}\left(m_{i}, \ell_{i}^{+}\right)=\operatorname{lk}\left(m_{1}, \ell_{2}\right)=\operatorname{lk}\left(m_{2}, \ell_{1}\right)=0, \\
& \operatorname{lk}\left(m_{i}^{+}, \ell_{i}\right)=1 .
\end{aligned}
$$

Then, according to Theorem 4.1,

$$
\sum_{I \subset\{1,2\}}(-1)^{\sharp I} Z_{2}^{c}\left(M\left(K_{I} ; \frac{1}{r}\right)\right)=\frac{1}{4} p^{c}\left(\left\langle\left\langle\begin{array}{cc}
m_{1}^{+} & \ell_{1}^{+} \\
\ell_{1}^{\ell_{2}^{+}} & < \\
m_{1} & <\ell_{2}^{+} \\
\ell_{2} \\
m_{2}
\end{array} \|\right) .\right.\right.
$$

Note that $m_{1}$ and $m_{2}$ must be paired to another meridian. Then the right-hand side of the equality can be rewritten as

$$
\frac{r^{2}}{4} p^{c}\left(\left\|\left(\begin{array}{cc}
\ell_{1}^{+} & \ell_{2}^{+} \\
\ell_{1} & \ell_{2}
\end{array}\right\rceil+\left[\begin{array}{cc}
\ell_{1}^{+} & \ell_{2}^{+} \\
\ell_{1} & \ell_{2}
\end{array}\right]+\left[\begin{array}{cc}
\ell_{1}^{+} & \ell_{2} \\
\ell_{1} & \ell_{2}^{+}
\end{array}\right]\right\|\right)-\frac{r}{4} \ell_{12}\left(K^{s}\right) Q 0 .
$$

Indeed, either two pairs of meridians are paired together-leading to the quadratic contribution in $r$ above - or there is one pair of meridians, it is necessarily $\left(m_{1}, m_{2}\right)$ and in this case $m_{1}^{+}$must be paired with $\ell_{1}$ and $m_{2}^{+}$must be paired with $\ell_{2}$. Then $\ell_{1}^{+}$and $\ell_{2}^{+}$must be paired together, and this yields the linear contribution above. $\square$

\section{Computation of the Casson-Walker knot invariant}

Let $K$ be an order $O_{K}$ knot in a rational homology sphere $M$. Let $\widetilde{M \backslash K}$ be the infinite cyclic covering of $M \backslash K$. Denote the action of the homotopy class of the meridian of $K$ on $H_{1}(M \backslash K ; \mathbb{Q})$ as the multiplication by $t$ so that a generator of $H_{1}(M \backslash K) /$ Torsion acts as the multiplication by $t^{1 / O_{K}}$. As in [27, Chapter 2], define the Alexander polynomial $\Delta(K)$ of $K$ as the order of the $\mathbb{Q}\left[t^{ \pm 1 / O_{K}}\right]$-module $H_{1}(\widetilde{M \backslash K} ; \mathbb{Q})$ normalized so that

$\Delta(K)(1)=\left|\operatorname{Torsion}\left(H_{1}(M \backslash K)\right)\right|=\frac{\left|H_{1}(M)\right|}{O_{K}}$ and $\Delta(K)\left(t^{1 / O_{K}}\right)=\Delta(K)\left(t^{-1 / O_{K}}\right)$.

Then the formula of [27, pages 12-13] implies the following lemma.

Lemma 13.1 For any knot $K$ such that $\operatorname{lk}(K, K) \in \mathbb{Z}$ in a rational homology sphere $M$, for any pair $(p, q)$ of coprime integers such that $q \neq 0$.

$\lambda(M(K ; p / q))-\lambda(M)=\frac{q}{p}\left(\frac{O_{K}}{\left|H_{1}(M)\right|} \frac{\Delta^{\prime \prime}(K)(1)}{2}-\frac{1}{24 O_{K}^{2}}+\frac{1}{24}\right)+\lambda(L(p,-q))$. 
Proof Recall that $\lambda(M)=\bar{\lambda}(M) /\left|H_{1}(M)\right|$ where $\left|H_{1}(M)\right|$ is the cardinality of $H_{1}(M ; \mathbb{Z})$ and $\bar{\lambda}$ is the extension of $\left|H_{1}(M)\right| \lambda$ to oriented closed 3-manifolds that is denoted by $\lambda$ in [27]. For any knot $K$ in a rational homology sphere $M$, according to $[27,1.4 .8, \mathrm{~T} 2]$, for $q>0$,

$$
\begin{aligned}
& \lambda(M(K ; p / q))-\lambda(M) \\
& =\frac{q}{p}\left(\frac{O_{K}}{\left|H_{1}(M)\right|} \frac{\Delta^{\prime \prime}(K)(1)}{2}-\frac{1}{24 O_{K}^{2}}-\frac{p^{2}+1}{24 q^{2}}\right)+\frac{\operatorname{sign}(p q)}{8}+\frac{s(p-q l k(K, K), q)}{2}
\end{aligned}
$$

where the Dedekind sum $s(p-q l k(K, K), q)$ is defined in [34] by Rademacher and Grosswald (and in $[27,1.4 .5])$. This formula makes clear that

$$
\lambda(M(K ; p / q))-\lambda(M)=\frac{q}{p}\left(\frac{O_{K}}{\left|H_{1}(M)\right|} \frac{\Delta^{\prime \prime}(K)(1)}{2}-\frac{1}{24 O_{K}^{2}}+\frac{1}{24}\right)+f(p, q)
$$

for some $f(p, q)$ that depends neither on the knot $K$ with self-linking number 0 nor on its ambient manifold $M$. Applying this formula to the trivial knot $U$ of $S^{3}$ concludes the proof of the lemma.

We now express $\Delta(K)$ from the Seifert form of a Seifert surface for $K$ in the following proposition.

Proposition 13.2 Let $K$ be a knot of order $d$, with self-linking number $(-a / b) \in$ $\mathbb{Q} / \mathbb{Z}$, where $a$ and $b$ are coprime integers, in a rational homology sphere $M$. Let $N(K)$ be a tubular neighborhood of $K$. Let $\Sigma$ be a surface in $M$ whose boundary is made of $(d / b)$ parallel copies of a primitive curve of $\partial N(K)$. Let $\mathcal{B}_{s}$ be a symplectic basis for $H_{1}(\Sigma) / H_{1}(\partial \Sigma)$, and let

$$
\Delta_{\Sigma}(\tau)=\operatorname{det}\left[\operatorname{lk}\left(\tau^{1 / 2} b^{\prime+}-\tau^{-1 / 2} b^{\prime-}, b\right)\right]_{\left(b, b^{\prime}\right) \in \mathcal{B}_{s}^{2}}
$$

where $b^{\prime+}$ (resp. $b^{--}$) is a representative of $b^{\prime}$ pushed away from $\Sigma$ in the direction of the positive (resp. negative) normal direction to $\Sigma$. Then

$$
\frac{d}{\left|H_{1}(M)\right|} \Delta(K)=\Delta_{\Sigma}\left(t^{1 / d}\right) \frac{b}{d} \frac{t^{1 /(2 b)}-t^{-1 /(2 b)}}{t^{1 /(2 d)}-t^{-1 /(2 d)}} .
$$

Proof First assume that the self-linking number of $K$ is zero. Let $N(K)$ be a tubular neighborhood of $K$. There exists a genus $g$ surface $\Sigma$ in $M$ whose boundary is made of $d$ parallel copies of $K$. Consider a collar $\Sigma \times[-1,1]$ in $M$ such that

$$
(\Sigma \times[-1,1]) \cap N(K)=\partial \Sigma \times[-1,1] .
$$

Let $Y=M \backslash(N(K) \cup \Sigma \times]-1,1[)$. 
The infinite cyclic covering $\tilde{X}$ of $M \backslash N(K)$ can be seen as

$$
\left(\coprod_{k \in \mathbb{Z}} h^{k}(Y) \coprod \coprod_{k \in \mathbb{Z}} h^{k}(\Sigma \times[-1,1])\right) / \cong
$$

where $h$ is a generator of the group of automorphisms of the covering $\tilde{X}$ and $\cong$ yields the following identifications:

$$
\begin{aligned}
h^{k}((\sigma \in \Sigma, 1) \in Y) & \cong h^{k}((\sigma \in \Sigma, 1) \in(\Sigma \times[-1,1])), \\
h^{k}((\sigma \in \Sigma,-1) \in Y) & \cong h^{k+1}((\sigma \in \Sigma,-1) \in(\Sigma \times[-1,1])) .
\end{aligned}
$$

Then it is easy to see that, if the action of $h$ on $H_{1}(\tilde{X} ; \mathbb{Q})$ is denoted as a multiplication by $\tau$,

$$
H_{1}(\tilde{X} ; \mathbb{Q})=\frac{H_{1}(Y ; \mathbb{Q}) \otimes \mathbb{Q}\left[\tau, \tau^{-1}\right]}{\left(\bigoplus_{b \in \mathcal{B}}\left(\tau b^{+}-b^{-}\right) \mathbb{Q}\right) \otimes \mathbb{Q}\left[\tau, \tau^{-1}\right]},
$$

as a $\mathbb{Q}\left[\tau, \tau^{-1}\right]$-module, where $\mathcal{B}$ is a basis of $H_{1}(\Sigma)$ and, for $b \in \mathcal{B}, b^{+}$(resp. $b^{-}$) denotes the class of $b$ in $H_{1}(\Sigma \times\{1\})$ (resp. in $H_{1}(\Sigma \times\{-1\})$ ).

In particular, if $\mathcal{C}$ is a basis of $H^{1}(Y ; \mathbb{Q})$, then

$$
\Delta(K)\left(\tau=t^{1 / d}\right)=\operatorname{det}\left[\tau^{1 / 2} c\left(b^{+}\right)-\tau^{-1 / 2} c\left(b^{-}\right)\right]_{(c, b) \in \mathcal{C} \times \mathcal{B}}
$$

up to a multiplication by a unit of $\mathbb{Q}\left[\tau, \tau^{-1}\right]$.

\section{Computation of $\boldsymbol{H}^{\mathbf{1}}(\boldsymbol{Y} ; \mathbb{Q}) \quad$ Let $Z=M \backslash(\Sigma \times]-1,1[)$.}

The collar $\Sigma \times[-1,1]$ is a genus $(2 g+d-1)$-handlebody whose $H_{1}$ has a basis $\mathcal{B}$ made of the classes $\ell_{1}, \ell_{2}, \ldots, \ell_{d-1}$ of $(d-1)$ boundary components of $\Sigma$, and a symplectic basis $\mathcal{B}_{s}$ for $H_{1}(\Sigma) / H_{1}(\partial \Sigma)$. Therefore, $Z$ has the rational homology of a genus $(2 g+d-1)$-handlebody and $H^{1}(Z ; \mathbb{Q})$ is freely generated by the linking numbers with the elements of $\mathcal{B}$.

Use the following exact sequence to compute $H^{1}(Y ; \mathbb{Q})$ :

$$
H^{1}(Z, Y ; \mathbb{Q}) \hookrightarrow H^{1}(Z ; \mathbb{Q}) \rightarrow H^{1}(Y ; \mathbb{Q}) \rightarrow H^{2}(Z, Y ; \mathbb{Q}) \rightarrow 0 .
$$

The pair $(Z, Y)$ has the homology of the pair $(N(K), \partial N(K) \backslash(\partial \Sigma \times[-1,1]))$ where $\partial N(K) \backslash(\partial \Sigma \times[-1,1])$ is a disjoint union of $d$ annuli $A\left(\ell_{i}^{++}\right)$whose cores are parallels $\ell_{1}^{++}, \ell_{2}^{++}, \ldots, \ell_{d}^{++}$of $K$, and such that

$$
\partial A\left(\ell_{i}^{++}\right)=\ell_{i}^{+}-\ell_{i+1}^{-}
$$


(where $\left.\ell_{d+1}^{-}=\ell_{1}^{-}\right)$. In particular,

$$
H_{j}(Z, Y)= \begin{cases}0 & \text { if } j \neq 1,2, \\ \bigoplus_{i=2}^{d} \mathbb{Z} c_{i} & \text { if } j=1, \\ \bigoplus_{i=2}^{d} \mathbb{Z} B_{i} & \text { if } j=2,\end{cases}
$$

where $c_{i}$ is the class of a path from $\ell_{1}^{++}$to $\ell_{i}^{++}$in $N(K)$, and $B_{i}$ is the class of an annulus whose boundary is $\left(\ell_{i}^{++}-\ell_{1}^{++}\right)$.

The image of $H^{1}(Z, Y ; \mathbb{Q}) \hookrightarrow H^{1}(Z ; \mathbb{Q})$ is freely generated by the algebraic intersections $\left\langle\cdot,-A\left(\ell_{i}^{++}\right)\right\rangle=\operatorname{lk}\left(\cdot, \ell_{i+1}^{-}-\ell_{i}^{+}\right)$for $i \in 2, \ldots, d$.

For $i \geq 2$, consider a curve $e_{i}$ that goes from $\ell_{i}$ to $\ell_{i+1}$ in $\Sigma$ and that avoids the chosen geometric symplectic basis of $H_{1}(\Sigma) / H_{1}(\partial \Sigma)$, and consider a closed loop $\mu_{i}$ in $N(K) \cup \Sigma \times[-1,1]$ that equals $e_{i}$ outside $N(K)$. See Figure 30 .

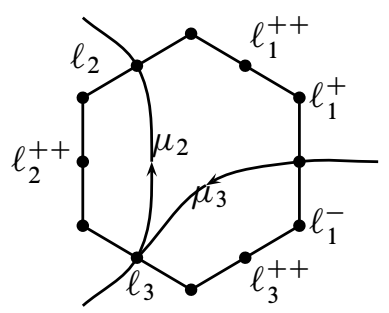

Figure 30

Then $\operatorname{lk}\left(\partial B_{i}=\ell_{i}^{++}-\ell_{1}^{++}, \mu_{j}\right)=\delta_{i j}$. Therefore the map $H^{1}(Y ; \mathbb{Q}) \rightarrow H^{2}(Z, Y ; \mathbb{Q})$ admits a section whose image is $\bigoplus_{i=2}^{d} \mathbb{Q} \operatorname{lk}\left(\cdot, \mu_{i}\right)$.

Thus

$$
H^{1}(Y ; \mathbb{Q})=\bigoplus_{b \in \mathcal{B}_{s}} \mathbb{Q} \operatorname{lk}(\cdot, b) \oplus \bigoplus_{i=2}^{d} \mathbb{Q} \operatorname{lk}\left(\cdot, \mu_{i}\right) .
$$

Since $\operatorname{lk}\left(\ell_{i}^{ \pm}, b\right)=0$ for any $b \in \mathcal{B}_{s}$, up to units of $\mathbb{Q}\left[t^{ \pm 1 / O_{K}}\right]$,

$$
\Delta(K)=\Delta_{\Sigma}(\tau) \Delta(d)
$$

with

$$
\Delta(d)=\operatorname{det}\left[\operatorname{lk}\left(\tau^{1 / 2} \ell_{i}^{+}-\tau^{-1 / 2} \ell_{i}^{-}, \mu_{j}\right)\right]_{(i, j) \in\{2, \ldots, d\}^{2}}
$$

where $\ell_{i}^{+}=\ell_{i}^{++}$and $\ell_{i}^{-}=\ell_{i-1}^{++}$.

\section{Sublemma 1}

$$
\Delta(d)=\frac{\tau^{d / 2}-\tau^{-d / 2}}{d\left(\tau^{1 / 2}-\tau^{-1 / 2}\right)} .
$$


Proof of the sublemma By pushing $\mu_{j}$ along the negative normal of the Seifert surface of $\ell_{1}^{++}$(or $K$ ) we see that $\operatorname{lk}\left(\ell_{1}^{++}, \mu_{j}\right)=-1 / d$.

Set $z=\tau^{1 / 2}-\tau^{-1 / 2}$ and $\rho=\tau^{1 / 2}, \Delta(d)$ is the determinant of the following matrix $\left[\Delta_{i j}\right]_{(i, j) \in\{2, \ldots, d\}^{2}}$ where

$$
\Delta_{2 j}=\operatorname{lk}\left(\rho \ell_{2}^{++}-\rho^{-1} \ell_{1}^{++}, \mu_{j}\right)=\rho \delta_{2 j}-\frac{z}{d},
$$

and for $i>2$,

$$
\Delta_{i j}=\operatorname{lk}\left(\rho\left(\ell_{i}^{++}-\ell_{2}^{++}\right)-\rho^{-1}\left(\ell_{i-1}^{++}-\ell_{1}^{++}\right), \mu_{j}\right)=\rho\left(\delta_{i j}-\delta_{2 j}\right)-\rho^{-1} \delta_{(i-1) j},
$$

that is for $d=5$,

$$
\left[\Delta_{i j}\right]=\left[\begin{array}{cccc}
\rho-\frac{z}{d} & -\frac{z}{d} & -\frac{z}{d} & -\frac{z}{d} \\
-\rho-\rho^{-1} & \rho & 0 & 0 \\
-\rho & -\rho^{-1} & \rho & 0 \\
-\rho & 0 & -\rho^{-1} & \rho
\end{array}\right]
$$

$\Delta(2)=\left(\rho+\rho^{-1}\right) / 2$ and $\Delta(3)=\left(\tau+\tau^{-1}+1\right) / 3$.

In general the expansion with respect to the first column gives that

$$
\begin{aligned}
\Delta(d)=\left(\rho-\frac{z}{d}\right) \rho^{(d-2)} & -\frac{z}{d}\left(\rho+\rho^{-1}\right)\left(\sum_{j=3}^{d} \rho^{(d-j)-(j-3)}\right) \\
& -\frac{z}{d} \rho \sum_{i=4}^{d} \rho^{i-3} \sum_{j=i}^{d} \rho^{(d-j)-(j-i)}
\end{aligned}
$$

where

$$
\rho^{-1}\left(\sum_{j=3}^{d} \rho^{(d-j)-(j-3)}\right)=\sum_{j=3}^{d} \rho^{(d-2 j+2)}=\rho^{(2-d)}+\rho^{(4-d)}+\cdots+\rho^{(d-4)} .
$$

Thus,

$$
\begin{gathered}
\Delta(d)=\rho^{(d-1)}-\frac{z}{d} \rho^{(d-2)}-\frac{\rho^{(d-3)}-\rho^{(1-d)}}{d}-\frac{z}{d} \rho^{-2} \sum_{i=3}^{d} \sum_{j=i}^{d} \rho^{(d+2 i-2 j)} . \\
\sum_{i=3}^{d} \sum_{j=i}^{d} \rho^{(d+2 i-2 j)}=(d-2) \rho^{d}+(d-3) \rho^{d-2} \\
+(d-4) \rho^{d-4}+\cdots+\rho^{(6-d)}
\end{gathered}
$$




$$
\begin{gathered}
z \sum_{i=3}^{d} \sum_{j=i}^{d} \rho^{(d+2 i-2 j)}=(d-2) \rho^{(d+1)}-\rho^{(d-1)}-\rho^{(d-3)}-\cdots-\rho^{(5-d)} \\
\begin{aligned}
& d \Delta(d)=d \rho^{(d-1)}-\rho^{(d-1)}+\rho^{(1-d)}-(d-2) \rho^{(d-1)} \\
&+\rho^{(d-3)}+\rho^{(d-5)}+\cdots+\rho^{(3-d)}
\end{aligned} \\
=\rho^{(d-1)}+\rho^{(d-3)}+\rho^{(d-5)}+\cdots+\rho^{(3-d)}+\rho^{(1-d)}=\frac{\tau^{d / 2}-\tau^{-d / 2}}{\left(\tau^{1 / 2}-\tau^{-1 / 2}\right)}
\end{gathered}
$$

Going back to the proof of Proposition 13.2, since $\Delta(K)\left(t=\tau^{d}\right)(1)=\left|H_{1}(M)\right| / d$,

$$
\Delta(K)(t)=\frac{\left|H_{1}(M)\right|}{d} \frac{t^{1 / 2}-t^{-1 / 2}}{d\left(t^{1 /(2 d)}-t^{-1 /(2 d)}\right)} \Delta_{\Sigma}(\tau) .
$$

and Proposition 13.2 is proved in the self-linking number 0 case. Let us now deduce the general case from this case. Let $K$ be a knot with order $d$ and with self-linking number $(-a / b)$ where $a$ and $b$ are coprime. Let $m$ be a meridian of $K$, there exist a parallel $L$ of $K$ and a surface $\Sigma$ in $M \backslash K$ whose boundary is made of $(d / b)$ parallel copies of $a m+b L$. Then there exists a primitive curve $m_{J}$ such that $\left\langle m_{J}, a m+b L\right\rangle=1$. Let $J$ be the knot with meridian $m_{J}$ and with complement $M \backslash K$. This knot has order $(d / b)$ and self-linking number 0 . Its Alexander polynomial is then given by the proposition. Furthermore, since it satisfies $\Delta(J)(1)=\left|\operatorname{Torsion}\left(H_{1}(M \backslash K)\right)\right|=$ $\Delta(K)(1), \Delta(J)\left(t_{J}=\tau^{d / b}\right)=\Delta(K)\left(t_{K}=\tau^{d}\right)$. Then $\Delta(K)\left(t_{K}\right)=\Delta(J)\left(t_{J}=t_{K}^{1 / b}\right)$ and we are done.

Proposition 13.2 implies the following lemma that together with Lemma 13.1 proves Proposition 6.1 for $n=1$. We use the notation of Section 6 .

Lemma 13.3 Under the assumptions of Proposition 13.2,

$$
\frac{d}{\left|H_{1}(M)\right|} \frac{\Delta^{\prime \prime}(K)(1)}{2}=\frac{\langle\langle I(\Sigma)\rangle\rangle_{1}}{2 d^{2}}+\frac{1}{24 b^{2}}-\frac{1}{24 d^{2}} .
$$

Proof First note that when $K$ is null-homologous, $O_{K}=d=b=1$. Then since $\lambda=W_{1} \circ Z_{1}$, Lemma 13.1 and Theorem 4.1 together imply that

$$
\frac{1}{\left|H_{1}(M)\right|} \frac{\Delta^{\prime \prime}(K)(1)}{2}=\frac{\langle\langle I(\Sigma)\rangle\rangle_{W_{1}}}{2} \text {. }
$$

Therefore, according to Proposition 13.2 (that is well-known in this case),

$$
\frac{\Delta_{\Sigma}^{\prime \prime}(1)}{2}=\frac{\left\langle\langle I(\Sigma)\rangle_{W_{1}}\right.}{2}
$$


Then since $\Delta_{\Sigma}(t)=\Delta_{\Sigma}\left(t^{-1}\right)$,

$$
\Delta_{\Sigma}(\exp (u))=1+\frac{\langle\langle I(\Sigma)\rangle\rangle_{W_{1}}}{2} u^{2}+O(4)
$$

where $O(4)$ stands for an element of $u^{4} \mathbb{Q}[[u]]$, and this formula remains true for any $\Sigma$ as in the statement of Proposition 13.2. Since

$$
\exp (u)^{1 /(2 d)}-\exp (u)^{-1 /(2 d)}=\frac{u}{d}\left(1+\frac{u^{2}}{24 d^{2}}+O(4)\right),
$$

it is easy to conclude.

Now that Proposition 6.1 is shown for $n=1$, let us prove it for $n=2$. By the formula that is recalled in the beginning of the proof of Lemma 13.1,

$$
\lambda(M(K ; p / q))-\lambda(M)=\frac{q}{p} \frac{O_{K}}{\left|H_{1}(M)\right|} \frac{\Delta^{\prime \prime}(K)(1)}{2}+f\left(p, q, \operatorname{lk}(K, K), O_{K}\right)
$$

for some $f\left(p, q, \operatorname{lk}(K, K), O_{K}\right)$ that only depends on $p, q, \operatorname{lk}(K, K), O_{K}$, and that therefore does not change under surgery on a knot $K_{2}$ that does not link $K$ algebraically, so that

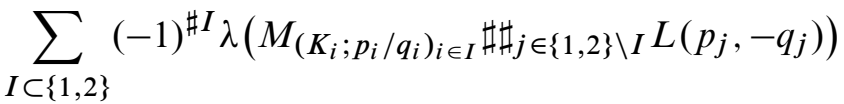

$$
\begin{aligned}
& =\sum_{I \subset\{1,2\}}(-1)^{\sharp I} \lambda\left(M_{\left.\left(K_{i} ; p_{i} / q_{i}\right)_{i \in I}\right)}\right) \\
& =\frac{q_{1}}{p_{1}}\left(\frac{O_{K_{1}} \Delta^{\prime \prime}\left(K_{1} \subset M\left(K_{2} ; p_{2} / q_{2}\right)\right)(1)}{2\left|H_{1}\left(M\left(K_{2} ; p_{2} / q_{2}\right)\right)\right|}-\frac{O_{K_{1}} \Delta^{\prime \prime}\left(K_{1} \subset M\right)(1)}{2\left|H_{1}(M)\right|}\right) \\
& =\frac{q_{1}}{2 p_{1} O_{K_{1}}^{2}}\left(\left\langle\left\langle I\left(\Sigma_{1}\right) \subset M\left(K_{2} ; p_{2} / q_{2}\right)\right\rangle\right\rangle_{W_{1}}-\left\langle\left\langle I\left(\Sigma_{1}\right) \subset M\right\rangle\right\rangle_{W_{1}}\right)
\end{aligned}
$$

according to Lemma 13.3. Therefore, Proposition 6.1 for $n=2$ follows from the following lemma.

Lemma 13.4 Under the assumptions of Proposition 6.1, $\left\langle\left\langle I\left(\Sigma_{1}\right) \subset M\left(K_{2} ; p / q\right)\right\rangle\right\rangle_{W_{1}}-\left\langle\left\langle I\left(\Sigma_{1}\right) \subset M\right\rangle\right\rangle_{W_{1}}=-\frac{2 q}{d_{2}^{2} p} \operatorname{lk}\left(\Sigma_{1} \cap \Sigma_{2},\left(\Sigma_{1} \cap \Sigma_{2}\right)_{\|}\right)$.

Proof Let $\left(x_{i}, y_{i}\right)_{i \in\{1, \ldots, g\}}$ be a symplectic basis for $H_{1}\left(\Sigma_{1}\right) / H_{1}\left(\partial \Sigma_{1}\right)$. Because of the variation of linking numbers after surgery recalled in Lemma 9.5, the variation of 
the expression of $\left\langle\left\langle I\left(\Sigma_{1}\right)\right\rangle\right\rangle_{W_{1}}$ given before Proposition 6.1 reads

$$
\begin{aligned}
\left\langle\left\langle I\left(\Sigma_{1}\right) \subset M\left(K_{2} ; p / q\right)\right\rangle\right\rangle_{W_{1}}-\left\langle\left\langle I\left(\Sigma_{1}\right) \subset M\right\rangle\right\rangle_{W_{1}} \\
=2 \frac{q^{2}}{p^{2}} \sum_{(j, k) \in\{1,2, \ldots, g\}^{2}} \operatorname{lk}\left(x_{j}, K_{2}\right) \operatorname{lk}\left(K_{2}, x_{k}^{+}\right) \operatorname{lk}\left(y_{j}, K_{2}\right) \operatorname{lk}\left(K_{2}, y_{k}^{+}\right) \\
\quad-2 \frac{q^{2}}{p^{2}} \sum_{(j, k) \in\{1,2, \ldots, g\}^{2}} \operatorname{lk}\left(x_{j}, K_{2}\right) \operatorname{lk}\left(K_{2}, y_{k}^{+}\right) \operatorname{lk}\left(y_{j}, K_{2}\right) \operatorname{lk}\left(K_{2}, x_{k}^{+}\right) \\
\quad-2 \frac{q}{p} \sum_{(j, k) \in\{1,2, \ldots, g\}^{2}}\left(\operatorname{lk}\left(x_{j}, K_{2}\right) \operatorname{lk}\left(K_{2}, x_{k}^{+}\right) \operatorname{lk}\left(y_{j}, y_{k}^{+}\right)\right. \\
-2 \frac{q}{p} \sum_{(j, k) \in\{1,2, \ldots, g\}^{2}}\left(\operatorname{lk}\left(x_{j}, x_{k}^{+}\right) \operatorname{lk}\left(y_{j}, K_{2}\right) \operatorname{lk}\left(K_{2}, y_{k}^{+}\right) \operatorname{lk}\left(K_{2}, y_{k}^{+}\right) \operatorname{lk}\left(y_{j}, x_{k}^{+}\right)\right) \\
\left.-\operatorname{lk}\left(x_{j}, y_{k}^{+}\right) \operatorname{lk}\left(y_{j}, K_{2}\right) \operatorname{lk}\left(K_{2}, x_{k}^{+}\right)\right)
\end{aligned}
$$

where the quadratic part in $q / p$ is obviously zero. On the other hand, when $c \in H_{1}\left(\Sigma_{1}\right)$,

$$
\left\langle c, \Sigma_{1} \cap \Sigma_{2}\right\rangle_{\Sigma_{1}}=d_{2} \operatorname{lk}\left(c, K_{2}\right) .
$$

Therefore in $H_{1}\left(\Sigma_{1}\right)$,

$$
\Sigma_{1} \cap \Sigma_{2}=d_{2} \sum_{i=1}^{g}\left(\operatorname{lk}\left(x_{i}, K_{2}\right) y_{i}-\operatorname{lk}\left(y_{i}, K_{2}\right) x_{i}\right)
$$

and

$$
\begin{aligned}
\operatorname{lk}\left(\Sigma_{1} \cap \Sigma_{2},\left(\Sigma_{1} \cap \Sigma_{2}\right)^{+}\right) \\
=d_{2}^{2} \sum_{(j, k) \in\{1,2, \ldots, g\}^{2}} \operatorname{lk}\left(\operatorname{lk}\left(x_{j}, K_{2}\right) y_{j}-\operatorname{lk}\left(y_{j}, K_{2}\right) x_{j}\right. \\
\left.\operatorname{lk}\left(x_{k}, K_{2}\right) y_{k}^{+}-\operatorname{lk}\left(y_{k}, K_{2}\right) x_{k}^{+}\right) .
\end{aligned}
$$

Then Proposition 6.1 is proved for $n=2$. Since

$$
\begin{aligned}
\operatorname{lk}_{M\left(K_{3} ; p_{3} / q_{3}\right)}\left(\Sigma_{1} \cap \Sigma_{2},\left(\Sigma_{1} \cap \Sigma_{2}\right)_{\|}\right)-\operatorname{lk}_{M}\left(\Sigma_{1} \cap \Sigma_{2},\right. & \left.\left(\Sigma_{1} \cap \Sigma_{2}\right)_{\|}\right) \\
& =-\frac{q_{3}}{p_{3}} \operatorname{lk}_{M}\left(\Sigma_{1} \cap \Sigma_{2}, K_{3}\right)^{2} \\
& =-\frac{q_{3}}{d_{3}^{2} p_{3}}\left\langle\Sigma_{1}, \Sigma_{2}, \Sigma_{3}\right\rangle^{2}
\end{aligned}
$$

this in turn implies Proposition 6.1 for $n=3$. Now, since $\operatorname{lk}\left(\Sigma_{1} \cap \Sigma_{2}, K_{3}\right)$ does not vary under a surgery on a knot that does not link $K_{1}, K_{2}$ and $K_{3}$ algebraically, Proposition 6.1 is also true for $n \geq 4$ and hence for all $n$. 
Proof of Proposition 6.3 Use that $\lambda^{\prime}\left(K^{s}\right)=\lambda^{\prime}\left(U, K^{-}\right)$where $U$ is a trivial knot that surrounds the crossing change. (See the proofs of Propositions 5.3 and 12.2 in Section 12.)

\section{Proofs of the statements on $\lambda_{2}$ and $w_{3}$}

Theorem 5.1 guarantees the existence of a polynomial surgery formula $\lambda_{2}(M(K ; p / q))-\lambda_{2}(M)$

$$
=\lambda_{2}^{\prime \prime}(K)(q / p)^{2}+w_{3}(K)(q / p)+C(K ; q / p)+\lambda_{2}(L(p,-q))
$$

where $C(K ; q / p)$ only depends on $q / p \bmod \mathbb{Z}$ and $C(U ; q / p)=0$. As $Z_{2}^{c}(-M)=$ $Z_{2}^{c}(M), w_{3}(K \subset M)=-w_{3}(K \subset(-M))$.

Furthermore, according to Proposition 12.2, if $K^{s}$ is a singular link with two unlinked double points, then $w_{3}\left(K^{s}\right)=-\ell_{12}\left(K^{s}\right) / 2$ and $C\left(K^{s} ; q / p\right)=0$.

The only unproved assertion of Theorem 7.1 is that the knot invariants $C(K ; q / p)$ read $c(q / p) \lambda^{\prime}(K)$ for knots that bound a surface whose $H_{1}$ vanishes in $H_{1}(M)$. The proof of this assertion will be given in this section.

Also note that for any knot $K$ in a rational homology sphere $M, w_{3}(K \subset M)=$ $w_{3}(K \subset M \sharp N)$ and $C(K \subset M ; q / p)=C(K \subset M \sharp N ; q / p)$.

Let $K^{s}$ be a singular knot with one double point in a rational homology sphere. Let $K^{+}$ and $K^{-}$be its two desingularisations, and let $K^{\prime}$ and $K^{\prime \prime}$ be the two knots obtained from $K^{s}$ by smoothing the double point. Assume that $K^{\prime}$ and $K^{\prime \prime}$ are null-homologous, set

$$
f\left(K^{s}\right)=\frac{\lambda^{\prime}\left(K^{\prime}\right)+\lambda^{\prime}\left(K^{\prime \prime}\right)}{2}-\frac{\lambda^{\prime}\left(K^{+}\right)+\lambda^{\prime}\left(K^{-}\right)+\operatorname{lk}^{2}\left(K^{\prime}, K^{\prime \prime}\right)}{4} .
$$

Note that $f\left(K^{s} \subset M\right)=f\left(K^{s} \subset M \sharp N\right)$.

In order to prove Proposition 7.2, we shall successively prove the following lemmas. The two last ones Lemma 14.3 and Lemma 14.4 obviously imply Proposition 7.2.

Lemma 14.1 Let $K^{s}$ be a singular knot with one double point in a rational homology sphere. The invariants $C\left(K^{s} ; q / p\right)$ and $\left(w_{3}-f\right)\left(K^{s}\right)$ do not vary under a surgery on a knot that is null-homologous in the complement of $K^{s}$.

Lemma 14.2 Let $\Gamma$ be a (not necessarily connected) graph in a rational homology sphere $M$, such that every loop of $\Gamma$ is null-homologous in $M$. Then there exist a graph $\Gamma_{0}$ in $S^{3}$, an algebraically split (rationally) framed link $L$ in $S^{3}$ whose components are null-homologous in $S^{3} \backslash \Gamma_{0}$, and a rational homology sphere $N$, such that $\left(S^{3}(L), \Gamma_{0}\right)=(M, \Gamma) \sharp N$. 
Lemma 14.3 Let $K_{n}^{s}$ be the singular knot of Figure 31 where $2 n$ represents $|n|$
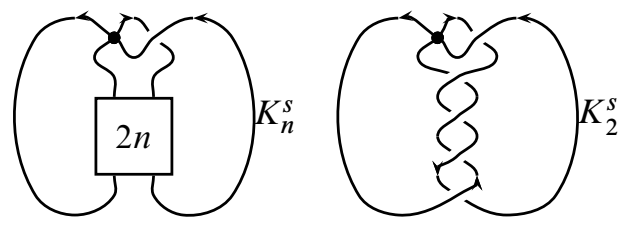

Figure 31

vertical juxtapositions of the pattern ל⿳八人 if $n>0$ and $|n|$ vertical juxtapositions of the pattern $\succ$ if $n<0$. Then for any singular knot $K^{s}$ with one double point $p$, such that the two knots $K^{\prime}$ and $K^{\prime \prime}$ obtained from $K^{s}$ by smoothing $p$ are null-homologous,

$$
\left(w_{3}-f\right)\left(K^{s}\right)=\left(w_{3}-f\right)\left(K_{-\operatorname{lk}\left(K^{\prime}, K^{\prime \prime}\right)}^{s}\right) .
$$

Lemma 14.4 For all $n \in \mathbb{Z},\left(w_{3}-f\right)\left(K_{n}^{s}\right)=0$.

We shall next prove the following proposition that generalizes a Casson lemma from integral to rational homology spheres.

Proposition 14.5 Let $C$ be a real-valued invariant of null-homologous knots in rational homology spheres such that

- $C(K \subset M)=C(K \subset M \sharp N)$,

- $C(U)=0$,

- $C(K)$ does not vary under a surgery on a knot $J$ such that $(J, K)$ is a boundary link,

- if $K^{s}$ is a singular knot with one double point, $C\left(K^{s}\right)$ does not vary under surgery on a knot that is null-homologous in the complement of $K^{s}$.

Then there exists $c \in \mathbb{R}$ such that

- if $K^{s}$ is a singular knot with one double point $p$, such that the two knots $K^{\prime}$ and $K^{\prime \prime}$ obtained from $K^{s}$ by smoothing $p$ are null-homologous, then $C\left(K^{s}\right)=c \operatorname{lk}\left(K^{\prime}, K^{\prime \prime}\right)$,

- if $K$ bounds a surface whose $H_{1}$ maps to zero in $H_{1}(M), C(K)=c \lambda^{\prime}(K)$.

Since the $C(. ; p / q)$ satisfy the hypotheses of the proposition above (thanks to Theorem 4.1 for the hypothesis on boundary links), this proposition will be sufficient to conclude the proof of Theorem 7.1.

Let us now prove all the lemmas and the proposition. 
Proof of Lemma 14.1 Let $J$ be a null-homologous knot unlinked with $K^{\prime}$ and $K$. Let $F_{J}$ be a Seifert surface for $J$ that does not meet $K^{s}$, and let $(m, \ell)$ be the usual basis of the genus one surface obtained by tubing a trivial knot $V$ surrounding the double point of $K^{s}, m$ is a meridian of $K^{-}, \ell$ is homotopic to $K^{\prime}$ and $\operatorname{lk}\left(\ell, K^{-}\right)=0$. By Theorem 4.1,

$$
\begin{aligned}
Z_{2}^{c}\left(M\left(J ; \frac{p_{J}}{q_{J}}\right)\left(K^{s} ; \frac{p}{q}\right)\right)-Z_{2}^{c} & \left(M\left(K^{s} ; \frac{p}{q}\right)\right) \\
& =\frac{q_{J}}{4 p_{J}} p^{c}\left(\|\left(\begin{array}{ll}
\ell m^{+} \ell^{+} \\
\vee
\end{array}\left(F_{J}\right) \subset M\left(K^{-} ; \frac{p}{q}\right) \|\right) .\right.
\end{aligned}
$$

Since, according to Lemma 9.5, the pairing of $m$ and a curve $c$ in the contraction above will give rise to the coefficient $(-q / p) \operatorname{lk}(K, c)=-r \operatorname{lk}(K, c), C\left(K^{s} ; q / p\right)$ does not vary under a $\left(p_{J} / q_{J}\right)$-surgery on $J$.

$$
\begin{aligned}
w_{3}\left(K^{s} \subset M\left(J ; \frac{p_{J}}{q_{J}}\right)\right) & -w_{3}\left(K^{s} \subset M\right) \\
= & \left(\frac{\partial}{\partial r}\right)_{r=0} W_{2}\left(Z_{2}^{c}\left(K^{s} \subset M\left(J ; \frac{p_{J}}{q_{J}}\right)\right)-Z_{2}^{c}\left(K^{s} \subset M\right)\right)
\end{aligned}
$$

where $m$ must be paired either with $m^{+}$or with $I\left(F_{J}\right)$, and in the latter case $m^{+}$ must be paired with $\ell$ in order to lead to a linear contribution in $r$.

$$
\begin{aligned}
& w_{3}\left(K^{s} \subset M\left(J ; \frac{p_{J}}{q_{J}}\right)\right)-w_{3}\left(K^{s} \subset M\right)=
\end{aligned}
$$

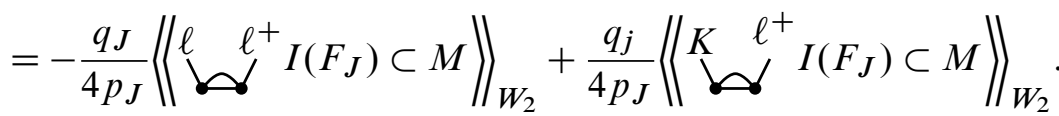

Since $K=K^{\prime \prime}+\ell$, as far as the connected pairing with $I\left(F_{J}\right)$ is concerned,

$$
K-\infty-K^{+}=\ell-\infty-\ell^{+}+K^{\prime \prime}-\infty-K^{\prime \prime+}+2 \ell-\infty-K^{\prime \prime}
$$

and

$$
K-\infty-\ell^{+}=\ell-\infty-\ell^{+}+K^{\prime \prime}-\infty-\ell^{+} \text {. }
$$

Therefore,

$$
K \rightarrow \infty-\ell^{+}=\frac{1}{2} \ell-\infty-\ell^{+}-\frac{1}{2} K^{\prime \prime}-\infty-K^{\prime \prime+}+\frac{1}{2} K \rightarrow \infty-K^{+} .
$$

$w_{3}\left(K^{s} \subset M\left(J ; \frac{p_{J}}{q_{J}}\right)\right)-w_{3}\left(K^{s} \subset M\right)$

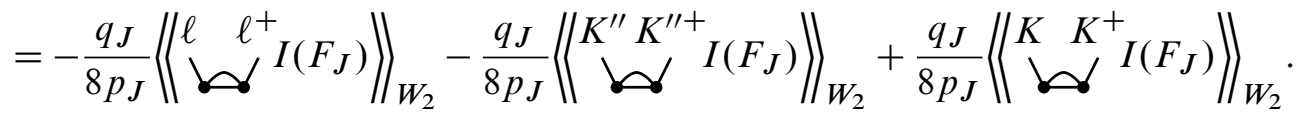


Thus, according to Proposition 6.2, since

$$
\begin{aligned}
& \left.\left\langle K-\infty-K^{+} I\left(F_{J}\right)\right\rangle\right\rangle_{W_{2}}=\left\langle\left\langle K-K^{+} I\left(F_{J}\right)\right\rangle\right\rangle_{W_{1}}, \\
w_{3}\left(K^{s} \subset\right. & \left.M\left(J ; \frac{p_{J}}{q_{J}}\right)\right)-w_{3}\left(K^{s} \subset M\right) \\
= & \frac{q_{J}}{2 p_{J}}\left(\lambda^{\prime}\left(J, K^{\prime}\right)+\lambda^{\prime}\left(J, K^{\prime \prime}\right)-\lambda^{\prime}(J, K)\right) \\
= & \frac{q_{J}}{p_{J}}\left(\frac{\lambda^{\prime}\left(J, K^{\prime}\right)}{2}+\frac{\lambda^{\prime}\left(J, K^{\prime \prime}\right)}{2}-\frac{\lambda^{\prime}\left(J, K^{+}\right)}{4}-\frac{\lambda^{\prime}\left(J, K^{-}\right)}{4}\right) \\
= & f\left(K^{s} \subset M\left(J ; \frac{p_{J}}{q_{J}}\right)\right)-f\left(K^{s} \subset M\right) .
\end{aligned}
$$

Proof of Lemma 14.2 After a possible connected sum with some lens spaces, the $\mathbb{Q} / \mathbb{Z}$-valued linking form of $M$ is diagonal (see Wall [36]), and the generators of $H_{1}(M ; \mathbb{Z})$ can be represented by a link $L$ of algebraically unlinked curves $K_{i}$ that do not link $\Gamma$, algebraically. Then for each $K_{i}$, there exists a surface $\Sigma_{i}$ in the exterior ( $M \backslash \operatorname{Int} N(L))$ of $L$ whose boundary is a connected essential curve of $\partial N\left(K_{i}\right)$, and that does not meet $\Gamma$. Thus, $H^{1}(M \backslash \operatorname{Int} N(L) ; \mathbb{Z})$ is freely generated by the algebraic intersections with the $\Sigma_{i}$, and there exists a surgery on $L$ that transforms $M$ into a homology sphere $H$. The manifold $H$ can in turn be transformed into $S^{3}$ by surgery on a boundary link of $H$ bounding a disjoint union $F_{H}$ of surfaces in $H$ that can be assumed to be disjoint from the first surgery link and from the image of $\Gamma$ in $H$. This proves the lemma.

Proof of Lemma 14.3 Apply Lemma 14.2 to $\Gamma=K^{s}$, then $K^{s, 0}=\Gamma_{0}$. Note that $\operatorname{lk}\left(K_{0}^{\prime}, K_{0}^{\prime \prime}\right)=\operatorname{lk}\left(K^{\prime}, K^{\prime \prime}\right)$. Recall that $\left(w_{3}-f\right)\left(K^{s} \subset M\right)=\left(w_{3}-f\right)\left(K^{s} \subset M \sharp N\right)$. Thanks to Lemma 14.1, $\left(w_{3}-f\right)\left(K^{s} \subset M \sharp N\right)=\left(w_{3}-f\right)\left(K^{s, 0} \subset S^{3}\right)$. Now that the proof has been reduced to the case where $M=S^{3}$, recall that a crossing change on $K^{\prime}$ or $K^{\prime \prime}$ may be realized by a surgery on a knot satisfying the hypotheses of Lemma 14.1, that changes neither $\operatorname{lk}\left(K^{\prime}, K^{\prime \prime}\right)$ nor $\left(w_{3}-f\right)\left(K^{s}\right)$. Unknotting $K^{\prime}$ first by crossing changes and next unknotting the parts of $K^{\prime \prime}$ between two consecutive intersection points with the disk bounded by $K^{\prime}$ transforms $K^{s}$ into $K_{-1 \mathrm{k}\left(K^{\prime}, K^{\prime \prime}\right)}^{s} \cdot \square$

Proof of Lemma 14.4 By the crossing change formula of Proposition 6.3, $\lambda^{\prime}\left(K_{n}^{+}\right)-$ $\lambda^{\prime}\left(K_{n-1}^{+}\right)=-1$, and $\lambda^{\prime}\left(K_{n}^{+}\right)=-n$. Since $K_{n}^{-}, K_{n}^{\prime}$ and $K_{n}^{\prime \prime}$ are trivial, $f\left(K_{n}^{s}\right)=$ $-n(n-1) / 4$.

On the other hand, since $w_{3}\left(K_{n}^{-}\right)=0, w_{3}\left(K_{n}^{s}\right)=w_{3}\left(K_{n}^{+}\right)$. The unlinked double crossing change formula of Theorem 7.1 implies that

$$
w_{3}\left(K_{n+2}^{+}\right)-2 w_{3}\left(K_{n+1}^{+}\right)+w_{3}\left(K_{n}^{+}\right)=-1 / 2 .
$$


Since $K_{0}^{+}$is trivial, $w_{3}\left(K_{0}^{+}\right)=0$, and since $K_{1}^{+}$is the figure-eight knot that coincides with its mirror image, $w_{3}\left(K_{1}^{+}\right)=0$, too. Then $w_{3}\left(K_{n}^{s}\right)=w_{3}\left(K_{n}^{+}\right)=-n(n-1) / 4$.

Proof of Proposition 14.5 Let $K^{s}$ be as in the hypotheses of Proposition 7.2. The proof of Lemma 14.3 shows that $C\left(K^{s}\right)=C\left(K_{-1 \mathrm{k}\left(K^{\prime}, K^{\prime \prime}\right)}^{s}\right)$. Since $C(U)=0$, $C\left(K_{n}^{s}\right)=C\left(K_{n}^{+}\right)$. Since the hypotheses of the proposition imply that $C$ maps singular knots of $S^{3}$ with two unlinked double points to $0, C\left(K_{n+2}^{+}\right)-2 C\left(K_{n+1}^{+}\right)+C\left(K_{n}^{+}\right)=0$, and $C\left(K_{n}^{+}\right)$is affine with respect to $n$. Since $C\left(K_{0}^{+}\right)=0, C\left(K_{n}^{+}\right)$is linear. Then there exists $c$ such that $C\left(K^{s}\right)=c \operatorname{lk}\left(K^{\prime}, K^{\prime \prime}\right)$.

Let $K$ be a knot that bounds a Seifert surface $\Sigma$ whose $H_{1}$ maps to zero in $H_{1}(M)$. Applying Lemma 14.2 to the one-skeleton of $\Sigma$ allows us to reduce the proof that $C(K)=c \lambda^{\prime}(K)$ to the case of knots in $S^{3}$, thanks to the hypothesis on boundary links. Then this case is easily proved with the crossing change formula.

Proof of Proposition 7.3 Consider the genus one surface $\Sigma$ in $H$ and its symplectic basis $(a, b)$ shown in Figure 32. Here, $\langle a, b\rangle=1, \operatorname{lk}\left(a, a^{+}\right)=\frac{x+z}{2}, \operatorname{lk}\left(b, b^{+}\right)=\frac{y+z}{2}$,

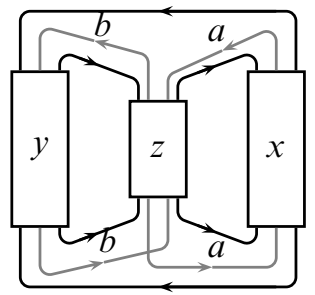

Figure 32

$\operatorname{lk}\left(a, b^{+}\right)=\frac{-1-z}{2}, \operatorname{lk}\left(a^{+}, b\right)=\frac{1-z}{2}$ and

$$
\lambda^{\prime}(K(x, y, z))=\frac{(x+z)(y+z)+1-z^{2}}{4}=\frac{x y+y z+z x+1}{4} .
$$

Note that $\lambda^{\prime}(\phi(X), \phi(Y))=\lambda^{\prime}(\phi(Y), \phi(Z))=\lambda^{\prime}(\phi(Z), \phi(X))$. In particular, both sides of the equality which we are proving are symmetric under a cyclic permutation of $((X, x),(Y, y),(Z, z))$. Using this cyclic symmetry, the formula for the pretzel knot $K(x, y, z)$ follows from the crossing change formula starting with the trivial knot $K_{-1,1,1}$ :

$$
\begin{aligned}
4 w_{3}(K(x+2, y, z))-4 w_{3}(K(x, y, z)) & \\
& =\lambda^{\prime}(K(x+2, y, z))+\lambda^{\prime}(K(x, y, z))+\left(\frac{y+z}{2}\right)^{2},
\end{aligned}
$$




$$
\begin{gathered}
16\left(w_{3}(K(x+2, y, z))-w_{3}(K(x, y, z))\right)=(2 x+2)(y+z)+2+4 y z+y^{2}+z^{2}, \\
32 w_{3}(K(x, y, z))=2 x+4 x y z+x y^{2}+x z^{2}+x^{2}(y+z)+F(y, z) .
\end{gathered}
$$

Otherwise, the following Lemma 14.6 reduces the proof of Proposition 7.3 to the case where the knot $\phi(K(x, y, z))$ is in $S^{3}$, thanks to Lemma 14.2, and next when the knot is a pretzel knot $K(x, y, z)$ by crossing changes on $X$ and $Y$.

Lemma 14.6 Let $\phi$ be an embedding of $H$ in a rational homology sphere such that $\phi(X)$ and $\phi(Y)$ are null-homologous in the exterior of $\phi(H)$. Let $J$ be a knot in the exterior of $\phi(H)$ that links neither $\phi(X)$ nor $\phi(Y)$, then

$$
\begin{aligned}
& w_{3}(\phi(K(x, y, z)) \subset M(J ; p / q))-w_{3}(\phi(K(x, y, z)) \subset M) \\
& \quad=\frac{q}{2 p}\left(3 \lambda^{\prime}(\phi(X), \phi(Y), J)-x \lambda^{\prime}(\phi(X), J)-y \lambda^{\prime}(\phi(Y), J)-z \lambda^{\prime}(\phi(Z), J)\right) .
\end{aligned}
$$

Proof of Lemma 14.6 According to Theorem 4.1, if $F_{J}$ is a Seifert surface of $J$ in the complement of the genus one Seifert surface $\Sigma$ of $\phi(K(x, y, z))$ in $\phi(H)$,

$w_{3}(\phi(K(x, y, z)) \subset M(J ; p / q))-w_{3}(\phi(K(x, y, z)) \subset M)=\frac{q}{4 p}\left\langle\left\langle I(\Sigma) I\left(F_{J}\right)\right\rangle\right\rangle_{W_{2}}$

where

$$
I(\Sigma)=\stackrel{a}{a} b^{+} b^{+}
$$

Write

$$
\frac{q}{4 p}\left\langle\left\langle I(\Sigma) I\left(F_{J}\right)\right\rangle\right\rangle_{W_{2}}=C_{A}+C_{B}
$$

where $C_{A}$ is the contribution of the pairings that pair two univalent vertices of $I(\Sigma)$, and $C_{B}$ is the contribution of the pairings that pair all the univalent vertices of $I(\Sigma)$ to univalent vertices of $I\left(F_{J}\right)$.

$$
C_{A}=\frac{q}{4 p} \|\left\langle\left(\frac{x+z}{2} b-\infty-b^{+}+\frac{y+z}{2} a-\infty-a^{+}+z a-\infty-b\right) I\left(F_{J}\right) \|_{W_{2}}\right.
$$

From now on, we write $X, Y$ and $Z$ for $\phi(X), \phi(Y)$ and $\phi(Z)$, respectively.

$$
C_{A}=\frac{q}{4 p} \|\left\langle\left(\frac{x}{2} X-\infty-X^{+}+\frac{y}{2} Y \rightarrow \infty-Y^{+}+\frac{z}{2}(X+Y)-\infty-(X+Y)^{+}\right) I\left(F_{J}\right) \|_{W_{2}}\right.
$$

Thus, according to Proposition 6.2,

$$
C_{A}=-\frac{q}{p}\left(\frac{x \lambda^{\prime}(X, J)}{2}+\frac{y \lambda^{\prime}(Y, J)}{2}+\frac{z \lambda^{\prime}(Z, J)}{2}\right) .
$$

Let us now compute the contribution of the pairings that are bijections from the set of univalent vertices of $I(\Sigma)$ to the set of univalent vertices of $I\left(F_{j}\right)$. For them, we may 
change $a$ to $Y$ and $b$ to $X$ and write

$$
I(\Sigma)=\stackrel{X Y Y^{+} X^{+}}{\vee}
$$

where the superscripts + distinguish two copies of $X$ (or $Y$ ) whose linking numbers with the curves of $F_{J}$ are the same.

Let us compute the contribution $C_{B}$ of the pairings that are bijections from the set of univalent vertices of $I(\Sigma)$ to the set of univalent vertices of some

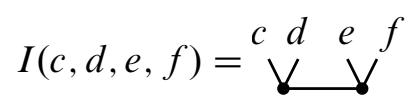

to $\langle\langle I(c, d, e, f) I(\Sigma)\rangle\rangle_{W_{2}}$.

Note the symmetry under the exchange of the pair $(X, Y)$ with the pair $\left(X^{+}, Y^{+}\right)$.

The contribution of the pairings that pair $c$ and $d$ to $X$ and $X^{+}$is

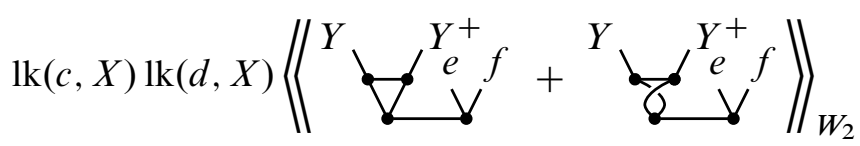

that is zero by the antisymmetry relation in the space of Jacobi diagrams. Similarly, the contribution of the pairings that pair $c$ and $d$ to $Y$ and $Y^{+}$vanishes.

The contributions of the pairings that pair $d$ and $e$ to $X$ and $X^{+}$is

$$
2 \operatorname{lk}(d, X) \operatorname{lk}(e, X) \operatorname{lk}(c, Y) \operatorname{lk}(f, Y) W_{2}(88+\triangle)
$$

where

$$
W_{2}(\triangle)=W_{2}(\triangle)=1 \text {. }
$$

Therefore, the contribution to $\langle\langle I(c, d, e, f) I(\Sigma)\rangle\rangle_{W_{2}}$ of the pairings that are bijections from the set of univalent vertices of $I(\Sigma)$ to the set of univalent vertices of $I(c, d, e, f)$ is

$$
\frac{3}{4}\left\langle\left\langle I(c, d, e, f) X-X^{+} \quad Y-Y^{+}\right\rangle\right\rangle_{W_{1}} .
$$

Therefore, according to Proposition 6.2,

$$
\begin{aligned}
C_{B} & =\frac{q}{4 p} \frac{3}{4}\left\langle\left\langle I\left(F_{J}\right) X-X^{+} \quad Y-Y^{+}\right\rangle_{W_{1}}=3 \frac{q}{2 p} \lambda^{\prime}(J, X, Y)\right. \\
& \left.=\frac{3}{2}\left(\lambda^{\prime}((X, Y) \subset M(J ; q / p))-\lambda^{\prime}((X, Y) \subset M)\right)\right) .
\end{aligned}
$$

Proposition 7.3 is proved. 


\section{More about surgeries on general knots in rational homol- ogy spheres}

Theorem 5.1 describes the polynomial behaviour of $Z_{n}$ under surgeries on null-homologous knots. It can easily be generalized to the case of non-null-homologous knots $K$ a primitive satellite $\ell$ of which bounds a Seifert surface. Let $m_{K}$ be the meridian of such a knot $K$ such that $\left\langle m_{K}, \ell\right\rangle_{\partial N(K)}=O_{K}$.

A surgery curve $\mu$ on $\partial N(K)$ is determined by its coordinates $\left(p_{K}, q_{K}\right)$ in the symplectic basis $\left(m_{K}, \frac{1}{O_{K}} \ell\right)$ of $H_{1}(\partial N(K) ; \mathbb{Q})$ where $p_{K}=\frac{1}{O_{K}}\langle\mu, \ell\rangle$ is the linking number of $K$ and $\mu$, and $q_{K}=\left\langle m_{K}, \mu\right\rangle$. The associate surgery coefficient is $p_{K} / q_{K}$.

Theorem 15.1 Let $n \in \mathbb{N}$. Let $K$ be a knot of order $O_{K}$ in a rational homology sphere $M$ such that a primitive satellite $\ell$ of $K$ bounds a Seifert surface $F$. Let $F^{1}, \ldots, F^{n}$ be parallel copies of $F$. Let $p_{K} / q_{K} \in \mathbb{Q}$ be a surgery coefficient for $K$. Then

$$
Z_{n}\left(M\left(K ; \frac{p_{K}}{q_{K}}\right)\right)-Z_{n}(M)=\sum_{i=0}^{n} Y_{n, q_{K} /\left(p_{K} O_{K}^{2}\right)}^{(i)}(K \subset M)\left(\frac{q_{K}}{p_{K}}\right)^{i}
$$

where

$$
\left.\left.Y_{n, q_{K} /\left(p_{K} O_{K}^{2}\right)}^{(n)}(K)=\frac{1}{n ! 2^{n} O_{K}^{2 n}} \| \bigsqcup_{i \in\{1, \ldots, n\}} I\left(F^{i}\right)\right)\right\rangle
$$

$Y_{n, q_{K} /\left(p_{K} O_{K}^{2}\right)}^{(i)}$ only depends on $q_{K} /\left(p_{K} O_{K}^{2}\right) \bmod \mathbb{Z}$, and, if $n \geq 2$,

$$
p_{c}\left(Y_{n, q_{K} /\left(p_{K} O_{K}^{2}\right)}^{(n-1)}\right)=Y_{n, q_{K} /\left(p_{K} O_{K}^{2}\right)}^{(n-1) c}
$$

does not depend on $p_{K}$ and $q_{K}$. Furthermore, if $m$ is a primitive satellite of $K$ such that $\langle m, \ell\rangle_{\partial N(K)}=1$, and if $\widehat{K} \subset \widehat{M}$ is the knot with the same complement as $K$ whose meridian is $m$, then, if $n \geq 2$,

$$
Y_{n}^{(n-1) c}(K \subset M)=\frac{1}{O_{K}^{2 n-2}} Y_{n}^{(n-1) c}(\widehat{K} \subset \widehat{M})+n\left\langle m, m_{K}\right\rangle O_{K} Y_{n}^{(n) c}(K \subset M) \text {. }
$$

Proof Let $\mu=p_{K} m_{K}+\left(q_{K} / O_{K}\right) \ell$ be a surgery curve on $\partial N(K)$. Let

$$
\left(p=\langle\mu, \ell\rangle=O_{K} p_{K}, q=\langle m, \mu\rangle=p_{K}\left\langle m, m_{K}\right\rangle+q_{K} / O_{K}\right)
$$

be the coordinates of $\mu$ in the symplectic basis $(m, \ell)$ of $H_{1}(N(K) ; \mathbb{Z})$. Note that changing $m$ to another curve such that $\langle m, \ell\rangle_{\partial N(K)}=1$ leaves $p$ invariant and does not change the class of $\frac{q}{p}$ in $\mathbb{Q} / \mathbb{Z}$. When the other data are fixed, the mod $\mathbb{Z}$ congruence class of

$$
\frac{q}{p}=\frac{q_{K}}{p_{K} O_{K}^{2}}+\frac{\left\langle m, m_{K}\right\rangle}{O_{K}}
$$


depends on the class of $q_{K} /\left(p_{K} O_{K}^{2}\right)$ in $\mathbb{Q} / \mathbb{Z}$. From the formula of Theorem 5.1

$$
Z_{n}\left(\widehat{M}\left(\widehat{K} ; \frac{p}{q+r p}\right)\right)-Z_{n}(\widehat{M})=\sum_{i=0}^{n} Y_{n, q / p}^{(i)}(\widehat{K} \subset \widehat{M})\left(r+\frac{q}{p}\right)^{i},
$$

we deduce

$$
\begin{aligned}
Z_{n}( & \left.M\left(K ; \frac{p_{K}}{q_{K}+r O_{K}^{2} p_{K}}\right)\right)-Z_{n}(M) \\
= & \sum_{i=0}^{n} Y_{n, q / p}^{(i)}(\widehat{K} \subset \widehat{M})\left(r+\frac{q_{K}}{p_{K} O_{K}^{2}}+\frac{\left\langle m, m_{K}\right\rangle}{O_{K}}\right)^{i}+Z_{n}(\widehat{M})-Z_{n}(M) \\
= & \sum_{i=0}^{n} Y_{n, q_{K} /\left(p_{K} O_{K}^{2}\right)}^{(i)}(K \subset M)\left(r O_{K}^{2}+\frac{q_{K}}{p_{K}}\right)^{i}
\end{aligned}
$$

where

$$
\left.\left.Y_{n, q_{K} /\left(p_{K} O_{K}^{2}\right)}^{(n)}(K \subset M)=\frac{1}{n ! 2^{n} O_{K}^{2 n}} \| \bigsqcup_{i \in\{1, \ldots, n\}} I\left(F^{i}\right)\right\rangle\right)
$$

and, if $n \geq 2$,

$$
\begin{aligned}
Y_{n, q_{K} /\left(p_{K} O_{K}^{2}\right)}^{(n-1)} & (K \subset M) \\
= & \frac{1}{O_{K}^{2 n-2}} Y_{n, q / p}^{(n-1)}(\widehat{K} \subset \widehat{M})+n\left\langle m, m_{K}\right\rangle O_{K} Y_{n}^{(n)}(K \subset M) .
\end{aligned}
$$

Remarks 15.2 A knot $K$ of order $O_{K}$ in a rational homology sphere has a primitive satellite that is null-homologous in its exterior if and only if the self-linking number of $K$ reads $d / O_{K}(\bmod \mathbb{Z})$ where $d$ is coprime with $O_{K}$.

Like in the proof of Theorem 15.1, the case of knots without null-homologous primitive satellites can be reduced to the case of knots of order $O_{K}>1$ with self-linking number 0 . This latter case is still unclear to me (except for the degree 1 case that can be treated with the methods of the article).

Relationships between surgery formulae for various $q / p$ can be found using some equivalences of surgeries. See Garoufalidis and Ohtsuki [16].

\section{Questions}

The statements of Theorem 4.3 and Theorem 4.4 make sense for rationally algebraically split links. Do they hold true in this case? 
How do the properties of surgery formulae generalize for surgeries on knots which are not null-homologous?

What is the graded space associated with the filtration of the rational vector space generated by rational homology spheres, defined using Lagrangian-preserving surgeries?

The degree $n$ parts of the LMO invariant and the Kontsevich-Kuperberg-Thurston configuration space invariant coincide on the intersection of $\mathcal{F}_{n}$ with the vector space generated by homology spheres. The configuration space invariant for knots in $S^{3}$ is obtained from the Kontsevich integral by an isomorphism that inserts a (possibly trivial) specific two-leg box $\beta$ on each chord of a chord diagram. See the author's paper [28] for a more specific statement. Do the LMO invariant and the Kontsevich-KuperbergThurston invariant actually coincide? Is the Kontsevich-Kuperberg-Thurston invariant obtained from the LMO invariant by inserting the two-leg box $\beta, k$ (or $2 k$ or $3 k$ ) times on each degree $k$ component of a Jacobi diagram?

\section{References}

[1] S Akbulut, J D McCarthy, Casson's invariant for oriented homology 3-spheres. An exposition, Math. Notes 36, Princeton Univ. Press (1990) MR1030042

[2] E Auclair, Surfaces et invariants de type fini en dimension 3, PhD thesis, Grenoble University (2006)

[3] E Auclair, C Lescop, Clover calculus for homology 3-spheres via basic algebraic topology, Algebr. Geom. Topol. 5 (2005) 71-106 MR2135546

[4] S Axelrod, I M Singer, Chern-Simons perturbation theory, from: "Proceedings of the XXth International Conference on Differential Geometric Methods in Theoretical Physics, Vol. 1, 2 (New York, 1991)", (S Catto, A Rocha, editors), World Sci. Publ., River Edge, NJ (1992) 3-45 MR1225107

[5] S Axelrod, I M Singer, Chern-Simons perturbation theory. II, J. Differential Geom. 39 (1994) 173-213 MR1258919

[6] D Bar-Natan, On the Vassiliev knot invariants, Topology 34 (1995) 423-472 MR1318886

[7] D Bar-Natan, S Garoufalidis, L Rozansky, D P Thurston, The Århus integral of rational homology 3-spheres. I. A highly non trivial flat connection on $S^{3}$, Selecta Math. (N.S.) 8 (2002) 315-339 MR1931167

[8] D Bar-Natan, S Garoufalidis, L Rozansky, D P Thurston, The Århus integral of rational homology 3-spheres. II. Invariance and universality, Selecta Math. (N.S.) 8 (2002) 341-371 MR1931168 
[9] D Bar-Natan, S Garoufalidis, L Rozansky, D P Thurston, The Arhus integral of rational homology 3-spheres. III. Relation with the Le-Murakami-Ohtsuki invariant, Selecta Math. (N.S.) 10 (2004) 305-324 MR2099069

[10] R Bott, A S Cattaneo, Integral invariants of 3-manifolds, J. Differential Geom. 48 (1998) 91-133 MR1622602

[11] R Bott, A S Cattaneo, Integral invariants of 3-manifolds. II, J. Differential Geom. 53 (1999) 1-13 MR1776090

[12] A S Cattaneo, Configuration space integrals and invariants for 3-manifolds and knots, from: "Low-dimensional topology (Funchal, 1998)", (H Nencka, editor), Contemp. Math. 233, Amer. Math. Soc. (1999) 153-165 MR1701680

[13] S Garoufalidis, On finite type 3-manifold invariants. I, J. Knot Theory Ramifications 5 (1996) 441-461 MR1406715

[14] S Garoufalidis, M Goussarov, M Polyak, Calculus of clovers and finite type invariants of 3-manifolds, Geom. Topol. 5 (2001) 75-108 MR1812435

[15] S Garoufalidis, N Habegger, The Alexander polynomial and finite type 3-manifold invariants, Math. Ann. 316 (2000) 485-497 MR1752781

[16] S Garoufalidis, T Ohtsuki, On finite type 3-manifold invariants. V. Rational homology 3-spheres, from: "Geometry and physics (Aarhus, 1995)", (J E Andersen, J Dupont, H Pedersen, A Swann, editors), Lecture Notes in Pure and Appl. Math. 184, Dekker, New York (1997) 445-457 MR1423186

[17] C M Gordon, Some aspects of classical knot theory, from: "Knot theory (Proc. Sem., Plans-sur-Bex, 1977)”, (J-C Hausmann, editor), Lecture Notes in Math. 685, Springer, Berlin (1978) 1-60 MR521730

[18] L Guillou, A Marin, Notes sur l'invariant de Casson des sphères d'homologie de dimension trois, Enseign. Math. (2) 38 (1992) 233-290 MR1189008 With an appendix by C Lescop

[19] K Habiro, Claspers and finite type invariants of links, Geom. Topol. 4 (2000) 1-83 MR1735632

[20] C Kearton, W B R Lickorish, Piecewise linear critical levels and collapsing, Trans. Amer. Math. Soc. 170 (1972) 415-424 MR0310899

[21] M Kontsevich, Feynman diagrams and low-dimensional topology, from: "First European Congress of Mathematics, Vol. II (Paris, 1992)”, (A Joseph, F Mignot, F Murat, B Prum, R Rentschler, editors), Progr. Math. 120, Birkhäuser, Basel (1994) 97-121 MR1341841

[22] G Kuperberg, D P Thurston, Perturbative 3-manifold invariants by cut-and-paste topology arXiv:math.GT/9912167 
[23] T T Q Le, An invariant of integral homology 3-spheres which is universal for all finite type invariants, from: "Solitons, geometry, and topology: on the crossroad", (V M Buchstaber, S P Novikov, editors), Amer. Math. Soc. Transl. Ser. 2 179, Amer. Math. Soc. (1997) 75-100 MR1437158

[24] T T Q Le, J Murakami, T Ohtsuki, On a universal perturbative invariant of 3manifolds, Topology 37 (1998) 539-574 MR1604883

[25] C Lescop, On the Kontsevich-Kuperberg-Thurston construction of a configuration space invariant for rational homology 3-spheres arXiv:math.GT/0411088

[26] C Lescop, Splitting formulae for the Kontsevich-Kuperberg-Thurston invariant of rational homology 3-spheres arXiv:math.GT/0411431

[27] C Lescop, Global surgery formula for the Casson-Walker invariant, Annals of Math. Studies 140, Princeton Univ. Press (1996) MR1372947

[28] C Lescop, About the uniqueness of the Kontsevich integral, J. Knot Theory Ramifications 11 (2002) 759-780 MR1918812

[29] A Marin, Un nouvel invariant pour les sphères d'homologie de dimension trois (d'après Casson), from: “Séminaire Bourbaki, Vol. 1987/88, Exp. No. 693”, Astérisque 161-162 (1988) 151-164 MR992207

[30] S V Matveev, Generalized surgeries of three-dimensional manifolds and representations of homology spheres, Mat. Zametki 42 (1987) 268-278, 345 MR915115 In Russian; translated in Math. Notes 42 (1999) 651-656

[31] J Milnor, Link groups, Ann. of Math. (2) 59 (1954) 177-195 MR0071020

[32] H Murakami, Y Nakanishi, On a certain move generating link-homology, Math. Ann. 284 (1989) 75-89 MR995383

[33] T Ohtsuki, Finite type invariants of integral homology 3-spheres, J. Knot Theory Ramifications 5 (1996) 101-115 MR1373813

[34] H Rademacher, E Grosswald, Dedekind sums, The Carus Math. Monogr. 16, Math. Assoc. Amer., Washington, D.C. (1972) MR0357299

[35] K Walker, An extension of Casson's invariant, Annals of Math. Studies 126, Princeton Univ. Press (1992) MR1154798

[36] C T C Wall, Quadratic forms on finite groups, and related topics, Topology 2 (1963) 281-298 MR0156890

Institut Fourier

BP 74, 38402 Saint-Martin d'Hères cedex, France

lescop@ujf-grenoble.fr

http://www-fourier.ujf-grenoble.fr/ lescop/

Received: 1 April 2009 